TRANSACTIONS OF THE

AMERICAN MATHEMATICAL SOCIETY

Volume 326, Number 1, July 1991

\title{
HYPERBOLIC INVARIANTS OF KNOTS AND LINKS
}

\author{
COLIN ADAMS, MARTIN HILDEBRAND AND JEFFREY WEEKS
}

\begin{abstract}
Tables of values for the hyperbolic volume, number of symmetries, cusp volume and conformal invariants of the cusps are given for hyperbolic knots through ten crossings and hyperbolic links of 2, 3 and 4 components through 9 crossings. The horoball patterns and the canonical triangulations are displayed for knots through eight crossings and for particularly interesting additional examples of knots and links.
\end{abstract}

\section{INTRODUCTION}

In 1978, copies of notes from a course Thurston had taught at Princeton first began to circulate. These notes were the basis for a revolution in 3-manifold theory. Thurston's Geometrization Conjecture, now proven for Haken 3-manifolds and manifolds with symmetry, asserts that the interior of every compact 3manifold may be canonically decomposed into pieces which admit certain geometric structures. (For an overview, see [14].) Of the eight geometries which occur, seven are well understood and the manifolds admitting them have been classified. The last and richest geometry is that of hyperbolic 3-space. Empirically, a "typical" 3-manifold can be expected to admit a hyperbolic structure, with no decomposition required. Mostow's Rigidity Theorem tells us that a finite volume hyperbolic 3-manifold has a unique hyperbolic structure. In particular, the hyperbolic volume can be used as a topological invariant.

In his notes Thurston displays the hyperbolic structure on the figure-eight knot complement as a pair of ideal (vertices missing) hyperbolic tetrahedra with all dihedral angles $\pi / 3$ such that faces are identified in pairs. A similar description for the hyperbolic structure on any hyperbolic knot or link complement in $S^{3}$ is possible.

In this paper we present tables of hyperbolic link invariants. The computer program which calculates the hyperbolic structures decomposes a link complement into ideal tetrahedra according to the algorithm in [15], represents the

Received by the editors September 30, 1988 and, in revised form, May 3, 1989.

1980 Mathematics Subject Classification (1985 Revision). Primary 57M25; Secondary 30F40.

Key words and phrases. Hyperbolic 3-manifold, hyperbolic volume, cusp volume, horoball pattern.

The first author was supported in part by NSF Grant DMS-8711495 and research funds from Williams College. The second author was supported in part by research funds from Williams College and NSF Geometry Supercomputer Project. The third author was supported in part by the NSF Geometry Supercomputer Project and his spouse. 
shape of each ideal tetrahedron by a complex number, sets up the gluing and cusp equations as in [13], and solves the equations using the Newton-Raphson method. From this, the invariants can be calculated.

$\S 2$ briefly describes each of the tabulated invariants, $\S 3$ gives some applications, and the tables themselves appear as Tables 1 and 2. In Table 1, a link denoted Lmnnppp is an $m$-component link with $n n$ crossings and with number $p p p$ in the standard tables for $n n$-crossing links (cf. [11]).

The Dehn surgery spaces for these manifolds have also been calculated (cf. [15]). Recently, C. Hodgson, R. Meyerhoff and W. Neumann have extended the program to also calculate the Chern-Simons invariant for certain manifolds, an invariant which is not discussed in this paper.

A version of the program which generates these results and runs on a Macintosh computer is available from Jeffrey Weeks.

\section{INVARIANTS}

Mostow's Rigidity Theorem (cf. Chapter 5 of [13]) implies that the hyperbolic structure on a finite volume hyperbolic 3-manifold is unique up to isometry. In particular, any invariants which are defined in terms of the hyperbolic structure of such a manifold are topological invariants of the 3-manifold.

The first such invariant that one would like to look at is the hyperbolic volume of the manifold. If the manifold has been decomposed into ideal hyperbolic tetrahedra, the volume will simply be the sum of the volumes of the tetrahedra. Once the dihedral angles on the set of ideal tetrahedra are known, their volumes may be computed using the formula which appears in [9].

Another invariant of the hyperbolic structure that we compute is the maximal cusp volume. In the case of a 1-cusped hyperbolic 3-manifold, this is simply the volume of the largest possible cusp in the manifold. That is, every noncompact orientable finite volume hyperbolic 3-manifold decomposes along a disjoint set of tori to yield a compact piece and a finite set of cusps, each of which is topologically $T^{2} \times[0,1)$. Geometrically, each cusp is covered by an infinite set of disjoint horoballs in $H^{3}$, all of which are identified by the covering transformations. We expand the horoballs equivariantly until two first become tangent. The projection of these expanded horoballs down to $M$ is called the maximal cusp and its volume is the maximal cusp volume. Note that we still call it a cusp even though it touches itself on the boundary. In the case $M$ has more than one cusp, we define the maximal disjoint cusp volume to be the largest total volume of a set of cusps which have pairwise disjoint interiors, but which can touch each other or themselves on their boundaries.

The computer program currently can compute the maximal cusp volume of a single cusp in a multi-cusped manifold and the disjoint maximal cusp volume in a 2-cusped manifold. Table 1 lists the maximal cusp volume for each of the cusps in a given link.

A related invariant which is not listed in Table 1 is the cusp density of $M$. It is the ratio of the disjoint maximal cusp volume to the total volume. In [7], 
Meyerhoff utilized results of Boroczky [4] in order to prove that cusp density is at most $.853 \ldots$, a cusp density which is realized by the figure-eight knot complement. This upper bound on the cusp density can be used in conjunction with lower bounds on disjoint maximal cusp volume in order to obtain lower bounds for the volumes of manifolds as in [2] and [3].

Additional invariants of hyperbolic knot and link complements are obtainable from the cusps. Let $M$ be such a manifold, and let $C$ be a maximal cusp in $M$. Let $H$ be a horoball in $H^{3}$ which is centered at $\infty$ in the upper-half-space model of $H^{3}$ and which covers $C$. Normalize so that the boundary of $H$ is at height 1 above the $x-y$ plane. Then let $T$ be the parabolic isometry fixing $\infty$ which is the shortest translation. The column labelled "min trans" in Table 1 gives the length of that translation. This number can be thought of as the length of the shortest nontrivial parabolic loop in the boundary of the maximal cusp.

Continuing to let $\infty$ be the center of a horoball covering the cusp $C$, where we no longer assume $C$ is necessarily maximal, let $T_{1}$ and $T_{2}$ be the two covering transformations which fix $\infty$ and which are lifts of the meridian and longitude of the link component corresponding to $C$. Representing the $x-y$ plane as the complex plane, $T_{1}$ and $T_{2}$ are each translation by the complex numbers $a$ and $b$ respectively. Let $r$ be the ratio $a / b$. Then $r$ describes the parallelogram which is a fundamental domain for the action on the $x-y$ plane of the subgroup of isometries of $\pi_{1}(M)$ which fix $\infty$, the argument giving the angle between two of the sides and the magnitude giving the ratio of the lengths of two adjacent sides. We call this the homological cusp shape.

If instead, we choose $T_{1}$ to be the parabolic isometry which is the shortest translation among all the isometries of $\pi_{1}(M)$ which fix $\infty$, and $T_{2}$ to be the parabolic isometry which is the shortest translation linearly independent from $T_{1}$, then the ratio of the corresponding complex numbers is called the geometrical cusp shape. If $\operatorname{PGL}(2, \mathbf{Z})$ acts on either the homological or geometrical cusp shape as a fractional linear transformation, the single resulting equivalence class of complex numbers is called the conformal invariant of the cusp.

The next invariant that we examine is the horoball pattern itself. That is, for a one-cusped manifold, the maximal cusp is covered by horoballs, all of which have disjoint interiors and some of which are tangent to each other. Centering one of these horoballs at $\infty$, so that its boundary becomes a horizonal plane at Euclidean height 1, there will exist horoballs touching this plane, and hence having Euclidean diameter 1, as well as horoballs of smaller Euclidean diameter. The pattern of these horoballs is an invariant of the manifold. In practice the computer draws only the horoballs of Euclidean diameter greater than some constant $\alpha, 0<\alpha<1$. In Table $2, \alpha$ is 0.1 .

The computer locates the horoballs by the following procedure. A horoball is centered at each vertex of each of the tetrahedra in the finite set of tetrahedra that the manifold decomposes into. The computer determines the largest hyperbolic distance that any horoball reaches beyond the opposite face of a tetrahedron it is centered at the vertex of. Call this number $\beta$. By tiling the 
upper-half-space model of $H^{3}$ with lifts of the ideal tetradehdra from the previously computed triangulation of the manifold down to a level $\beta$ hyperbolic units below the Euclidean height $\alpha$, the computer will include all of the vertices corresponding to the centers of horoballs of Euclidean diameter greater than $\alpha$.

A similar pattern of horoballs is generated for multi-cusped manifolds by first maximizing one cusp and then maximizing the remaining cusps one at a time relative to the cusps which have already been expanded. Note that the result does not in general correspond to the maximal disjoint cusp volume, since there may be a choice of cusps containing the largest possible volume such that none of the cusps are themselves maximal.

Finally, the last invariant that the computer produces is the canonical triangulation. This triangulation can be thought of as dual to the Ford domain (cf. [6] or [10]). In the case we are dealing with a one-cusped manifold, we add in geodesic edges through the points of tangency of the maximal cusp and perpendicular to the cusp boundary. Then, we continue to expand the cusp through itself until a new point of tangency occurs. Again, we add in edges through points of tangency and perpendicular to the cusp boundary. Eventually, a canonical ideal cell division results. In most but not all cases, it is a triangulation. In unusually symmetric situations, some of the cells may not be tetrahedra. In principle, one could work with the cell division instead of a triangulation, but the current program is not set up to do so.

When a triangulation results, the computer checks it for symmetries. This yields the order of the isometry group of the manifold, given in the column labelled "num sym" in Table 1. In the case that the canonical cell division is not a triangulation, a question mark appears in this column.

The total hyperbolic volumes were calculated to an accuracy of $10^{-15}$. The other invariants were computed using the hyperbolic structures which gave the volumes to this accuracy.

\section{Applications}

The most useful application of these invariants is to tell hyperbolic 3-manifolds apart. Although volume tells the vast majority of hyperbolic manifolds apart, there are examples of arbitrarily large finite sets of distinct manifolds, all with the same volume (cf. [16]). Each of the other invariants mentioned can sometimes be used to distinguish between manifolds of the same volume. For example, the $5_{2}$ knot and a certain 12-crossing knot both have the same volume (cf. [15]), but their maximal cusp volumes are $1.973463 \ldots$ and $2.012733 \ldots$ respectively. More astonishing is the fact that the figure-eight knot complement has a "sibling" manifold which has the same volume, maximal cusp volume and horoball pattern. However the conformal invariants of the cusps differ.

There are several operations which we can perform on hyperbolic manifolds containing certain incompressible surfaces in order to obtain new hyperbolic manifolds with the same volume (cf. [1] and [12]). In certain of these situations, the resultant manifold will also have the same maximal cusp volume and 
cusp shape. Moreover, it can also have the same Chern-Simons invariant (cf. [8]). However, such manifolds can still often be distinguished by their horoball patterns. An example is the mutant knots displayed at the end of Table 2. Although none of the other invariants will distinguish between them, the horoball patterns tell them apart.

Cusp diagrams can also be used to obtain information about the isometries of a hyperbolic 3-manifold. For example, if a given cusp of a finite volume noncompact hyperbolic 3-manifold is preserved by an orientation-reversing isometry, there must be a fundamental domain for the action of the cusp subgroup which is either a rectangle or a rhombus. Thus Table 2 shows that relatively few of these knot complements double cover nonorientable manifolds.

Additionally, since an isometry of a 1-cusped hyperbolic 3-manifold must preserve the maximal cusp, such an isometry must lift to either a parabolic or elliptic isometry fixing $\infty$ in the upper-half-space model of $H^{3}$, where $\infty$ is chosen to be the center of a horoball covering the cusp. If the original isometry is fixed point free on the interior of the maximal cusp, then it must lift to a parabolic isometry which permutes the largest horoballs in the cusp diagram. Hence, for instance, since the cusp diagram for the knot $8_{10}$ admits no parabolic isometries other than those corresponding to its fundamental group, its complement has no fixed-point free isometries.

Define the periods of a knot as in Section 14D of [5]. Then the numbers of symmetries listed for 10-crossing knots in Table 1 of this paper imply that the only 10-crossing knots which could have odd periods are $10_{75}, 10_{123}$ and $10_{124}$. This contradicts the results in Table 1 of [5]. By examining the cusp diagrams themselves, one can show that some of the knots which appear with period 2 in that table in fact have no such period. 


\begin{tabular}{|c|c|c|c|c|c|c|}
\hline link & volume & $\begin{array}{l}\text { num } \\
\text { sym }\end{array}$ & $\begin{array}{c}\text { cusp } \\
\text { vol }\end{array}$ & $\begin{array}{c}\min \\
\text { trans }\end{array}$ & $\begin{array}{l}\text { homological } \\
\text { cusp shape }\end{array}$ & $\begin{array}{l}\text { geometrical } \\
\text { cusp shape }\end{array}$ \\
\hline 001 & $(2,3)$ torus knot & & & & & \\
\hline 4001 & 2.029883212819307 & 8 & 1.732051 & 1.000000 & $0.000000+0.288675 i$ & $0.000000+0.288675 i$ \\
\hline 5001 & $(2,5)$ torus knot & & & & & \\
\hline 3002 & 2.828122088330783 & 4 & 1.973464 & 1.150964 & $0.165153+0.197597 i$ & $0.053770+0.326785 i$ \\
\hline 6001 & 3.163963228883144 & 4 & 1.995452 & 1.247409 & $-0.184237+0.258674 i$ & $0.026219+0.388123 i$ \\
\hline 06002 & 4.400832516123046 & 4 & 2.882878 & 1.283754 & $0.116833+0.060607 i$ & $-0.020778+0.284311 i$ \\
\hline 6003 & 5.693021091281301 & 8 & 4.038067 & 1.210608 & $0.000000+0.181469 i$ & $0.000000+0.181469 i$ \\
\hline 07001 & $(2,7)$ torus knot & & & & & \\
\hline 107002 & 3.331744231641115 & 4 & 1.998818 & 1.300876 & $0.209974+0.238325 i$ & $0.014545+0.422820 i$ \\
\hline L107003 & 4.592125697027063 & 4 & 2.878339 & 1.340245 & $-0.102270+0.038195 i$ & $0.040097+0.306790 i$ \\
\hline 107004 & 5.137941201873418 & 8 & 3.538856 & 1.485116 & $-0.063371+0.298152 i$ & $0.030574+0.308593 i$ \\
\hline L107005 & 6.443537380850573 & 4 & $3.95545 ?$ & 1.222402 & $0.080637+0.045279 i$ & $0.015187+0.187658 i$ \\
\hline L107006 & 7.084925953510830 & 4 & 4.447335 & 1.226445 & $0.084410+0.089503 i$ & $-0.012041+0.168247 i$ \\
\hline L107007 & 7.643375172359955 & 8 & 5.065555 & 1.247409 & $0.038884+0.143017 i$ & $-0.005414+0.153398 i$ \\
\hline L108001 & 3.427205246274016 & 4 & 1.999601 & 1.332538 & $-0.219796+0.253214 i$ & $0.008867+0.443826 i$ \\
\hline 108002 & 4.935242678280654 & 4 & 2.761873 & 1.358235 & $0.084741+0.023099 i$ & $-0.001737+0.333968 i$ \\
\hline L108003 & 5.238684100798440 & 8 & 3.546273 & 1.526793 & $0.000000+0.328669 i$ & $0.000000+0.328669 i$ \\
\hline 108004 & 5.500486416347235 & 4 & 3.387435 & 1.509575 & $0.115347+0.045788 i$ & $0.053907+0.327490 i$ \\
\hline L108005 & 6.997189147792215 & 4 & 4.570602 & 1.605393 & $-0.081510+0.025954 i$ & $-0.011036+0.281509 i$ \\
\hline 108006 & 7.475237429505243 & 4 & 4.337089 & 1.249648 & $0.086770+0.113967 i$ & $0.007412+0.179725 i$ \\
\hline L108007 & 7.022196589095253 & 4 & 3.830256 & 1.252114 & $-0.101616+0.090266 i$ & $0.020703+0.202542 i$ \\
\hline L108008 & 7.801341224440063 & 4 & 4.551729 & 1.270490 & $0.063255+0.150773 i$ & $0.011462+0.176567 i$ \\
\hline L108009 & 7.588180223641627 & 8 & 5.181939 & 1.420609 & $0.000000+0.194727 i$ & $0.000000+0.194727 i$ \\
\hline L108010 & 8.651148558017082 & 2 & 4.948575 & 1.331926 & $-0.089564+0.086363 i$ & $0.006875+0.178982 i$ \\
\hline L108011 & 8.286316817806593 & 4 & 5.006547 & 1.276111 & $0.075585+0.111304 i$ & $0.004641+0.162500 i$ \\
\hline L108012 & 8.935856927486689 & 8 & 5.515651 & 1.232599 & $0.000000+0.137726 i$ & $0.000000+0.137726 i$ \\
\hline L108013 & 8.531232201460416 & 4 & 4.958060 & 1.266677 & $0.059708+0.135493 i$ & $-0.007225+0.161481 i$ \\
\hline 108014 & 9.217800316021929 & 4 & 5.634795 & 1.234788 & $0.057722+0.102920 i$ & $0.002660+0.135241 i$ \\
\hline L108015 & 9.930648293796183 & 4 & 6.219559 & 1.302105 & $0.067465+0.058505 i$ & $0.008518+0.135768 i$ \\
\hline L108016 & 10.579021916899270 & 2 & 7.177619 & 1.500953 & $0.078459+0.079682 i$ & $0.006741+0.156646 i$ \\
\hline L108017 & 10.985907606284820 & 2 & 7.250950 & 1.429835 & $0.000000+0.140977 i$ & $0.000000+0.140977 i$ \\
\hline L108018 & 12.350906209158200 & $?$ & 9.447478 & 1.553774 & $0.000000+0.127770 i$ & $0.000000+0.127770 i$ \\
\hline 08019 & $(3,4)$ torus knot & & & & & \\
\hline-108020 & 4.124903251807676 & 2 & 2.677040 & 1.576435 & $-0.188586+0.367343 i$ & $-0.022791+0.463038 i$ \\
\hline 8021 & 6.783713519835127 & 4 & 4.447881 & 1.372299 & $0.100739+0.138337 i$ & $0.019545+0.209877 i$ \\
\hline 109001 & $(2,9)$ torus knot & & & & & \\
\hline L109002 & 3.486660146295044 & 4 & 1.999840 & 1.352656 & $0.228083+0.245891 i$ & $0.005793+0.457383 i$ \\
\hline-109003 & 4.994856404125714 & 4 & 2.748613 & 1.374883 & $-0.073945+0.016714 i$ & $0.015779+0.343139 i$ \\
\hline 9004 & 5.556518816346559 & 4 & 3.376158 & 1.530728 & $0.112896+0.041754 i$ & $-0.024929+0.345211 i$ \\
\hline 9005 & 5.698441750940058 & 4 & 3.287842 & 1.522771 & $-0.055188+0.343778 i$ & $-0.055188+0.343778 i$ \\
\hline 9006 & 7.203600761623165 & 4 & 3.737575 & 1.258917 & $0.063830+0.021371$ & $0.003932+0.211946$ \\
\hline
\end{tabular}




\begin{tabular}{|c|c|c|c|c|c|c|}
\hline link & volume & $\begin{array}{l}\text { num } \\
\text { sym }\end{array}$ & $\begin{array}{c}\text { cusp } \\
\text { vol }\end{array}$ & $\underset{\text { trans }}{\min }$ & $\begin{array}{l}\text { homological } \\
\text { cusp shape }\end{array}$ & $\begin{array}{l}\text { geometrical } \\
\text { cusp shape }\end{array}$ \\
\hline 09007 & 8.014861457829954 & 4 & 4.474973 & 1.290478 & $0.087593+0.061681 i$ & $-0.012680+0.185204$ \\
\hline 109008 & 8.192347962434356 & 4 & 4.584343 & 1.308048 & $0.091040+0.072869 i$ & $-0.010586+0.186010 i$ \\
\hline 109009 & 8.016815565678016 & 4 & 4.894707 & 1.413912 & $0.060112+0.019570$ & $0.001723+0.204201 i$ \\
\hline 109010 & 8.773457282055681 & 8 & 5.556538 & 1.372324 & $-0.084412+0.077372 i$ & $-0.012505+0.168537 i$ \\
\hline 109011 & 8.288589042858491 & 4 & 165777 & 1.257194 & $5734+0.037744 i$ & $88492 i$ \\
\hline-109012 & 8.836642343885842 & 4 & 4.816076 & 1.280850 & $90 \mathrm{i}$ & $636 \mathrm{i}$ \\
\hline 109013 & 9.135094037937277 & 4 & 5.596681 & 1.38 & -0.083965 & 0.000379 \\
\hline-109014 & 8.954989262002549 & 4 & 4.768950 & 1.277111 & $0.069773+0.134921 i$ & 0.000706 \\
\hline L109015 & 9.885498660334439 & 4 & 5.673391 & 1.245774 & $-0.046418+0$ & $0.002594+0.136726 i$ \\
\hline L109016 & 9.883006960018827 & 4 & 5.901833 & 1.394529 & $-0.056519+0.022447 i$ & $0.164399 \mathrm{i}$ \\
\hline-109017 & 9224 & 8 & 18 & 53 & $065229 \mathrm{i}$ & 159886 \\
\hline-109018 & .057729635581000 & 4 & 4 & 239739 & 0.068594 & $-0 . c$ \\
\hline-109019 & .032547447836697 & 4 & 656 & 470 & $5467+0$ & $55 \mathrm{i}$ \\
\hline L109020 & 04073800876 & 4 & 039 & 1.3 & $6851+0.035594 i$ & 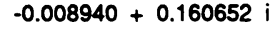 \\
\hline L109021 & 10.183265535681087 & 4 & 5.555841 & 1.2 & $-0.046101+c$ & $0.004873+0.138$ \\
\hline L109022 & 10.620727021239473 & 2 & 5.609470 & 1.318188 & $-0.077165+$ & -0.00063 \\
\hline L109023 & 52509 & 8 & 73 & 574 & $2954+0$ & -0 \\
\hline L109024 & 30 & & 4 & 9 & -0. & 0.0 \\
\hline L109025 & 1.390305147773655 & 2 & .303897 & 1.274099 & $0.046242+0.109168 i$ & 0.004798 \\
\hline L109026 & 0.595840514999372 & 4 & 5.911153 & 1.257366 & $-0.061935+0.092059 i$ & $-0.000553+0$ \\
\hline L109027 & 10.999980958287120 & 4 & 6.322088 & 1.253057 & $-0.019798+0.120939 i$ & -0.004900 \\
\hline L109028 & 16265123 & 4 & 471 & 1.312282 & $0.056909+0.081119 i$ & 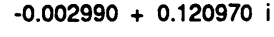 \\
\hline L109029 & 20 & 2 & 7 & 16 & 101 & $0.001328+0.151$ \\
\hline L10 & & & & & $\mathrm{i}$ & \\
\hline L109031 & 8105 & 8 & 806 & 231 & $051490+$ & 791 \\
\hline L109032 & 13.099899845892527 & 1 & 6.996783 & 1.312592 & $-0.055049+$ & $00261+0.123120 i$ \\
\hline L109033 & 13.280455636254788 & 1 & 7.245596 & 1.315237 & $-0.018742+0.116353 i$ & $-0.004970+0.119165 i$ \\
\hline L109034 & 14.344581387788963 & 2 & 8.681728 & 1.437423 & $-0.016411+0.116688 i$ & $-0.002574+0.118940 i$ \\
\hline L109035 & 311 & $?$ & 9511 & 1.664119 & $0.053241+0.272195 i$ & $-0.024405+0.280$ \\
\hline 6 & 5 & 2 & 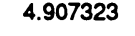 & 6 & 3004 & 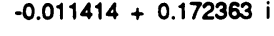 \\
\hline L109037 & 541 & 4 & 99 & 47 & -0.0 & -0 \\
\hline L109038 & 12.932858702252828 & 2 & 17565 & 7582 & $0.063316+0.073471 i$ & $-0.004407+0.127884 i$ \\
\hline L109039 & 12.810310003311115 & 2 & 7.598768 & 1.430371 & $-0.046696+0.115794 i$ & $0.000082+0.134625 i$ \\
\hline L109040 & 15.018342857865118 & $?$ & 10.810100 & 1.582347 & $0.051063+0.085209 i$ & 0.00234 \\
\hline 09041 & 12.0 & $?$ & $\mathbf{J}$ & 1.618034 & $-0.057417+$ & $156559 i$ \\
\hline L109042 & 4.056860224236820 & 2 & 2.363032 & 1.553898 & $-0.193265+0.088404 i$ & $-0.071362+0.500741 i$ \\
\hline L109043 & 5.904085858508103 & 2 & 3.152720 & 1.492733 & $-0.094798+0.027583 i$ & $0.033972+0.350089 i$ \\
\hline L109044 & 7.406767572367503 & 2 & 4.099116 & 1.363396 & $-0.037156+0.220476 i$ & $0.013154+0.225972 i$ \\
\hline L109045 & 8.602031166401501 & 2 & 5.000386 & 1.370048 & $0.070566+0.155709 i$ & $0.014517+0.186559 i$ \\
\hline L109046 & 4.751701965517900 & 4 & 3.274382 & 1.806879 & $-0.170121+0.431462 i$ & $0.051414+0.493179 i$ \\
\hline L109047 & 10.049957861274370 & ? & 6.687008 & 1.556030 & $-0.090501+0.092349 i$ & $-0.013466+0.180033$ \\
\hline 109048 & 9.531879835800978 & 12 & 6.153201 & 1.356203 & $-0.060034+0.119230 i$ & $-0.008216+0.14900$ \\
\hline
\end{tabular}

TABLE 1 (continued) 


\begin{tabular}{|c|c|c|c|c|c|c|}
\hline link & volume & $\begin{array}{l}\text { num } \\
\text { sym }\end{array}$ & $\begin{array}{c}\text { cusp } \\
\text { vol }\end{array}$ & $\min _{\text {trans }}$ & $\begin{array}{l}\text { homological } \\
\text { cusp shape }\end{array}$ & $\begin{array}{l}\text { geometrical } \\
\text { cusp shape }\end{array}$ \\
\hline 049 & 3627769277 & $?$ & 3814 & 703 & $62245 i$ & $0.010828+0.177648 i$ \\
\hline 10001 & 3.526195990735376 & 4 & 999927 & 366191 & $-0.232606+0.251540 i$ & $0.003988+0.466603 i$ \\
\hline 0002 & 5.114841460302401 & 4 & 09582 & 383682 & $0.064342+0.012135 i$ & $.001006+0.353295 i$ \\
\hline 0003 & 5.732104786782051 & 4 & 77220 & 34846 & $-0.037854+0.355381 i$ & $-0.037854+$ \\
\hline 0004 & .817129692825429 & 4 & 25693 & 1.530559 & $16717+0.042488 i$ & $55930+$ \\
\hline 005 & 373935134274568 & 4 & 678635 & 1.269457 & $-0.084501+0.039848 i$ & $0.015218+$ \\
\hline 10006 & 8.390937606724454 & 4 & 098807 & 1.262998 & $0.083567+0.047468 i$ & $0.001793+0.194572 i$ \\
\hline 10007 & 9.115906395629991 & 7 & 679422 & 1.284710 & $0.078054+0.129200 i$ & $0.013165+0.175367 i$ \\
\hline 10008 & 6.083234837064977 & 4 & 648369 & 1.658011 & $0.081024+0.018316 i$ & $-0.036275+0.373218 i$ \\
\hline 10009 & 8.294099675229289 & 4 & 4.749776 & 1.427084 & $-0.107153+0.110100 i$ & $0.020955+0.212318 i$ \\
\hline 10010 & 9.180573644138948 & 4 & 4.651176 & 1.282900 & $0.074937+0.135476 i$ & $0.003954+0.176838 i$ \\
\hline 10011 & 9.370442441399365 & 4 & 381980 & 1.380226 & $0.088210+0.081448 i$ & $0.003739+0.176903 i$ \\
\hline 10012 & 9.817495190695846 & 4 & 83588 & 1.316652 & -0.0731 & $4 i$ \\
\hline 10013 & 190464718 & 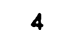 & 379859 & 1.244713 & -0.0343 & 0.004900 \\
\hline 10014 & 10.937689414417554 & 4 & 708017 & 1.258695 & $047361 i$ & $0.000214+$ \\
\hline 10015 & 8.973449326001361 & 1 & 272186 & 1.288936 & $-0.092010+0.065822 i$ & $-0.007145+0.194176 i$ \\
\hline 10016 & 9.546642305028446 & 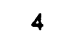 & 353217 & 1.385303 & $-0.089493+0.094427 i$ & $-0.009212+0.178769 i$ \\
\hline 10017 & 8.536755599193235 & 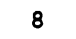 & 814171 & 1.594111 & $0.000000+0.218534 i$ & $0.000000+0.218534 i$ \\
\hline 10018 & 10.639844271257633 & 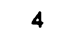 & 353108 & 1.245553 & $0.070988+0.086953 i$ & $-0.007665+0.144500 i$ \\
\hline 10019 & 9.844771302667735 & 4 & 973447 & 1.461467 & $.084374+0.059867 i$ & $-0.003731+0.178704 i$ \\
\hline 10020 & 718415007 & 4 & 536287 & 1.322309 & $0.087925+$ & $3295+0.191$ \\
\hline 10021 & 9.675 & 4 & 96 & 1.345687 & 0.0753 & -0.013674 \\
\hline 0022 & 9.981866517896332 & 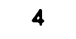 & 25 & 1.373750 & 0.0456 & -0.007670 \\
\hline 10023 & 11.393224634885061 & 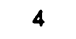 & 13160 & 1.256462 & -0.04858 & . 18072 \\
\hline 10024 & 4736821756 & 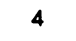 & 340201 & 1.267379 & 0.0302 & $-0.005883+0.137$ \\
\hline 10025 & 11.875779603652080 & 4 & 771023 & 1.291795 & 0.0598 & -0.003446 \\
\hline 10026 & 11.352017557987837 & 4 & 95615 & 1.393020 & $242556+$ & $-0.008319+0.136232 i$ \\
\hline 10027 & 966128570 & 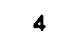 & 374558 & 1.266194 & $0.043509+$ & $-0.002146+0.116568 i$ \\
\hline 10028 & 06445 & 4 & 3038 & 8558 & $2 \mathrm{i}$ & $.003729+0.16$ \\
\hline 10029 & 246667982 & 4 & 383160 & 1.246061 & $063678+$ & $0.005872+0.129487 i$ \\
\hline 10030 & 369503651 & 4 & 23354 & 1.256795 & $8 \mathrm{i}$ & $-0.001158+0.128966 i$ \\
\hline 0031 & 3386 & 4 & 26 & & $2 \mathrm{i}$ & $0.138562 i$ \\
\hline 32 & & 4 & 27 & 572 & $-0.033924+C$ & $-0.006596+0.122862 i$ \\
\hline 0033 & 2333 & 8 & 3921 & 536 & $5 \mathrm{i}$ & $555 \mathrm{i}$ \\
\hline 10034 & 7868570 & 4 & 599796 & .334665 & 0.081 & -0.006049 \\
\hline 110035 & 10.394496785605322 & 4 & 899742 & 1.257273 & $0.031839+0.133821 i$ & $-0.006331+0.141112 i$ \\
\hline 110036 & 40305242 & 4 & 302790 & .262661 & $0.047931+0.123708 i$ & $-0.005595+0.142058$ \\
\hline 10037 & 10.96581 & 8 & 66148 & 261300 & $0.000000+0.131344 i$ & $0.000000+0.131344 i$ \\
\hline & & 4 & & & $0.031772+$ & -0.00005 \\
\hline & & 4 & & & & $0.126283 i$ \\
\hline & & 4 & & & $-0.037393+0.090814$ & $0.001390+0.106192 i$ \\
\hline & 661549863 & & & 05 & 0.073446 & 0.117519 \\
\hline
\end{tabular}

TABLE 1 (continued) 


\begin{tabular}{|c|c|c|c|c|c|c|}
\hline link & volume & $\begin{array}{l}\text { num } \\
\text { sym }\end{array}$ & $\begin{array}{c}\text { cusp } \\
\text { vol }\end{array}$ & $\begin{array}{c}\min \\
\text { trans }\end{array}$ & $\begin{array}{l}\text { homological } \\
\text { cusp shape }\end{array}$ & $\begin{array}{l}\text { geometrical } \\
\text { cusp shape }\end{array}$ \\
\hline 10042 & 13.239848366795648 & 4 & 7.457133 & 1.244272 & $0.002122+0.103764 i$ & $0.002122+0.103764 i$ \\
\hline 10043 & 12.602596114262077 & 8 & 7.306366 & 1.287780 & $0.000000+0.113489 i$ & $0.000000+0.113489 i$ \\
\hline 110044 & 12.968994205032500 & 4 & .300230 & 1.270313 & $0.053331+0.069743 i$ & $-0.000993+0.110515 i$ \\
\hline 110045 & 13.716075984752730 & 8 & 965675 & 1.261389 & $0.000000+0.099872 i$ & $0.000000+0.099872 i$ \\
\hline 10046 & 7.716999809324510 & 2 & 4.465802 & 1.649660 & $-0.062557+0.013436 i$ & $-0.025860+0.302480 i$ \\
\hline 10047 & 9.385194156727692 & 2 & 4.803283 & 1.372157 & $-0.078798+0.039737 i$ & $-0.004519+0.195888 i$ \\
\hline 110048 & 10.378901449017832 & 2 & 5.984340 & 1.453071 & $-0.007241+0.176114 i$ & $-0.007241+0.176114 i$ \\
\hline-110049 & 11.453193366219928 & 2 & 6.133251 & 1.321760 & $0.057143+0.028717 i$ & $-0.000580+0.142422 i$ \\
\hline L110050 & 11.198889792082566 & 2 & 6.098304 & 1.405292 & $-0.074955+0.050364 i$ & -0.0050 \\
\hline 10051 & 12.631379519944109 & 2 & 128129 & 1.332301 & $-0.044892+0.105386 i$ & $-0.006512+$ \\
\hline 10052 & 11.537548182498972 & 2 & 6.859008 & 1.481106 & $-0.078057+c$ & 0.005276 \\
\hline L110053 & 12.886847420183464 & 2 & 7.124076 & 1.327996 & 0.059399 & 0.000834 \\
\hline L110054 & 10.591311133639090 & 2 & 4.919714 & 1.315102 & $-0.086507+0.072377 i$ & $5555 \mathrm{i}$ \\
\hline L110055 & 12.185540627039736 & 2 & 390616 & 1.293867 & $0.064754+0.075282 i$ & -0.0074 \\
\hline L110056 & 12.398802155808716 & 2 & 172514 & 1.265472 & $-0.062955+c$ & 0.002 \\
\hline L110057 & 6049 & 2 & 6979 & .257089 & -0 . & 0. \\
\hline L110058 & 2.721330698537600 & 4 & 730189 & 1.297969 & $-0.022702+$ & 0.0 \\
\hline L110059 & 13.389944141432721 & 2 & 7.150058 & 1.285353 & $-0.054167+0.077840 i$ & -0.0 \\
\hline L110060 & 35708047 & 4 & 7.828635 & 1.299728 & $6599+0$ & -0. \\
\hline L110061 & 309 & 4 & 951932 & 1.695038 & $0414+0.024331 i$ & -0. \\
\hline L110062 & 512 & 2 & 68 & 411840 & $46 i$ & 0.0 \\
\hline L110063 & 952 & 4 & 0 & 70 & $6 i$ & 0. \\
\hline 64 & 94 & & 5 & & $i$ & -0 \\
\hline $\mathrm{L} 11 \mathrm{C}$ & 62 & 2 & & & $37 i$ & 08084 \\
\hline L110066 & 3.029268870834496 & 4 & 7.331201 & 1.325702 & $0.050934+0.028347 i$ & $-0.000141+0.119863 i$ \\
\hline L110067 & 12.421630505421152 & 2 & 6.319028 & 1.294082 & $0.041211+0.118131 i$ & $-0.006433+0.132195 i$ \\
\hline L110068 & 11.637035229302697 & 4 & 5.688419 & 1.342518 & $-0.066506+0.122240 i$ & $-0.010847+0.157677 i$ \\
\hline L110069 & 14.126505170670148 & 4 & 7.948534 & 1.286461 & $-0.033846+0.091600 i$ & $0.004875+0.1$ \\
\hline 70 & 12 & 2 & 2 & 2 & $i$ & -0. \\
\hline L110071 & 746039955 & 2 & 2 & 59 & $06630 i$ & $630 \mathrm{i}$ \\
\hline L110072 & 12.929594687049697 & 2 & 7.013903 & 1.278858 & $-0.057219+0.047151 i$ & $-0.005543+0.116324 i$ \\
\hline L110073 & 13.706880842371525 & 2 & 7.733839 & 1.269930 & $0.038306+0.087493 i$ & $0.002164+0.104219 i$ \\
\hline 10074 & 12.006036998378074 & 4 & 522540 & 13 & $0.035361+0.125296 i$ & $0.001578+c$ \\
\hline 10075 & 13.430748784284765 & 12 & 6875 & 1.326253 & $0.035010+$ & $0.002395+0.111037 i$ \\
\hline L110076 & 11.512860414469814 & 4 & 6.517227 & 1.323589 & $-0.063521+0.045266 i$ & $-0.007935+0.133934 i$ \\
\hline L110077 & 12.074711681444690 & 2 & 6.937139 & 1.311588 & $-0.048007+0.101220 i$ & $0.002686+0.123931 i$ \\
\hline L110078 & 12.502099196987689 & 4 & 7.194156 & 1.311687 & $0.057943+0.045046 i$ & $-0.003472+0.119477 i$ \\
\hline L110079 & 12.540295218484353 & 2 & 7.869607 & 1.491719 & $0.000000+0.141381 i$ & $0.000000+0.141381 i$ \\
\hline L110080 & 13.394044471515975 & 1 & 7.326246 & 1.362101 & $0.053897+0.030094 i$ & $0.002311+0.126579 i$ \\
\hline L110081 & 14.492667031583342 & 2 & 8.410048 & 1.347480 & $0.000000+0.107948 i$ & $0.000000+0.107948$ \\
\hline 110082 & 12.431479531326187 & 1 & 7.253723 & 1.465493 & $0.071436+0.093406 i$ & $0.003639+0.147950$ \\
\hline
\end{tabular}

TABLE 1 (continued) 


\begin{tabular}{|c|c|c|c|c|c|c|}
\hline link & volume & $\begin{array}{l}\text { num } \\
\text { sym }\end{array}$ & $\begin{array}{c}\text { cusp } \\
\text { vol }\end{array}$ & $\begin{array}{c}\min \\
\text { trans }\end{array}$ & $\begin{array}{l}\text { homological } \\
\text { cusp shape }\end{array}$ & $\begin{array}{l}\text { geometrical } \\
\text { cusp shape }\end{array}$ \\
\hline 083 & .258051849186285 & 1 & 27786 & 39222 & $6765+0$ & $0.001893+c$ \\
\hline 0084 & .709898781554164 & 1 & 442273 & 283946 & $-0.042292+0.091125 i$ & $-0.002336+0$ \\
\hline 10085 & 1.797773659928865 & 1 & 030088 & 542717 & $0.070858+0.038351 i$ & $-0.002427+0.169236 i$ \\
\hline 110086 & 4.341256139500095 & 1 & 801783 & 457273 & $0.031768+0.111594 i$ & $0.005226+0.120410 i$ \\
\hline 10087 & 14.273644598070369 & 1 & 007689 & 297011 & $0.026124+0.114044 i$ & $-0.001318+0.120$ \\
\hline 10088 & 5.646649171780129 & 2 & 43811 & 321543 & $0.000000+0.102207 i$ & $0+$ \\
\hline 10089 & 566058923120140 & & 2853 & 9 & $0+$ & .0 \\
\hline 10090 & 3.8661498632 & & 690 & 0 & r & 10 \\
\hline 0091 & 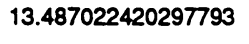 & 1 & .946395 & 0 & $-0.004578+0.137729 i$ & r \\
\hline 10092 & 6801 & 1 & 52590 & 7140 & i & $-0.003998+0.116255$ \\
\hline 10093 & 7686 & & 33014 & 492392 & $7095+c$ & $058+$ \\
\hline 110094 & 56652 & 1 & 75691 & 446023 & $8917+0$ & + \\
\hline 110095 & 352884581213 & & 668547 & 305733 & $9+$ & $t$ \\
\hline 096 & 2 & & 017805 & 2 & $3+c+c-1$ & + \\
\hline 097 & 95 & & 34367 & 583 & 101 & -0 . \\
\hline 098 & 258 & 2 & 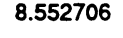 & 910 & 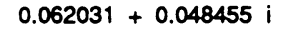 & $0.000194+0.127864 i$ \\
\hline 099 & 8 & & 6 & 64 & $000+c$ & + \\
\hline 10100 & 87 & & 9772 & 846 & $43+c$ & 1 \\
\hline 101 & 10 & & 3 & & $i$ & $4+0$ \\
\hline 02 & & & 2 & & $9+1$ & 1 \\
\hline 103 & 135 & & 8367 & 555 & $5 i$ & 81 \\
\hline 10104 & 4.107126687 & 2 & 910944 & .514165 & $479+0.128551 i$ & $9+$ \\
\hline 10105 & 695504 & 2 & 951634 & 354326 & $4875+c$ & + \\
\hline 106 & 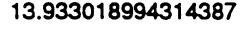 & & 2 & 280 & $9+c$ & +0.13 \\
\hline & & & & & i & c \\
\hline 08 & & & & & 91 & $34 i$ \\
\hline L110109 & 4.900208603026842 & & 380628 & .499964 & $0.000000+0$ & $0+c$ \\
\hline 10110 & 4.777462665 & & 88655 & 5189 & $0.060157+0$ & +0 \\
\hline & & & & & $\mathrm{i}$ & 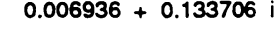 \\
\hline & & & & & 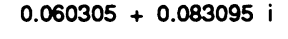 & 02 \\
\hline L110113 & 6.473473661468838 & & 110847 & 1.316549 & $-0.037011+0.087359 i$ & $-0.001185+$ \\
\hline L110114 & 15.304904343443041 & & 805784 & 1.543165 & $-0.026777+0.115203 i$ & $0.001265+0.12$ \\
\hline 10115 & 6.638038056436637 & & 955525 & 7190 & $0.000000+0.10$ & 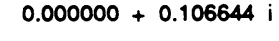 \\
\hline 16 & 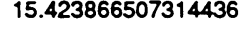 & & 3 & 20 & $0 \mathrm{i}$ & 4 \\
\hline 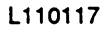 & 363 & . & 56 & 97 & $-0.041858+0$ & -0.0 \\
\hline 110118 & 17 & & 660452 & 68 & $0.000000+0.118038 i$ & $0.000000+$ \\
\hline L110119 & 15.938694126282648 & 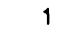 & 960135 & 1.438336 & $0.027716+0.108356 i$ & $0.002872+$ \\
\hline L110120 & 16.271368282159341 & & 469486 & 1.462641 & $0.051500+0.079667 i$ & $-0.003534+$ \\
\hline 110121 & 16.974877032190903 & 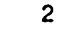 & 985171 & 1.461929 & $0.038244+0.090937 i$ & $-0.000806+0.107014$ \\
\hline 10122 & 8610278 & ( & .811343 & 1.578235 & $0.025070+0.109453 i$ & $-0.000154+0.115$ \\
\hline 23 & 17.085709482982861 & $?$ & 12.449491 & 1.618034 & 0.000000 & 0.000000 \\
\hline & 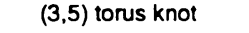 & & & & & \\
\hline
\end{tabular}

TABLE 1 (continued) 


\begin{tabular}{|c|c|c|c|c|c|c|}
\hline k & volume & $\begin{array}{l}\text { num } \\
\text { sym }\end{array}$ & $\begin{array}{c}\text { cusp } \\
\text { vol }\end{array}$ & $\underset{\text { trans }}{\min }$ & $\begin{array}{l}\text { homological } \\
\text { cusp shape }\end{array}$ & $\begin{array}{l}\text { geometrical } \\
\text { cusp shape }\end{array}$ \\
\hline 125 & 611961374497319 & 2 & 2315 & 316 & $.173433+$ & $-0.000 / 50+0.0102181$ \\
\hline 10126 & 6.904256123806481 & 2 & 072219 & 583497 & $.149777+0.118393$ & $.010330+0$. \\
\hline 127 & 8.896816759631431 & 2 & 903064 & 70967 & $090+0$. & $0.008574+0.191287 i$ \\
\hline 128 & .860539301740002 & 2 & 1092 & 339 & $74+0$ & $30+c$ \\
\hline 10129 & 8769 & 2 & 1494 & 76 & $22+c$ & $02+$ \\
\hline 0130 & .778198890046892 & 2 & 2387 & 9214 & $990+0.222583 i$ & . \\
\hline 131 & 5468 & 2 & 5099 & .466427 & $1008+0$ & -0.0 \\
\hline 10132 & .056860224236820 & 2 & 277288 & 553898 & $0.076367+$ & $0.076367+$ \\
\hline 10133 & .798300232166506 & 2 & 948709 & 367836 & $754+0.151493 i$ & $0.009499+0.23$ \\
\hline 10134 & .392922508336978 & 2 & 243108 & 7505 & $3800+0.022876 i$ & $8797+0$ \\
\hline 10135 & 68339628 & 2 & 05 & 45 & $619+0$ & $904+0.1$ \\
\hline 10136 & 546233128 & 4 & 2435 & 45 & $63+0$ & $70+c$ \\
\hline 37 & 874 & 2 & 65 & 1 & $91+0$ & +1 \\
\hline 138 & 898 & 4 & 1000 & 57 & $474+0$ & $+i$ \\
\hline 139 & 738 & 4 & 31 & 15 & $205+0$ & $253+0$. \\
\hline 40 & 545 & 2 & 782483 & & $25+$ & 0.4 \\
\hline 1 & 9 & 4 & 890544 & 6 & ( & \\
\hline 10142 & 6.770816780739823 & 4 & 800882 & 960 & $174+0.018267 i$ & $140+0$ \\
\hline 10143 & .070899270019318 & 2 & $2677^{\circ}$ & 587 & $116+0.1$ & $17+0$ \\
\hline 10144 & 3836 & 4 & 425854 & 589 & $0609+0$ & -0 \\
\hline 45 & 04 & 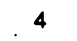 & 84 & 68 & $148+0$ & $70+0$. \\
\hline & 9 & & 323670 & & $597+0$. & $697+0.1$ \\
\hline L110 & & & & & $36+c$ & \\
\hline 10148 & 10.260243436313672 & 1 & 3885 & 77 & $0.078243+0.127096 i$ & $365+0.1$ \\
\hline 10149 & 969 & 1 & 16160 & 885 & $0.068245+0.050903 i$ & $387+0$ \\
\hline 0 & 818 & 1 & 21 & 18 & $304+0.0$ & 5 \\
\hline & & & & & $x^{-2}-5$ & \\
\hline 152 & 09 & 2 & & & $818+0.022474 i$ & -0 \\
\hline 10153 & 712 & 1 & 24 & 201 & $0.192559+0.272635 i$ & $800+0$ \\
\hline 110154 & 9.249887443861750 & 2 & 4363 & 3570 & $-0.097213+0.045980 i$ & $-0.025425+0$ \\
\hline 10155 & 2099 & & 88128 & 722084 & $0.078158+0.197629 i$ & $-0.014024+0.227674 i$ \\
\hline 6 & 26 & 2 & 81 & $3 / 4$ & $72+0.1$ & $3+0$ \\
\hline 7 & 12.66 & 8 & 394391 & 71 & $54+0.0$ & -0.0 \\
\hline 8 & 12.27 & & & & $0.024714+c$ & $0.006341+0$ \\
\hline 159 & 137913916 & & 262 & 73 & $0.059055+0.121130 i$ & $0.005655+0$ \\
\hline 10160 & 606090773 & & 82489 & 600169 & $-0.089574+0.041864 i$ & $-0.008853+0.233184 i$ \\
\hline 10161 & 48853054 & 2 & 38912 & 9792 & $0.110036+0.025661 i$ & $-0.090983+0.480266 i$ \\
\hline 10162 & 55 & 2 & 7 & 60 & $0.086573+0.096228 i$ & $1+0.17$ \\
\hline 3 & 13.25 & 2 & 4 & 1 & $0.095033 i$ & $670+0.132114$ \\
\hline 4 & 12.506687930892361 & 2 & 1 & 0 & $-0.011278+0.146426 i$ & $10300+0.146570$ \\
\hline .110100 & 11.603084649769077 & & 99 & 756 & 9227 & $0.006780+0.173997$ \\
\hline
\end{tabular}

TABLE 1 (continued) 


\begin{tabular}{|c|c|c|c|c|c|c|}
\hline link & volume & $\begin{array}{l}\text { num } \\
\text { sym }\end{array}$ & $\begin{array}{c}\text { cusp } \\
\text { vol }\end{array}$ & $\underset{\text { trans }}{\min }$ & $\begin{array}{l}\text { homological } \\
\text { cusp shape }\end{array}$ & $\begin{array}{l}\text { geometrical } \\
\text { cusp shape }\end{array}$ \\
\hline 00001 & $(2,0)$ torus link & & & & & \\
\hline L202001 & $(2,2)$ torus link & & & & & \\
\hline L204001 & $(2,4)$ torus link & & & & & \\
\hline \multirow[t]{2}{*}{ L205001 } & 3.663862376708876 & 8 & 2.000000 & 1.414214 & $0.250000+0.250000 i$ & $0.000000+0.500000 i$ \\
\hline & & & 2.000000 & 1.414214 & $0.250000+0.250000 i$ & $0.000000+0.500000 i$ \\
\hline L206001 & $(2,6)$ torus link & & & & & \\
\hline \multirow[t]{2}{*}{ L206002 } & 4.059766425638614 & 8 & 2.598076 & 1.732051 & $0.000000+0.577350 i$ & $0.000000+0.577350 i$ \\
\hline & & & 2.598076 & 1.732051 & $0.000000+0.577350 i$ & $0.000000+0.577350 i$ \\
\hline \multirow[t]{2}{*}{ L206003 } & 5.333489566898120 & 8 & 2.645751 & 1.414214 & $0.125000+0.330719 i$ & $0.000000+0.377964 i$ \\
\hline & & & 2.645751 & 1.414214 & $0.125000+0.330719 i$ & $0.000000+0.377964 i$ \\
\hline \multirow[t]{2}{*}{ L207001 } & 4.749499981874439 & 4 & 2.494874 & 1.774232 & $0.238631+0.109146 i$ & $0.170582+0.580771 i$ \\
\hline & & & 2.494874 & 1.774232 & $0.238631+0.109146 i$ & $0.170582+0.580771 i$ \\
\hline \multirow[t]{2}{*}{ L207002 } & 6.598951539438494 & 4 & 3.038735 & 1.524703 & $-0.079596+0.365165 i$ & $0.061281+0.372431 i$ \\
\hline & & & 3.038735 & 1.524703 & $-0.079596+0.365165 i$ & $0.061281+0.372431 i$ \\
\hline \multirow[t]{2}{*}{ L207003 } & 6.138138789085247 & 8 & 3.058171 & 1.550544 & $0.064266+0.382271 i$ & $0.064266+0.382271 i$ \\
\hline & & & 3.058171 & 1.550544 & $0.064266+0.382271 i$ & $0.064266+0.382271 i$ \\
\hline \multirow[t]{2}{*}{ L207004 } & 6.138138789085247 & 4 & 3.058171 & 1.550544 & $-0.118666+0.039868 i$ & $0.064266+0.382271 i$ \\
\hline & & & 3.058171 & 2.318603 & $-0.435734+0.382271 i$ & $-0.214709+0.822924 i$ \\
\hline \multirow[t]{2}{*}{ L207005 } & 7.706911802810127 & 4 & 3.538856 & 1.288009 & $0.096663+0.183464 i$ & $0.013570+0.233605 i$ \\
\hline & & & 2.935423 & 1.912843 & $-0.306675+0.366928 i$ & $-0.126743+0.596305 i$ \\
\hline \multirow[t]{2}{*}{ L207006 } & 8.997351943606996 & 4 & 4.645751 & 1.414214 & $0.098094+0.151906 i$ & $0.000000+0.215250 i$ \\
\hline & & & 4.645751 & 2.376079 & $0.125000+0.580719 i$ & $0.125000+0.580719 i$ \\
\hline \multirow{3}{*}{$\begin{array}{l}\text { L207007 } \\
\text { L207008 }\end{array}$} & same complement as & $s(2,4)$ & orus link & & & \\
\hline & 3.663862376708876 & 8 & 2.000000 & 1.414214 & $0.250000+0.250000 i$ & $0.000000+0.500000 i$ \\
\hline & & & 2.000000 & 1.414214 & $-0.750000+0.250000 i$ & $0.000000+0.500000 i$ \\
\hline L208001 & $(2,8)$ torus link & & & & & \\
\hline \multirow[t]{2}{*}{ L208002 } & 4.851170757332738 & 4 & 2.588550 & 1.852210 & $-0.330221+0.358443 i$ & $-0.160625+0.621127 i$ \\
\hline & & & 2.588550 & 1.852210 & $-0.330221+0.358443 i$ & $-0.160625+0.621127 i$ \\
\hline \multirow[t]{2}{*}{ L208003 } & 6.947555448592513 & 4 & 2.970785 & 1.550949 & $0.199155+0.166187 i$ & $-0.006544+0.404744 i$ \\
\hline & & & 2.970785 & 1.550949 & $0.199155+0.166187 i$ & $-0.006544+0.404744 i$ \\
\hline \multirow[t]{2}{*}{ L208004 } & 7.517689896474569 & 8 & 3.971262 & 1.744351 & $0.190172+0.214468 i$ & $0.045510+0.377612 i$ \\
\hline & & & 3.971262 & 1.744351 & $0.190172+0.214468 i$ & $0.045510+0.377612 i$ \\
\hline \multirow[t]{2}{*}{ L208005 } & 7.894594487233149 & 4 & 3.356610 & 1.553018 & $-0.128300+0.305365 i$ & $-0.021792+0.357944 i$ \\
\hline & & & 3.356610 & 1.553018 & $-0.128300+0.305365 i$ & $-0.021792+0.357944 i$ \\
\hline \multirow[t]{2}{*}{ L208006 } & 6.551743287887868 & 8 & 3.332295 & 1.672221 & $0.035597+0.416537 i$ & $0.035597+0.416537 i$ \\
\hline & & & 3.332295 & 1.672221 & $0.035597+0.416537 i$ & $0.035597+0.416537 i$ \\
\hline \multirow[t]{2}{*}{ L208007 } & 8.830664954907732 & 8 & 4.608220 & 1.682610 & $0.153258+0.143448 i$ & $0.044151+0.300705 i$ \\
\hline & & & 4.608220 & 1.682610 & $0.153258+0.143448 i$ & $0.044151+0.300705 i$ \\
\hline \multirow[t]{2}{*}{ L208008 } & $9.67280773 c$ & 8 & 4.760018 & 1.569379 & $0.000000+0.258712 i$ & $0.000000+0.2587 .12 i$ \\
\hline & & & 4.760018 & 1.569379 & $0.000000+0.258712 i$ & $0.000000+0.258712 i$ \\
\hline
\end{tabular}

TABLE 1 (continued) 


\begin{tabular}{|c|c|c|c|c|c|c|}
\hline link & volume & $\begin{array}{l}\text { num } \\
\text { sym }\end{array}$ & $\begin{array}{c}\text { cusp } \\
\text { vol }\end{array}$ & $\begin{array}{c}\min \\
\text { trans }\end{array}$ & $\begin{array}{c}\text { homological } \\
\text { cusp shape }\end{array}$ & $\begin{array}{l}\text { geometrical } \\
\text { cusp shape }\end{array}$ \\
\hline \multirow[t]{2}{*}{ L208009 } & 8.967360848788455 & 4 & 3.151133 & 226017 & $-0.393124+0.393892 i$ & $-0.159379+0.752492 i$ \\
\hline & & & 3.860417 & 1.277707 & $-0.091327+0.158983 i$ & $0.012618+0.210690 i$ \\
\hline \multirow[t]{2}{*}{ L208010 } & 9.659498544958967 & 4 & 4.397646 & 1.295233 & $-0.084911+0.138796 i$ & $-0.007531+0.190444 i$ \\
\hline & & & 3.110366 & 2.052618 & $-0.334911+0.388796 i$ & $-0.120611+0.655084 i$ \\
\hline \multirow[t]{2}{*}{ L208011 } & 8.793345603864991 & 4 & 3.104984 & 1.792059 & $-0.223781+0.388123 i$ & $-0.030623+0.515329 i$ \\
\hline & & & 4.412896 & 1.436625 & $-0.101957+0.059688 i$ & $-0.016573+0.232667 i$ \\
\hline \multirow[t]{2}{*}{ L208012 } & 9.659498544958967 & 4 & 3.110366 & 1.689575 & $-0.165089+0.388796 i$ & $0.015712+0.458357 i$ \\
\hline & & & 4.397646 & 1.295233 & $0.084911+0.138796 i$ & $0.007531+0.190444 i$ \\
\hline \multirow[t]{2}{*}{ L208013 } & 11.370774179519003 & 4 & 5.197823 & 1.288009 & $0.041386+0.148011 i$ & $-0.006302+0.159334 i$ \\
\hline & & & 4.935423 & 2.478103 & $0.056675+0.616928 i$ & $0.056675+0.616928 i$ \\
\hline \multirow[t]{2}{*}{ L208014 } & 10.666979133796239 & 8 & 5.291503 & 1.414214 & $0.000000+0.188982 i$ & $0.000000+0.188982 i$ \\
\hline & & & 5.291503 & 2.828427 & $0.250000+0.661438 i$ & $-0.250000+0.661438 i$ \\
\hline \multirow[t]{2}{*}{ L208015 } & 3.663862376708876 & 8 & 2.000000 & 1.414214 & $-0.250000+0.250000 i$ & $0.000000+0.500000 i$ \\
\hline & & & 2.000000 & 1.414214 & $-1.250000+0.250000 i$ & $0.000000+0.500000 i$ \\
\hline \multirow[t]{2}{*}{ L208016 } & 5.333489566898120 & 8 & 2.645751 & 1.414214 & $-0.875000+0.330719 i$ & $0.000000+0.377964 i$ \\
\hline & & & 2.645751 & 1.414214 & $-0.187500+0.165359 i$ & $0.000000+0.377964 i$ \\
\hline \multirow[t]{2}{*}{ L209001 } & 5.044899162918904 & 4 & 2.576252 & 1.887268 & $0.201372+0.064721 i$ & $-0.213097+0.617764 i$ \\
\hline & & & 2.576252 & 1.887268 & $0.201372+0.064721 i$ & $-0.213097+0.617764 i$ \\
\hline \multirow[t]{2}{*}{ L209002 } & 7.244320350873284 & 4 & 2.977959 & 1.594613 & $-0.206668+0.266916 i$ & $0.033766+0.424247 i$ \\
\hline & & & 2.977959 & 1.594613 & $-0.206668+0.266916 i$ & $0.033766+0.424247 i$ \\
\hline \multirow[t]{2}{*}{ L209003 } & 8.085586605502178 & 4 & 3.328190 & 1.580517 & $0.186729+0.206129 i$ & $0.056915+0.366444 i$ \\
\hline & & & 3.328190 & 1.580517 & $0.186729+0.206129 i$ & $0.056915+0.366444 i$ \\
\hline \multirow[t]{2}{*}{ L209004 } & 5.875918082667625 & 8 & 3.387794 & 2.209586 & $0.190996+0.054792 i$ & $-0.083185+0.710833 i$ \\
\hline & & & 3.387794 & 2.209586 & $0.190996+0.054792 i$ & $-0.083185+0.710833 i$ \\
\hline \multirow[t]{2}{*}{ L209005 } & 8.051684058915048 & 4 & 3.718663 & 1.751819 & $-0.163893+0.331636 i$ & $-0.033434+0.409904 i$ \\
\hline & & & 3.718663 & 1.751819 & $-0.163893+0.331636 i$ & $-0.033434+0.409904 i$ \\
\hline \multirow[t]{2}{*}{ L209006 } & 9.181187330616343 & 4 & 4.632118 & 1.733540 & $0.129336+0.260060 i$ & $-0.048022+0.317111 i i$ \\
\hline & & & 4.632118 & 1.733540 & $0.129336+0.260060 i$ & $-0.048022+0.317111 i$ \\
\hline \multirow[t]{2}{*}{ L209007 } & 10.394372662399081 & 4 & 4.639143 & 1.571823 & $0.101890+0.218842 i$ & $-0.017754+0.265092 i$ \\
\hline & & & 4.639143 & 1.571823 & $0.101890+0.218842 i$ & $-0.017754+0.265092 i$ \\
\hline \multirow[t]{2}{*}{ L209008 } & 8.584394654325494 & 4 & 3.640405 & 1.645018 & $-0.157688+0.284172 i$ & $-0.065890+0.359601 i$ \\
\hline & & & 3.640405 & 1.645018 & $-0.157688+0.284172 i$ & $-0.065890+0.359601 i$ \\
\hline \multirow[t]{2}{*}{ L209009 } & 9.417807631608254 & 8 & 3.896051 & 1.625815 & $0.169389+0.178320 i$ & $-0.022882+0.337674 i$ \\
\hline & & & 3.896051 & 1.625815 & $0.169389+0.178320 i$ & $-0.022882+0.337674 i$ \\
\hline \multirow[t]{2}{*}{ L209010 } & 6.784755786925872 & 8 & 3.510605 & 1.757383 & $0.021372+0.438826 i$ & $0.021372+0.438826 i$ \\
\hline & & & 3.510605 & 1.757383 & $0.021372+0.438826 i$ & $0.021372+0.438826 i$ \\
\hline \multirow[t]{2}{*}{ L209011 } & 10.759046640790302 & 4 & 4.752733 & 1.540936 & $0.116936+0.168791 i$ & $-0.014099+0.249004 i$ \\
\hline & & & 4.752733 & 1.540936 & $0.116936+0.168791 i$ & $-0.014099+0.249004 i$ \\
\hline \multirow[t]{2}{*}{ L209012 } & 11.188477802451692 & 4 & 5.002783 & 1.543639 & $0.041363+0.230735 i$ & $-0.013974+0.237327 i$ \\
\hline & & & 5.002783 & 1.543639 & $0.041363+0.230735 i$ & $-0.013974+0.237327 i$ \\
\hline \multirow[t]{2}{*}{ L209013 } & 872 & 4 & 3.510605 & 1.757383 & $-0.081143+0.015516 i$ & $0.021372+0.438826 i$ \\
\hline & & & 3.510605 & 2.597391 & $-0.478628+0.438826 i$ & $-0.122687+0.944937$ \\
\hline
\end{tabular}

TABLE 1 (continued) 


\begin{tabular}{|c|c|c|c|c|c|c|}
\hline link & volume & $\begin{array}{l}\text { num } \\
\text { sym }\end{array}$ & $\begin{array}{c}\text { cusp } \\
\text { vol }\end{array}$ & $\underset{\text { trans }}{\min }$ & $\begin{array}{l}\text { homological } \\
\text { cusp shape }\end{array}$ & $\begin{array}{l}\text { geometrical } \\
\text { cusp shape }\end{array}$ \\
\hline \multirow[t]{2}{*}{ L209014 } & 9.312341316558378 & 4 & 133314 & 597822 & $0.102011+0.053249 i$ & $-0.018219+0.247331 i$ \\
\hline & & & 219682 & .923424 & $0.263150+0.402460 i$ & $-0.045293+0.570929 i$ \\
\hline \multirow[t]{2}{*}{ L209015 } & 0.249194968003796 & 4 & 4.897249 & 1.394365 & $-0.098911+0.091027 i$ & $-0.018513+0.196763 i$ \\
\hline & & & 3.212249 & 2.193286 & $-0.373402+0.401531 i$ & $-0.131345+0.724979 i$ \\
\hline \multirow[t]{2}{*}{ L209016 } & 10.562806312097179 & 4 & 5.433666 & .1 .469902 & $-0.002242+0.198792 i$ & $42+$ \\
\hline & & & 3.209200 & 2.120137 & $-0.346432+0.401150 i$ & $-0.111373+0.682143 i$ \\
\hline \multirow[t]{2}{*}{ L209017 } & 9.665346419357368 & 4 & 3.345817 & 2.423900 & $-0.438511+0.418227 i$ & $-0.145466+0.853204 i$ \\
\hline & & & 3.957457 & 1.300343 & $0.030745+0.209114 i$ & $-0.014167+0.212690 i$ \\
\hline \multirow[t]{2}{*}{ L209018 } & 11.381786090554001 & 4 & 4.952088 & 1.254568 & $-0.079305+0.084395 i$ & $0.002196+0.158887 i$ \\
\hline & & & 3.163774 & 1.971666 & $-0.294226+0.395472 i$ & $-0.078316+0.604221 i$ \\
\hline \multirow[t]{2}{*}{ L209019 } & 7.453573250340333 & 2 & 3.081365 & 1.633868 & $-0.108812+0.029318 i$ & $0.078289+0$ \\
\hline & & & 3280 & 2.893673 & $-0.321104+0.129642 i$ & $0.253201+0.8$ \\
\hline \multirow[t]{2}{*}{ L209020 } & 09374947 & 2 & 321 & 326 & $0.054007+0.250395 i$ & $-0.012121+0.261481 i$ \\
\hline & & & 864094 & 2.292773 & $-0.276850+0.142556$ & $0.066417+0.673664 i$ \\
\hline \multirow[t]{2}{*}{ L209021 } & 9.814729698836974 & 2 & 4.345927 & 1.501793 & $-0.122543+0.087127 i$ & $56431 i$ \\
\hline & & & 368635 & 104637 & $0.162127+0.448337 i$ & $62+0$ \\
\hline \multirow[t]{2}{*}{ L209022 } & 10.956657816642180 & 2 & 4.346633 & 1.313444 & $0.099036+0.105303 i$ & $-0.010240+0.197915 i$ \\
\hline & & & 969596 & 1.880188 & $0.142193+0.393950 i$ & $-0.037286+0.442129 i$ \\
\hline \multirow[t]{2}{*}{ L209023 } & 80159587867 & 4 & 75 & 72 & $319+0.2$ & $19+c$ \\
\hline & & & 7275 & 1.912972 & $-0.180819+0.225792 i$ & $19+0.369282 i$ \\
\hline \multirow[t]{2}{*}{ L209024 } & 12.046092040094378 & $?$ & 6.363961 & 1.732051 & $0.111111+0.157135 i$ & $0.000000+0.235702 i$ \\
\hline & & & 6.363961 & 1.732051 & $0.111111+0.157135 i$ & $0.000000+0.235702 i$ \\
\hline \multirow[t]{2}{*}{ L209025 } & 11.381786090554001 & 4 & 4.952088 & 1.254568 & $-0.047482+0.143169 i$ & $96+0$ \\
\hline & & & 1774 & 1.783215 & $-c .205774+0.395472 i$ & $935+0$. \\
\hline \multirow[t]{2}{*}{ L209026 } & 17078181 & 4 & 1 & 15 & $224+C$ & $77+0$ \\
\hline & & & & & $3 i$ & $218+$ \\
\hline \multirow[t]{2}{*}{ L209027 } & 968003796 & 4 & 249 & 365 & $-0.096018+c$ & $0.018513+c$ \\
\hline & & & 3.212249 & .684063 & $-0.126598+0.401531 i$ & $0.054820+0.434530 i$ \\
\hline \multirow[t]{2}{*}{ L209028 } & 667712518 & 4 & 33458 & .321759 & $0.088194+0.119397 i$ & $0.000090+0.184542 i$ \\
\hline & & & 7 & 13 & $83 \mathrm{i}$ & $9+$ \\
\hline \multirow[t]{2}{*}{ L209029 } & 11.294969135798693 & 2 & 5.619131 & 1.473422 & $0.089107+0.059316 i$ & $-0.008324+0.192818 i$ \\
\hline & & & 3.751491 & 2.083512 & $-0.226744+0.468936 i$ & $0.054499+0$ \\
\hline \multirow[t]{2}{*}{ L209030 } & 12.276562777744445 & 2 & 5.768266 & 1.340367 & $-0.077857+0.078971 i$ & $-0.008000+0.155318 i$ \\
\hline & & & 979 & 2.114873 & $-0.289549+0.442385 i$ & $-0.014285+0.6$ \\
\hline \multirow[t]{2}{*}{ L209031 } & 10.215605664596744 & 4 & 3342 & 14214 & $0.097459+0.054794 i$ & $-0.010578+0.28$ \\
\hline & & & 95 & 686 & $0.214403+0.666537 i$ & $0.214403+0.666537 i$ \\
\hline \multirow[t]{2}{*}{ L209032 } & 380573867 & 4 & 6.276288 & .414214 & $0.017439+0.157398 i$ & $-0.007714+0.158955$ \\
\hline & & & 5.104984 & .554645 & $0.026219+0.638123 i$ & $0.026219+0.638123 i$ \\
\hline \multirow[t]{2}{*}{ L209033 } & 12.631223225497331 & 4 & 5.492952 & 1.277707 & $-0.027343+0.143389 i$ & $-0.006244+0.148340 i$ \\
\hline & & & 5.151133 & 2.638427 & $0.143124+0.643892 i$ & $0.143124+0.6$ \\
\hline \multirow[t]{2}{*}{ L209034 } & 11.9 & 1 & 65 & 2. & $0.221002+0.327263 i$ & $127+0.458390 i$ \\
\hline & & & 5.130584 & 1.449655 & $-0.024471+0.201834 i$ & $0.016994+0.203381$ \\
\hline
\end{tabular}

TABLE 1 (continued) 


\begin{tabular}{|c|c|c|c|c|c|c|}
\hline link & volume & $\begin{array}{l}\text { num } \\
\text { sym }\end{array}$ & $\begin{array}{c}\text { cusp } \\
\text { vol }\end{array}$ & $\underset{\text { trans }}{\min }$ & $\begin{array}{l}\text { homological } \\
\text { cusp shape }\end{array}$ & $\begin{array}{l}\text { geometrical } \\
\text { cusp shape }\end{array}$ \\
\hline \multirow[t]{2}{*}{ L209035 } & 11.373522432864332 & 1 & 42364 & 2.387652 & $0.261688+0.251973 i$ & $-0.004685+0.523709 i$ \\
\hline & & & 5.046845 & 1.547535 & $0.117772+0.104375 i$ & $-0.013706+0.236469 i$ \\
\hline \multirow[t]{2}{*}{ L209036 } & 11.471628355983366 & 4 & 5.703922 & 3.111040 & $0.310734+0.712990 i$ & $-0.299130+0.724994 i$ \\
\hline & & & 5.189791 & 1.414214 & $0.095575+0.108483 i$ & $-0.015772+0.191386 i$ \\
\hline \multirow[t]{2}{*}{ L209037 } & 11.471628355983366 & 4 & 5.703922 & 2.862289 & $0.060734+0.712990 i$ & $0.060734+0.712990 i$ \\
\hline & & & 5.189791 & 1.414214 & $0.076669+0.154685 i$ & $-0.015772+0.191386 i$ \\
\hline \multirow[t]{2}{*}{ L209038 } & 13.040401369708246 & 4 & 5.733463 & 1.288009 & $-0.055685+0.118509 i$ & $-0.005181+0.144488 i$ \\
\hline & & & 5.581174 & 2.803938 & $-0.068325+0.697647 i$ & $-0.068325+0.697647 i$ \\
\hline \multirow[t]{2}{*}{ L209039 } & 12.536170258246075 & 1 & & 2.079836 & $7971+c$ & $-0.017971+0.393495 i$ \\
\hline & & & 5.331931 & 1.497772 & $-0.105119+0.101501 i$ & $0.003405+0.210311 i$ \\
\hline \multirow[t]{2}{*}{ L209040 } & 12.046092040094378 & $?$ & 6.363961 & 1.732051 & $0.000000+0.235702 i$ & $0.000000+0.235702 i$ \\
\hline & & & 6.363961 & 2.449490 & $0.222222+0.157135 i$ & $0.000000+0.471405 i$ \\
\hline \multirow[t]{2}{*}{ L209041 } & 12.957429429593484 & 2 & 7.239870 & 2.168085 & $-0.065565+0.310801 i$ & $0.036433+0.320491 i$ \\
\hline & & & 009 & 1.564290 & $-0.092569+0.168935 i$ & $-0.023585+0.217097 i$ \\
\hline \multirow[t]{2}{*}{ L209042 } & 13.948417728284888 & 2 & 6.916088 & 1.624583 & $0.001141+0.190800 i$ & $0.001141+0.190800 i$ \\
\hline & & & 7.400834 & 2.278019 & $0.117866+0.305053 i$ & $0.012531+0.350145 i$ \\
\hline \multirow{3}{*}{$\begin{array}{l}\text { L209043 } \\
\text { L209044 }\end{array}$} & same complement as & is $(2,4) t$ & nk & & & \\
\hline & 6.138138789085247 & 4 & 88171 & 1.550544 & $0.118666+c$ & $-0.064266+0.382271 i$ \\
\hline & & & 71 & 2.318603 & $-0.564266+0.382271 i$ & $0.214709+0.822924 i$ \\
\hline \multirow[t]{2}{*}{ L209045 } & 5.333489566898120 & 8 & 2.645751 & 1.414214 & $-0.156250+0.082680 i$ & $0.000000+0.377964 i$ \\
\hline & & & 2.645751 & 1.414214 & $-1.125000+0.330719 i$ & $0.000000+0.377964 i$ \\
\hline \multirow[t]{2}{*}{ L209046 } & 6.138138789085247 & 8 & 3.058171 & 1.550544 & $0.064266+0.382271 i$ & $0.064266+0.382271 i$ \\
\hline & & & 171 & 1.550544 & $-0.935734+0.382271 i$ & $0.064266+0.382271 i$ \\
\hline \multirow[t]{2}{*}{$\llcorner 209047$} & 3.663862376708876 & 8 & 00 & 1.4 & $0.250000+0.250000 i$ & $0.000000+0.500000 i$ \\
\hline & & & 000 & 1214 & $-1.750000+0.250000 i$ & $0.000000+0.500000 i$ \\
\hline \multirow[t]{2}{*}{ L209048 } & 7.706911802810127 & 4 & 3.538856 & 1.288009 & $-0.109157+0.074538 i$ & $-0.013570+0.233605 i$ \\
\hline & & & 2.935423 & 1.912843 & $-0.693325+0.366928 i$ & $0.126743+0.596305 i$ \\
\hline & same complement as & as $(2,6)$ & torus link & & & \\
\hline \multirow[t]{2}{*}{ L209050 } & 4.415332477453866 & 4 & 110 & 1.68 & $0.296097+0.388986 i$ & $0.088302+0.601410 i$ \\
\hline & & & 0 & 1.6 & $-0.423371+0.071724 i$ & $0.088302+0.601410 i$ \\
\hline \multirow[t]{2}{*}{ L209051 } & 5.978240565858970 & 2 & 2.895313 & 1.742197 & $-0.172112+0.064435 i$ & $-0.026289+0.522844 i$ \\
\hline & & & 2.929333 & 2.226017 & $0.365309+0.635931 i$ & $-0.213752+0.787783 i$ \\
\hline \multirow[t]{2}{*}{ L209052 } & 8.209021220809309 & 2 & 3.461056 & 1.364670 & $0.134022+0.122959 i$ & $0.003716+0.268988 i$ \\
\hline & & & 3.349059 & 2.052447 & $0.200330+0.556843 i$ & $-0.157840+0.586431 i$ \\
\hline \multirow{3}{*}{ L209054 } & saleminte or $(2,4)$ lorus & & & & & \\
\hline & 7.517689896474569 & 4 & 56 & 1.958565 & $-0.082012+0.447278 i$ & $-0.082012+0.447278 i$ \\
\hline & & & 4.148656 & 1.958565 & $-0.082012+0.447278 i$ & $-0.082012+0.447278 i$ \\
\hline \multirow[t]{2}{*}{ L209055 } & 8.997351943606996 & 4 & 4.645751 & 1.414214 & $0.099174+0.065820 i$ & $0.000000+0.215250 i$ \\
\hline & & & 4.645751 & 2.765095 & $-0.375000+0.580719 i$ & $0.141318+0.797845 i$ \\
\hline \multirow[t]{2}{*}{ L209056 } & 606996 & 4 & 4.645751 & 1.414214 & $-0.099174+0.065820 i$ & $0.000000+0.215250 i$ \\
\hline & & & 4.645751 & 2.765095 & $-0.625000+0.580719 i$ & $-0.141318+0.797845 i$ \\
\hline
\end{tabular}

TABLE 1 (continued) 


\begin{tabular}{|c|c|c|c|c|c|c|}
\hline link & volume & $\begin{array}{l}\text { num } \\
\text { sym }\end{array}$ & $\begin{array}{c}\text { cusp } \\
\text { vol }\end{array}$ & $\begin{array}{c}\min \\
\text { trans }\end{array}$ & $\begin{array}{l}\text { homological } \\
\text { cusp shape }\end{array}$ & $\begin{array}{l}\text { geometrical } \\
\text { cusp shape }\end{array}$ \\
\hline \multirow[t]{2}{*}{ L209057 } & 8.355502146379566 & 2 & 4.269296 & 1.682230 & $0.165226+0.153022 i$ & $0.028124+0.329020 i$ \\
\hline & & & 4.227473 & 2.289107 & $0.048321+0.615966 i$ & $0.048321+0.615966 i$ \\
\hline \multirow[t]{2}{*}{ L209058 } & 9.966511883698373 & 2 & 4.634835 & 1.405887 & $-0.072474+0.184802 i$ & $0.007300+0.212974 i$ \\
\hline & & & 3.988781 & 2.041189 & $-0.108645+0.498598 i$ & $-0.108645+0.498598 i$ \\
\hline \multirow[t]{2}{*}{ L209059 } & 5.333489566898120 & 2 & 2.645751 & 2.000000 & $-1.000000+1.322876 i$ & $0.000000+0.755929 i$ \\
\hline & & & 3.307189 & 2.000000 & $0.133178+0.030908 i$ & $0.045455+0.601307 i$ \\
\hline \multirow[t]{2}{*}{ L209060 } & 5.333489566898120 & 2 & 2.645751 & 2.000000 & $2.000000+1.322876 i$ & $0.000000+0.755929 i$ \\
\hline & & & 3.307189 & 2.000000 & $-0.250000+0.132288 i$ & $-0.045455+0.601307 i$ \\
\hline & satellite of $(2,4)$ torus & $s$ link & & & & \\
\hline \multirow{3}{*}{ L306001 } & 5.333489566898120 & $?$ & 2.645751 & 2.000000 & $-0.375000+0.330719 i$ & $0.250000+0.661438 i$ \\
\hline & & & 2.645751 & 2.000000 & $-0.375000+0.330719 i$ & $0.250000+0.661438 i$ \\
\hline & & & 2.645751 & 2.000000 & $-0.375000+0.330719 i$ & $0.250000+0.661438 i$ \\
\hline \multirow[t]{3}{*}{ L306002 } & 7.327724753417752 & $?$ & 4.000000 & 2.000000 & $0.000000+0.500000 i$ & $0.000000+0.500000 i$ \\
\hline & & & 4.000000 & 2.000000 & $0.000000+0.500000 i$ & $0.000000+0.500000 i$ \\
\hline & & & 4.000000 & 2.000000 & $0.000000+0.500000 i$ & $0.000000+0.500000 i$ \\
\hline L306003 & $(3,3)$ torus link & & & & & \\
\hline \multirow[t]{3}{*}{ L307001 } & 7.706911802810127 & 12 & 2.935423 & 1.658967 & $-0.193325+0.366928 i$ & $-0.027140+0.467210 i$ \\
\hline & & & 2.935423 & 1.658967 & $-0.193325+0.366928 i$ & $-0.027140+0.467210 i$ \\
\hline & & & 2.935423 & 1.658967 & $-0.193325+0.366928 i$ & $-0.027140+0.467210 i$ \\
\hline \multirow[t]{3}{*}{ L308001 } & 6.551743287887868 & 4 & 3.332295 & 2.495350 & $-0.464403+0.416537 i$ & $-0.163413+0.904793 i$ \\
\hline & & & 3.710131 & 2.495350 & $-0.295146+0.121359 i$ & $0.071194+0.833074 i$ \\
\hline & & & 3.710131 & 2.495350 & $-0.295146+0.121359 i$ & $0.071194+0.833074 i$ \\
\hline \multirow[t]{3}{*}{ L308002 } & 8.793345603864991 & 4 & 3.104984 & 1.905514 & $-0.276219+0.388123 i$ & $-0.073066+0.575425 i$ \\
\hline & & & 3.881782 & 1.905514 & $0.130534+0.427872 i$ & $-0.074096+0.455646 i$ \\
\hline & & & 3.881782 & 1.905514 & $0.130534+0.427872 i$ & $-0.074096+0.455646 i$ \\
\hline \multirow[t]{3}{*}{ L308003 } & 8.967360848788455 & 12 & 3.151133 & 1.632535 & $-0.106876+0.393892 i$ & $0.062653+0.413395 i$ \\
\hline & & & 3.151133 & 1.632535 & $-0.106876+0.393892 i$ & $0.062653+0.413395 i$ \\
\hline & & & 3.151133 & 1.632535 & $-0.106876+0.393892 i$ & $0.062653+0.413395 i$ \\
\hline \multirow[t]{3}{*}{ L308004 } & 10.149416064096536 & 8 & 6.928203 & 2.000000 & $0.000000+0.288675 i$ & $0.000000+0.288675 i$ \\
\hline & & & 3.464102 & 2.000000 & $-0.250000+0.433013 i$ & $0.000000+0.577350 i$ \\
\hline & & & 3.464102 & 2.000000 & $0.250000+0.433013 i$ & $0.000000+0.577350 i$ \\
\hline \multirow[t]{3}{*}{ L308005 } & 9.802001165794123 & 4 & 5.058171 & 2.635949 & $0.185734+0.632271 i$ & $0.185734+0.632271 i$ \\
\hline & & & 4.544039 & 2.000000 & $0.213849+0.272020 i$ & $-0.041063+0.436272 i$ \\
\hline & & & 4.544039 & 2.000000 & $0.213849+0.272020 i$ & $-0.041063+0.436272 i$ \\
\hline \multirow[t]{3}{*}{ L308006 } & 10.666979133796239 & $?$ & 5.291503 & 2.645751 & $0.000000+0.661438 i$ & $0.000000+0.661438 i$ \\
\hline & & & 5.291503 & 2.000000 & $0.000000+0.377964 i$ & $0.000000+0.377964 i$ \\
\hline & & & 5.291503 & 2.000000 & $0.000000+0.377964 i$ & $0.000000+0.377964 i$ \\
\hline \multirow{2}{*}{$\begin{array}{l}\text { L308007 } \\
\text { L308008 }\end{array}$} & $\infty n$ & Du & ure & cre & & \\
\hline & 5.333489566898120 & $?$ & 2.645751 & 2.000000 & $0.250000+0.661438 i$ & $-0.250000+0.661438 i$ \\
\hline \multirow{2}{*}{$L 308008$} & & & 2.645751 & 2.000000 & $0.250000+0.661438 i$ & $-0.250000+0.661438 i$ \\
\hline & & & 2.645751 & 2.000000 & $-0.625000+0.330719 i$ & $0.250000+0.661438$ \\
\hline
\end{tabular}

TABLE 1 (continued) 


\begin{tabular}{|c|c|c|c|c|c|c|}
\hline link & volume & $\begin{array}{l}\text { num } \\
\text { sym }\end{array}$ & $\begin{array}{c}\text { cusp } \\
\text { vol }\end{array}$ & $\underset{\text { trans }}{\min }$ & $\begin{array}{l}\text { homological } \\
\text { cusp shape }\end{array}$ & $\begin{array}{l}\text { geometrical } \\
\text { cusp shape }\end{array}$ \\
\hline \multirow[t]{3}{*}{ L308009 } & 7.327724753417752 & $?$ & 4.000000 & 2.000000 & $0.000000+0.500000 i$ & $0.000000+0.500000 i$ \\
\hline & & & 4.000000 & 2.828427 & $-0.500000+0.500000 i$ & $0.000000+1.000000 i$ \\
\hline & & & 4.000000 & 2.000000 & $0.000000+0.500000 i$ & $0.000000+0.500000 i$ \\
\hline L308010 & satellite of $(2,4)$ torus & s link & & & & \\
\hline \multirow[t]{3}{*}{ L309001 } & 10.740257667712518 & 4 & 4.134788 & 1.747048 & $-0.183581+0.165725 i$ & $-0.000181+0.369085 i$ \\
\hline & & & 3.274267 & 2.303964 & $-0.405281+0.409283 i$ & $-0.141049+0.785266 i$ \\
\hline & & & 4.134788 & 1.747048 & $-0.183581+0.165725 i$ & $-0.000181+0.369085 i$ \\
\hline \multirow[t]{3}{*}{ L309002 } & 11.762234287078181 & 4 & 4.234103 & 1.607862 & $0.017754+0.304249 i$ & $0.017754+0.304249 i$ \\
\hline & & & 3.169903 & 2.036866 & $-0.319839+0.396238 i$ & $-0.097701+0.639482 i$ \\
\hline & & & 4.234103 & 1.607862 & $0.017754+0.304249 i$ & $0.017754+0.304249 i$ \\
\hline \multirow[t]{3}{*}{ L309003 } & 11.294969135798693 & 4 & 3.751491 & 2.170974 & $-0.273256+0.468936 i$ & $0.028492+0.626872 i$ \\
\hline & & & 3.751491 & 2.170974 & $-0.273256+0.468936 i$ & $0.028492+0.626872 i$ \\
\hline & & & 4.045339 & 1.502339 & $-0.114612+0.059988 i$ & $0.011741+0.278471 i$ \\
\hline \multirow[t]{3}{*}{ L309004 } & $12.276562 \pi 77744445$ & 4 & 3.539079 & 1.959568 & $-0.210451+0.442385 i$ & $0.036068+0.540092 i$ \\
\hline & & & 3.539079 & 1.959568 & $-0.210451+0.442385 i$ & $0.036068+0.540092 i$ \\
\hline & & & 4.112431 & 1.340367 & $0.102653+0.146508 i$ & $0.009888+0.217985 i$ \\
\hline \multirow[t]{3}{*}{ L309005 } & 9.312341316558378 & 4 & 3.219682 & 1.867927 & $-0.236850+0.402460 i$ & $-0.025226+0.540670 i$ \\
\hline & & & 3.507769 & 1.867927 & $-0.234221+0.165133 i$ & $0.036439+0.494662 i$ \\
\hline & & & 3.507769 & 1.867927 & $-0.234221+0.165133 i$ & $0.036439+0.494662 i$ \\
\hline \multirow[t]{3}{*}{ L309006 } & 10.562806312097179 & 4 & 3.712042 & 1.718159 & $0.139305+0.340671 i$ & $0.004485+0.397584 i$ \\
\hline & & & 3.712042 & 1.718159 & $0.139305+0.340671 i$ & $0.004485+0.397584 i$ \\
\hline & & & 3.209200 & 1.718159 & $-0.153568+0.401150 i$ & $0.035260+0.457220 i$ \\
\hline \multirow[t]{3}{*}{ L309007 } & 9.665346419357368 & $?$ & 3.345817 & 1.690893 & $-0.061489+0.418227 i$ & $-0.061489+0.418227 i$ \\
\hline & & & 3.345817 & 1.690893 & $-0.061489+0.418227 i$ & $-0.061489+0.418227 i$ \\
\hline & & & 3.345817 & 1.690893 & $-0.061489+0.418227 i$ & $-0.061489+0.418227 i$ \\
\hline \multirow[t]{3}{*}{ L.309008 } & 11.751836165335249 & 4 & 3.387794 & 1.790834 & $0.145301+0.423474 i$ & $-0.060606+0.465438 i$ \\
\hline & & & 6.775589 & 1.790834 & $-0.030303+0.232719 i$ & $0.024911+0.234013 i$ \\
\hline & & & 3.387794 & 2.209586 & $-0.354699+0.423474 i$ & $-0.083185+0.710833 i$ \\
\hline \multirow[t]{3}{*}{ L309009 } & 11.751836165335249 & 8 & 6.775589 & 1.790834 & $-0.118009+0.109585 i$ & $0.024911+0.234013 i$ \\
\hline & & & 3.387794 & 2.209586 & $-0.354699+0.423474 i$ & $-0.083185+0.710833 i$ \\
\hline & & & 3.387794 & 2.209586 & $-0.354699+0.423474 i$ & $-0.083185+0.710833 i$ \\
\hline \multirow[t]{3}{*}{ L309010 } & 13.323360921667843 & 4 & 5.096031 & 1.677630 & $0.102236+0.230867 i$ & $-0.029865+0.272872 i$ \\
\hline & & & 5.110366 & 2.577657 & $0.084911+0.638796 i$ & $0.084911+0.638796 i$ \\
\hline & & & 5.096031 & 1.677630 & $0.102236+0.230867 i$ & $-0.029865+0.272872 i$ \\
\hline \multirow[t]{3}{*}{ L309011 } & 13.040401369708246 & 4 & 4.755814 & 1.658967 & $0.031118+0.285962 i$ & $0.031118+0.285962 i$ \\
\hline & & & 5.581174 & 2.883655 & $0.181675+0.697647 i$ & $0.181675+0.697647 i$ \\
\hline & & & 4.755814 & 1.658967 & $0.031118+0.285962 i$ & $0.031118+0.285962 i$ \\
\hline \multirow[t]{3}{*}{ L309012 } & 13.813278440805412 & 4 & 5.464102 & 1.414214 & $0.000000+0.183013 i$ & $0.000000+0.183013 i$ \\
\hline & & & 5.464102 & 2.732051 & $0.000000+0.683013 i$ & $0.000000+0.683013 i$ \\
\hline & & & 3.464102 & 2.000000 & $-0.250000+0.433013 i$ & $0.000000+0.577350$ \\
\hline
\end{tabular}

TABLE 1 (continued) 


\begin{tabular}{|c|c|c|c|c|c|c|}
\hline link & volume & $\begin{array}{l}\text { num } \\
\text { sym }\end{array}$ & $\begin{array}{c}\text { cusp } \\
\text { vol }\end{array}$ & $\underset{\text { trans }}{\min }$ & $\begin{array}{l}\text { homological } \\
\text { cusp shape }\end{array}$ & $\begin{array}{l}\text { geometrical } \\
\text { cusp shape }\end{array}$ \\
\hline \multirow[t]{3}{*}{ L309013 } & 5.333489566898120 & $?$ & 2.645751 & 2.000000 & $-0.312500+0.165359 i$ & $0.250000+0.661438 i$ \\
\hline & & & 2.645751 & 2.000000 & $-1.375000+0.330719 i$ & $0.250000+0.661438 i$ \\
\hline & & & 2.645751 & 2.000000 & $-0.312500+0.165359 i$ & $-0.250000+0.661438 i$ \\
\hline \multirow[t]{3}{*}{ L309014 } & 7.706911802810127 & 12 & 2.935423 & 1.658967 & $0.027140+0.467210 i$ & $0.027140+0.467210 i$ \\
\hline & & & 2.935423 & 1.658967 & $-0.806675+0.366928 i$ & $0.027140+0.467210 i$ \\
\hline & & & 2.935423 & 1.658967 & $0.027140+0.467210 i$ & $0.027140+0.467210 i$ \\
\hline \multirow[t]{3}{*}{ L309015 } & 8.355502146379566 & 4 & 4.227473 & 2.829898 & $-0.548321+0.615966 i$ & $-0.193729+0.905739 i$ \\
\hline & & & 2.987721 & 1.682230 & $-0.140003+0.045821 i$ & $-0.096864+0.452870 i$ \\
\hline & & & 4.227473 & 2.829898 & $-0.548321+0.615966 i$ & $-0.193729+0.905739 i$ \\
\hline \multirow[t]{3}{*}{ L309016 } & 9.966511883698373 & 4 & 3.988781 & 2.535377 & $-0.391355+0.498598 i$ & $0.016805+0.805427 i$ \\
\hline & & & 3.408369 & 1.405887 & $0.142020+0.174101 i$ & $-0.015646+0.289104 i$ \\
\hline & & & 3.988781 & 2.535377 & $-0.391355+0.498598 i$ & $0.016805+0.805427 i$ \\
\hline \multirow[t]{3}{*}{ L309017 } & 5.333489566898120 & $?$ & 2.645751 & 2.000000 & $1.500000+1.322876 i$ & $-0.250000+0.661438 i$ \\
\hline & & & 2.645751 & 2.000000 & $-0.250000+0.094491 i$ & $0.250000+0.661438 i$ \\
\hline & & & 2.645751 & 2.000000 & $1.500000+1.322876 i$ & $0.250000+0.661438 i$ \\
\hline \multirow[t]{3}{*}{ L309018 } & 7.327724753417752 & $?$ & 4.000000 & 2.000000 & $0.000000+0.500000 i$ & $0.000000+0.500000 i$ \\
\hline & & & 4.000000 & 2.000000 & $0.000000+0.500000 i$ & $0.000000+0.500000 i$ \\
\hline & & & 4.000000 & 2.000000 & $-1.000000+0.500000 i$ & $0.000000+0.500000 i$ \\
\hline \multirow[t]{3}{*}{ L309019 } & 7.327724753417752 & $?$ & 4.000000 & 2.828427 & $0.500000+0.500000 i$ & $0.000000+1.000000 i$ \\
\hline & & & 4.000000 & 2.000000 & $1.000000+0.500000 i$ & $0.000000+0.500000 i$ \\
\hline & & & 4.000000 & 2.000000 & $0.200000+0.100000 i$ & $0.000000+0.500000 i$ \\
\hline \multirow[t]{3}{*}{ L309020 } & 10.991587130126628 & 4 & 4.000000 & 1.414214 & $0.000000+0.250000 i$ & $0.000000+0.250000 i$ \\
\hline & & & 6.000000 & 3.162278 & $-0.250000+0.750000 i$ & $-0.250000+0.750000 i$ \\
\hline & & & 20 & 828427 & $-0.500000+0.500000 i$ & $0.000000+1.000000$ \\
\hline \multirow{5}{*}{ L408001 } & satellite of Whitehea & ad link & & & & \\
\hline & 10.149416064096536 & $?$ & 3.464102 & 2.000000 & $-0.250000+0.433013 i$ & $0.000000+0.577350 i$ \\
\hline & & & 3.464102 & 2.000000 & $-0.250000+0.433013 i$ & $0.000000+0.577350 i$ \\
\hline & & & 3.464102 & 2.000000 & $-0.250000+0.433013 i$ & $0.000000+0.577350 i$ \\
\hline & & & 3.464102 & 2.000000 & $-0.250000+0.433013 i$ & $0.000000+0.577350 i$ \\
\hline \multirow[t]{4}{*}{ L408002 } & 7.327724753417752 & 64 & 4.000000 & 2.828427 & $-0.500000+0.500000 i$ & $0.000000+1.000000 i$ \\
\hline & & & 4.000000 & 2.828427 & $-0.500000+0.500000 i$ & $0.000000+1.000000 i$ \\
\hline & & & 4.000000 & 2.828427 & $-0.500000+0.500000 i$ & $0.000000+1.000000 i$ \\
\hline & & & 4.000000 & 2.828427 & $-0.500000+0.500000 i$ & $0.000000+1.000000 i$ \\
\hline \multirow{5}{*}{ L409001 } & satellite of $(2,2)$ torus & is link & & & & \\
\hline & 11.751836165335249 & 16 & 3.387794 & 1.790834 & $-0.145301+0.423474 i$ & $0.060606+0.465438 i$ \\
\hline & & & 3.387794 & 1.790834 & $-0.145301+0.423474 i$ & $0.060606+0.465438 i$ \\
\hline & & & 3.387794 & 1.790834 & $-0.145301+0.423474 i$ & $0.060606+0.465438 i$ \\
\hline & & & 3.387794 & 1.790834 & $-0.145301+0.423474 i$ & $0.060606+0.465438$ \\
\hline
\end{tabular}

TABLE 1 (continued) 


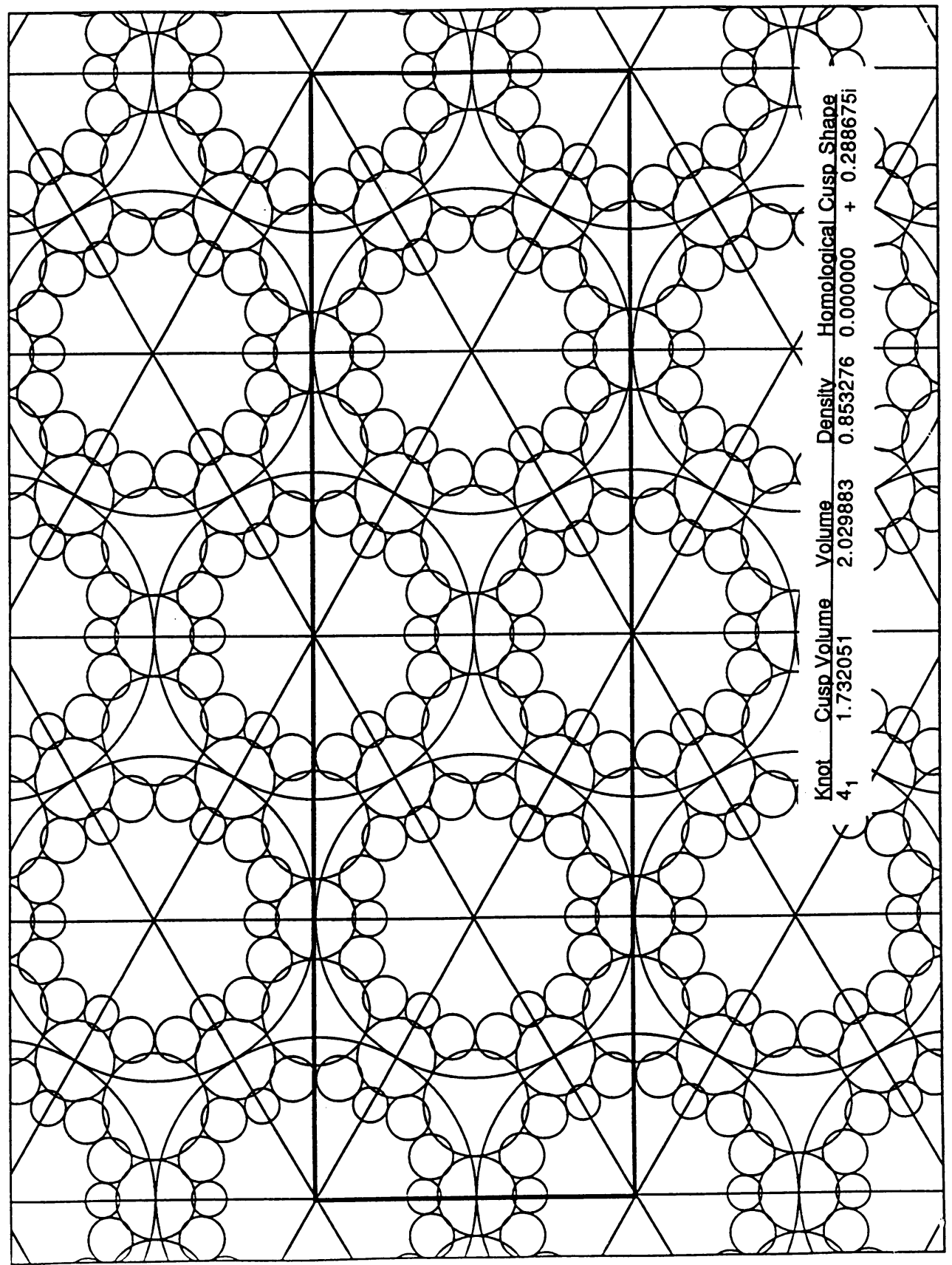

TABLE 2 


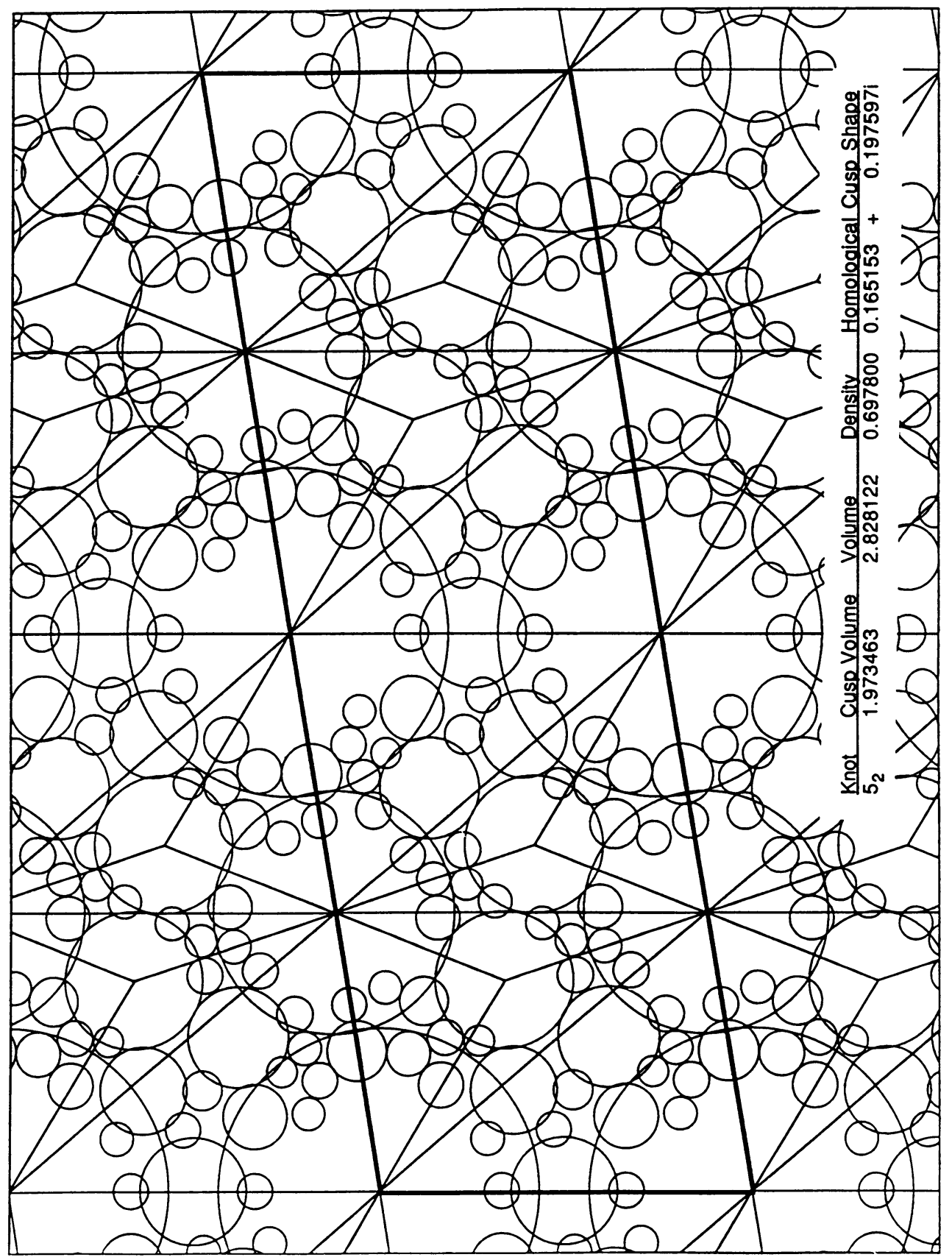

TABLE 2 (continued) 


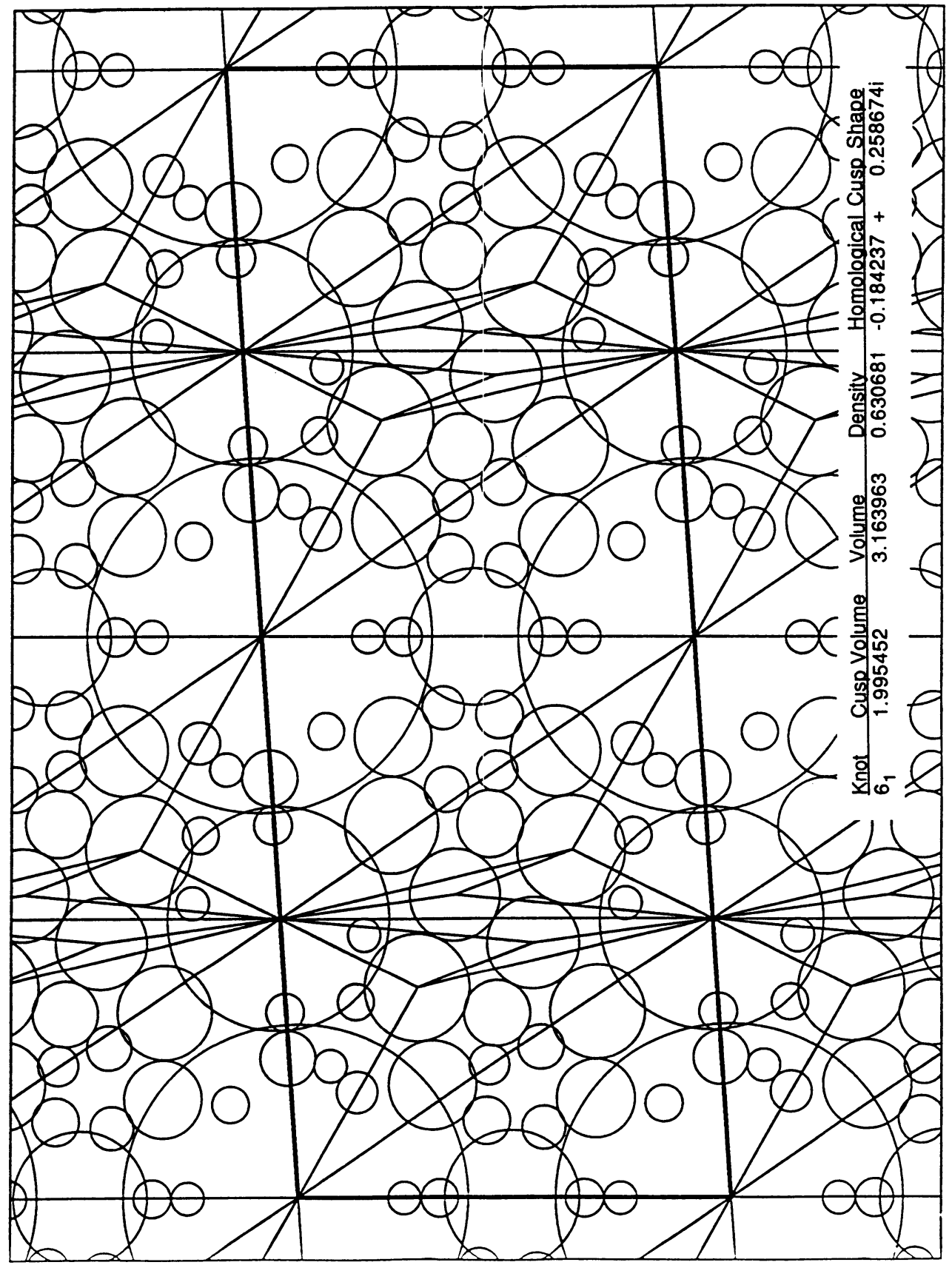

TABLE 2 (continued) 


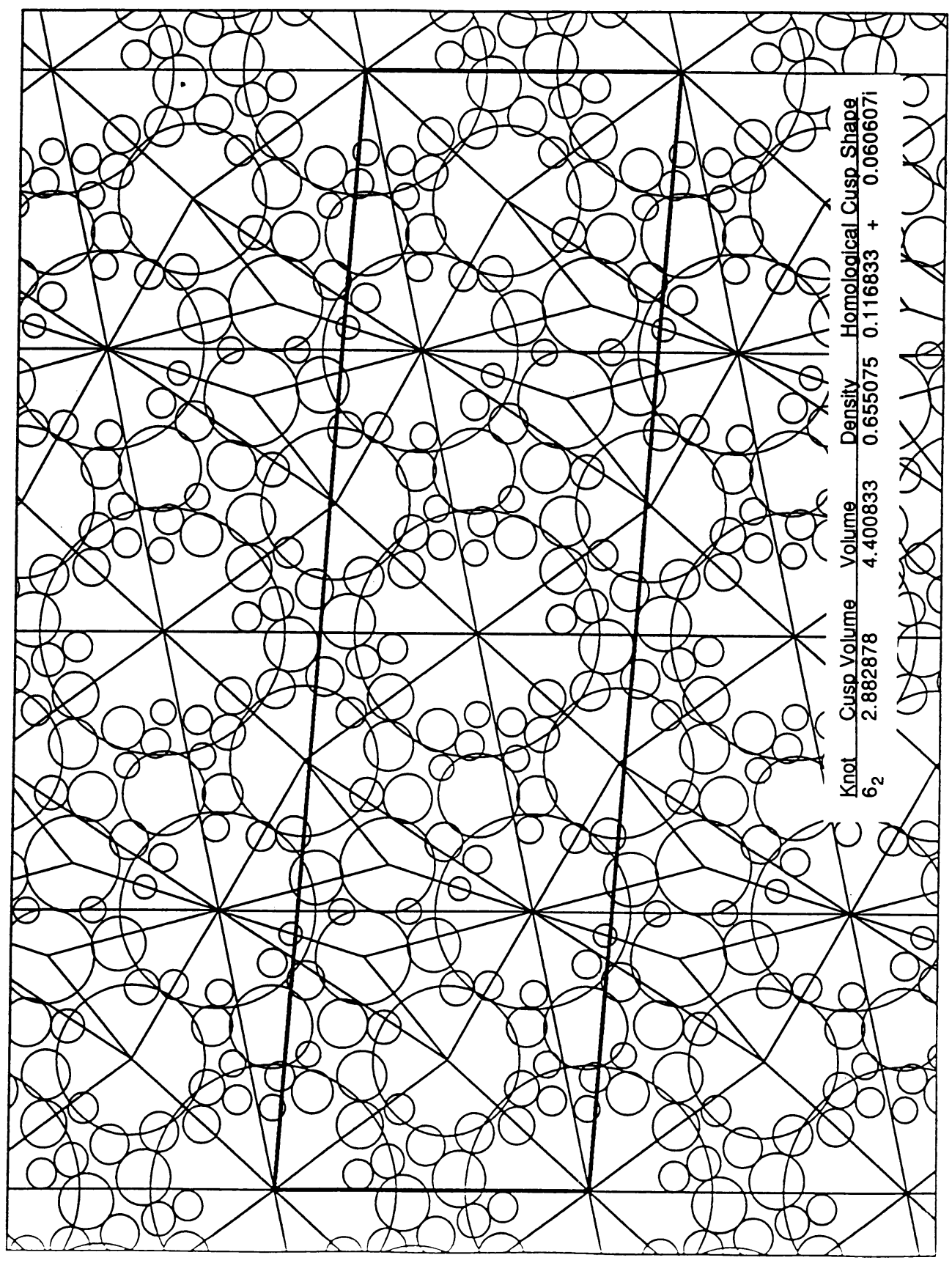

TABLE 2 (continued) 


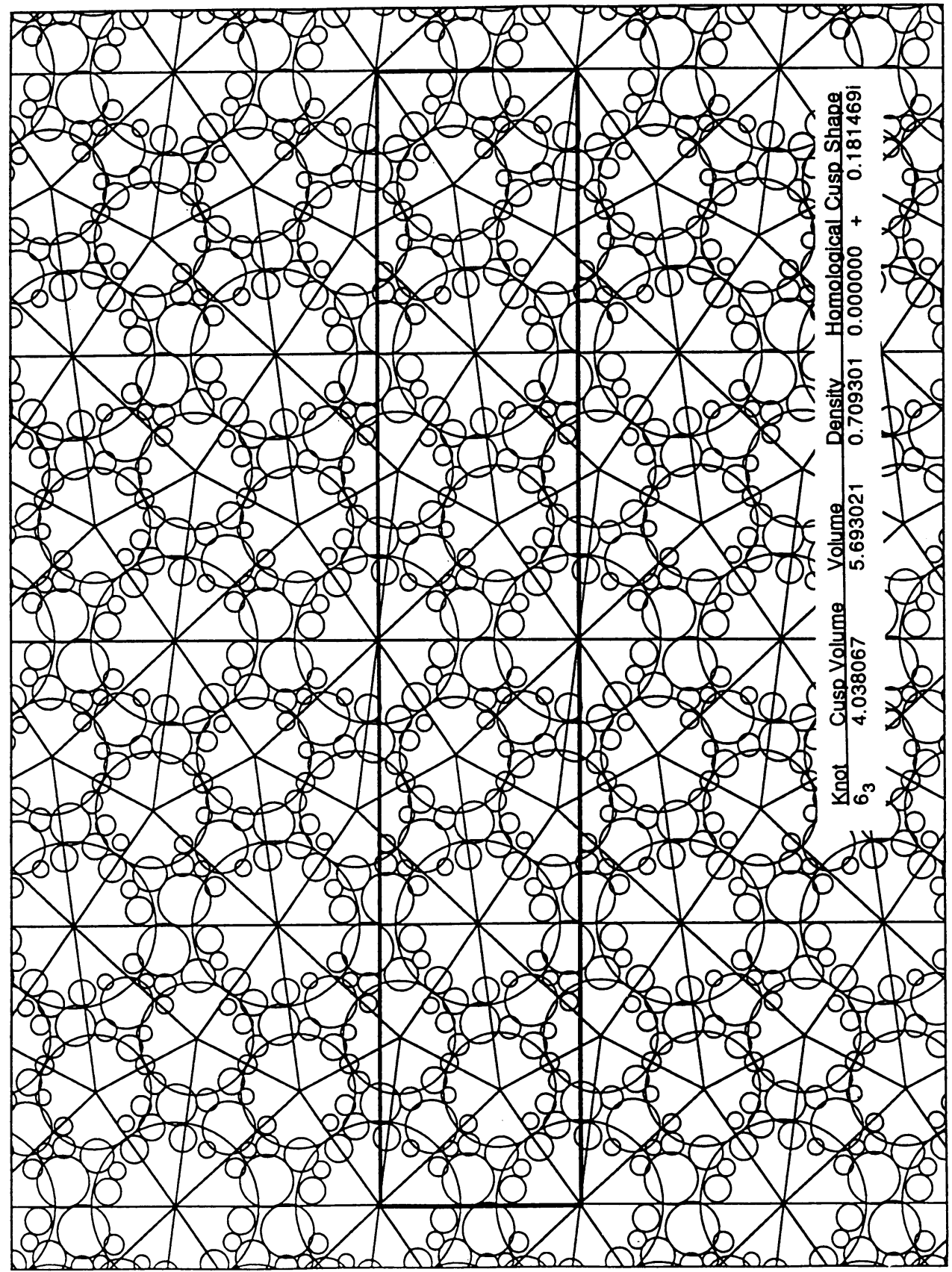

TABLE 2 (continued) 


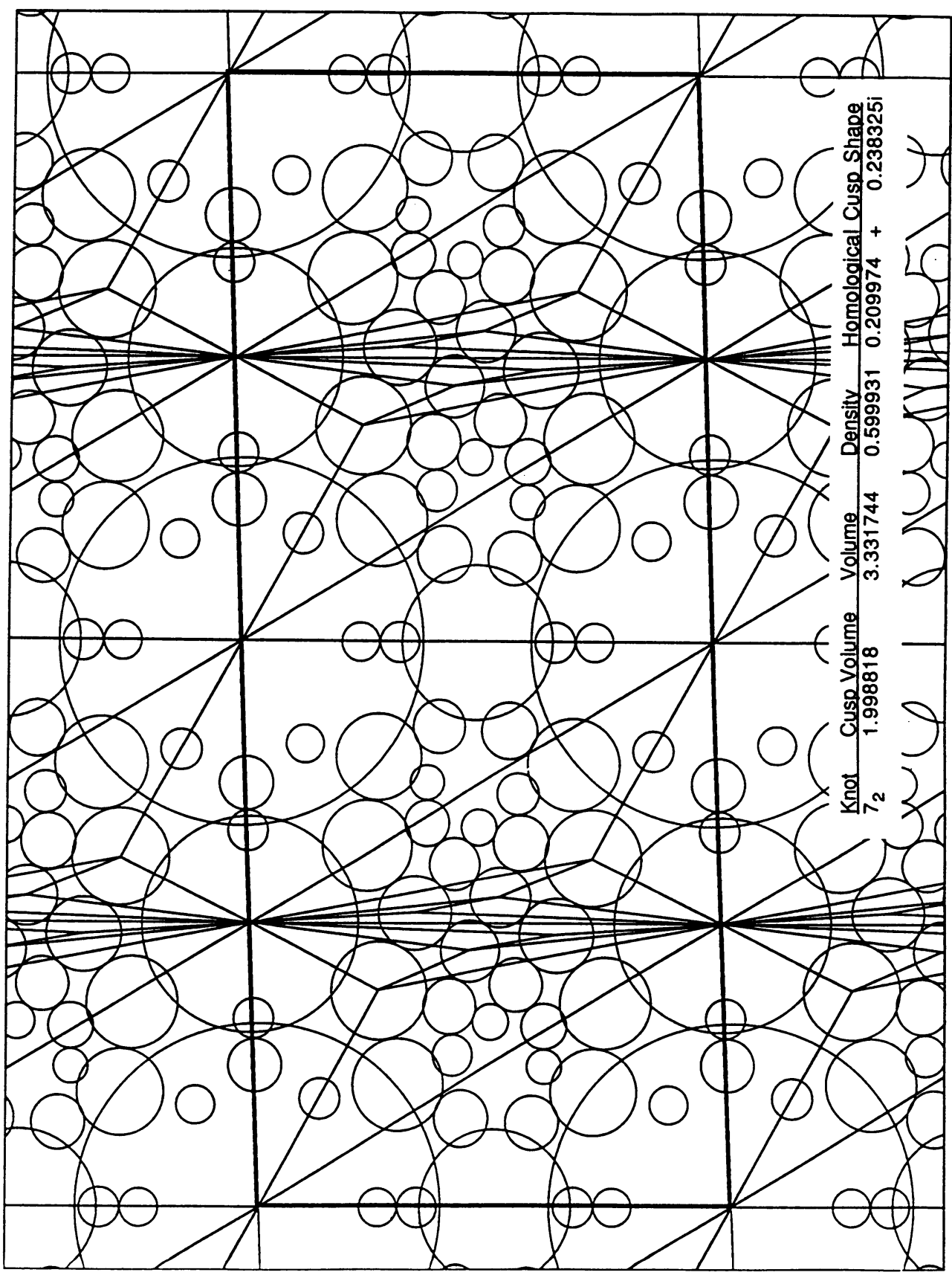

TABLE 2 (continued) 


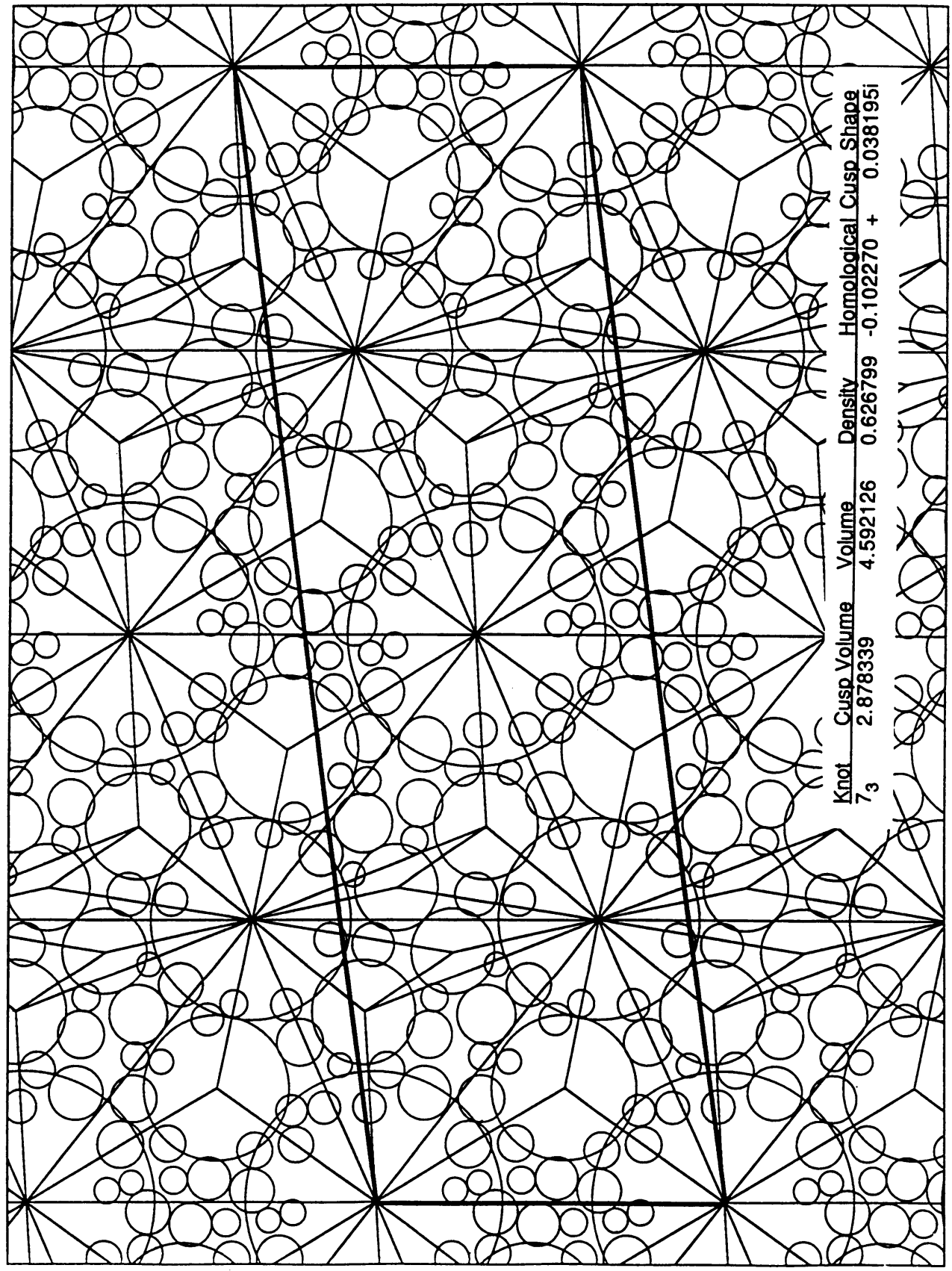

TABLE 2 (continued) 


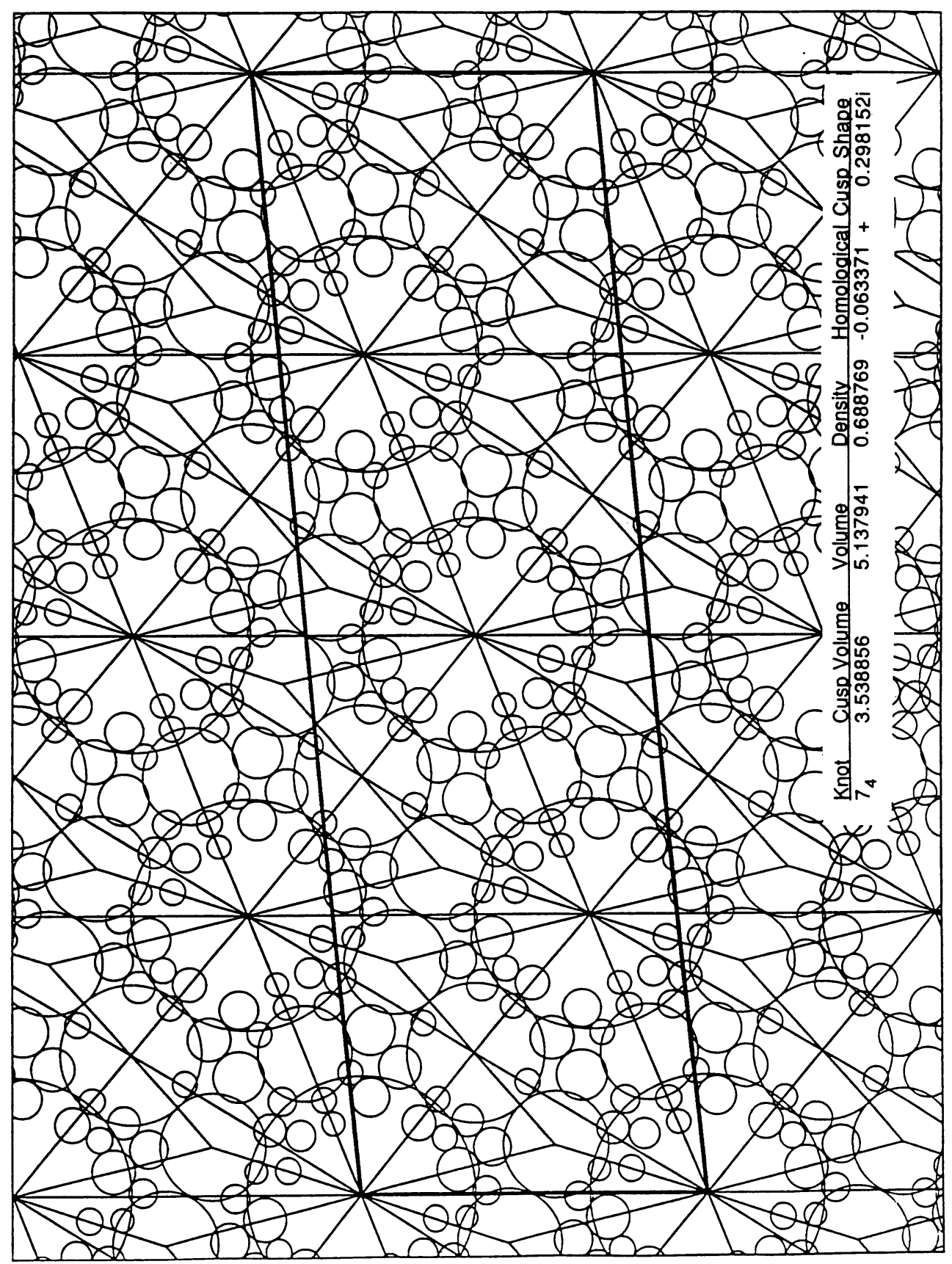

TABLE 2 (continued) 


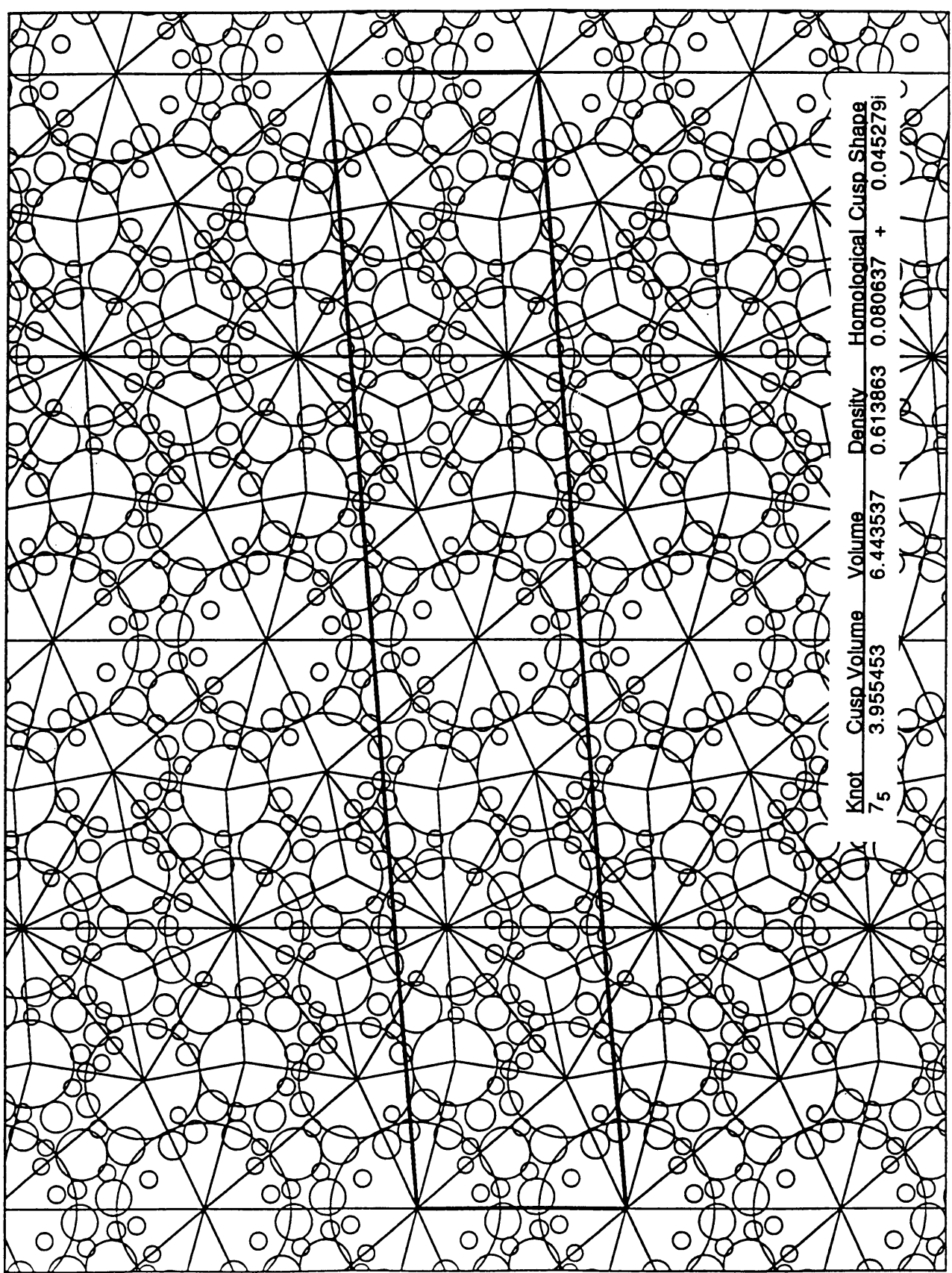

TABLE 2 (continued) 


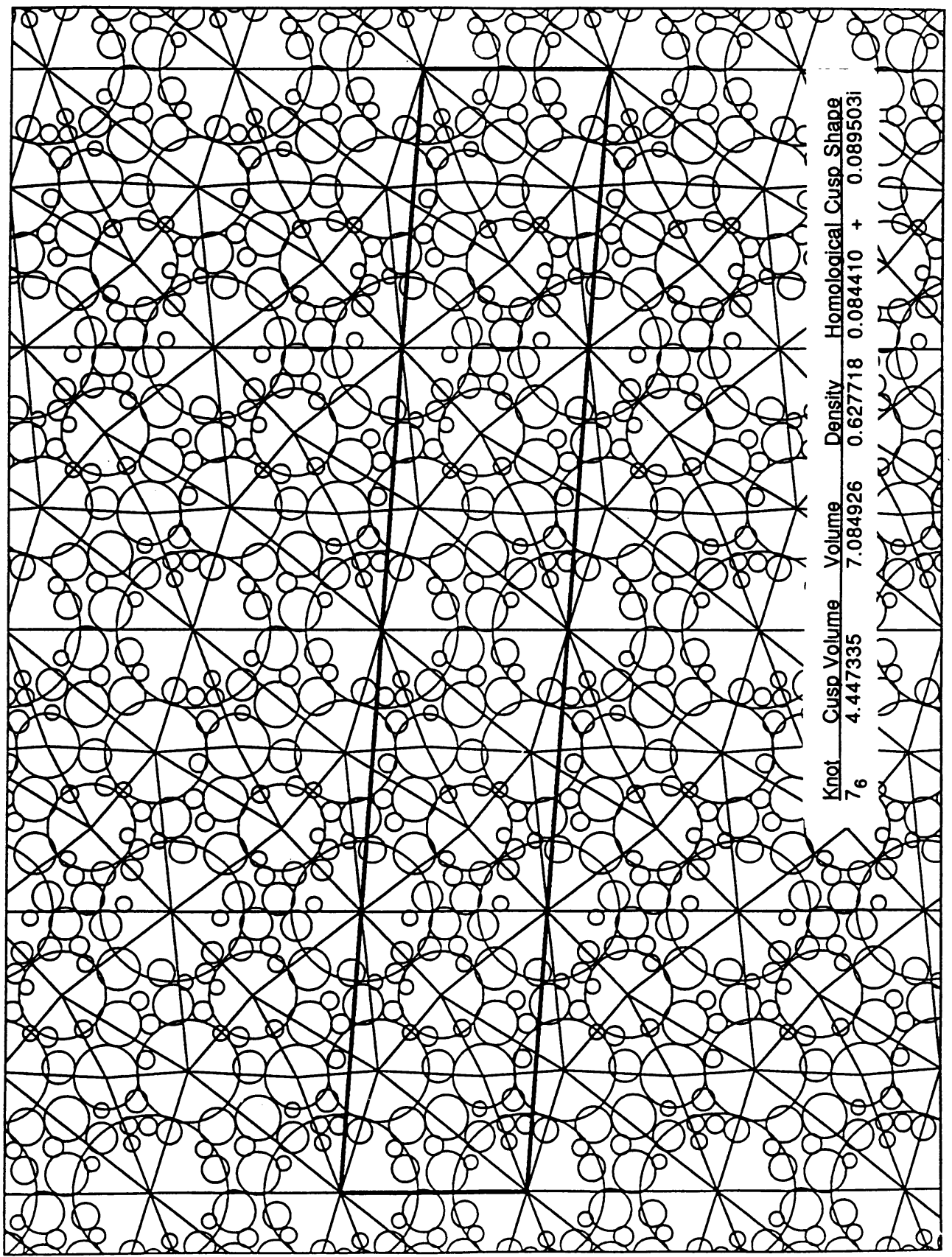

TABLE 2 (continued) 


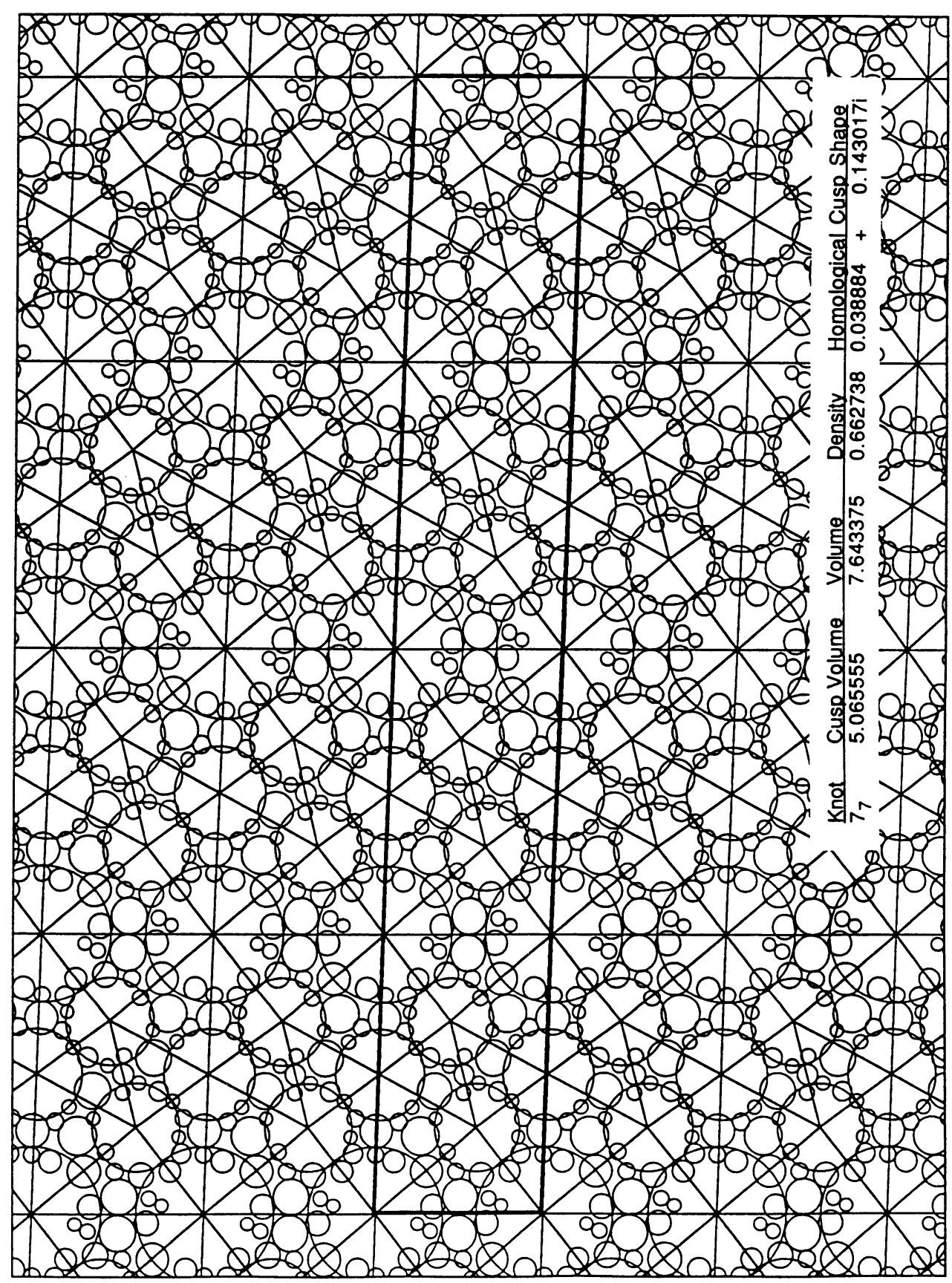

TABLE 2 (continued) 


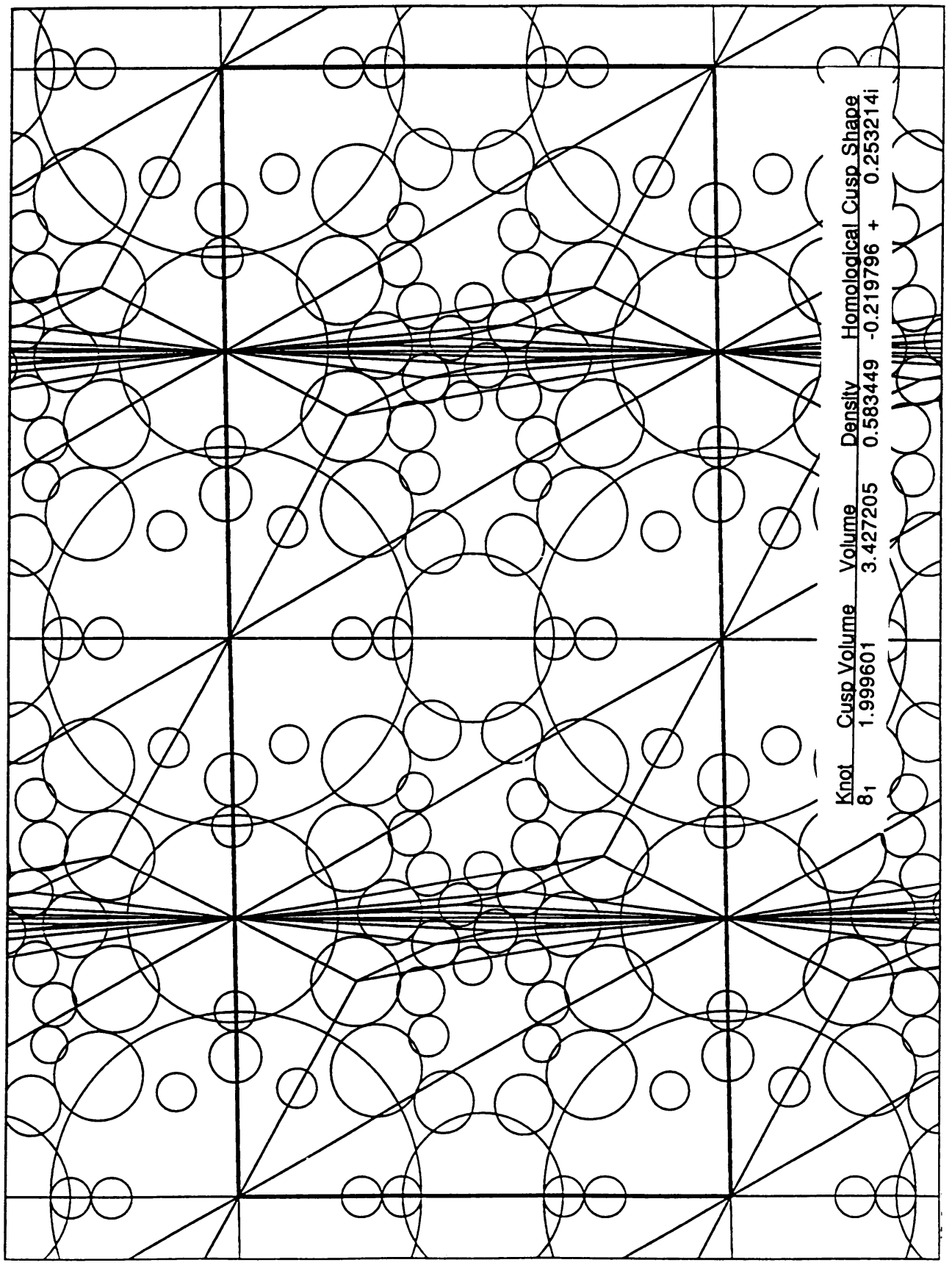

TABLE 2 (continued) 


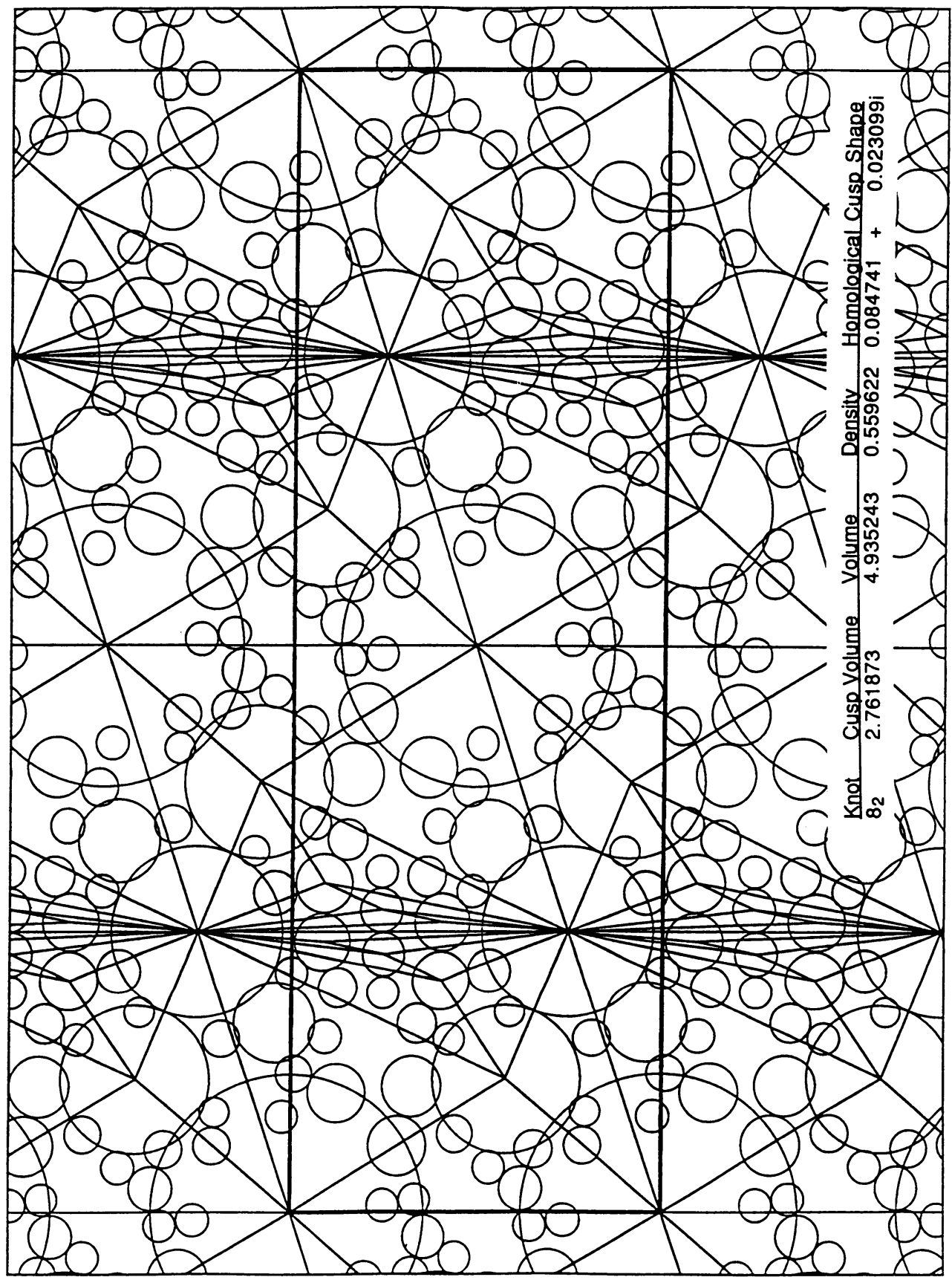

TABLE 2 (continued) 


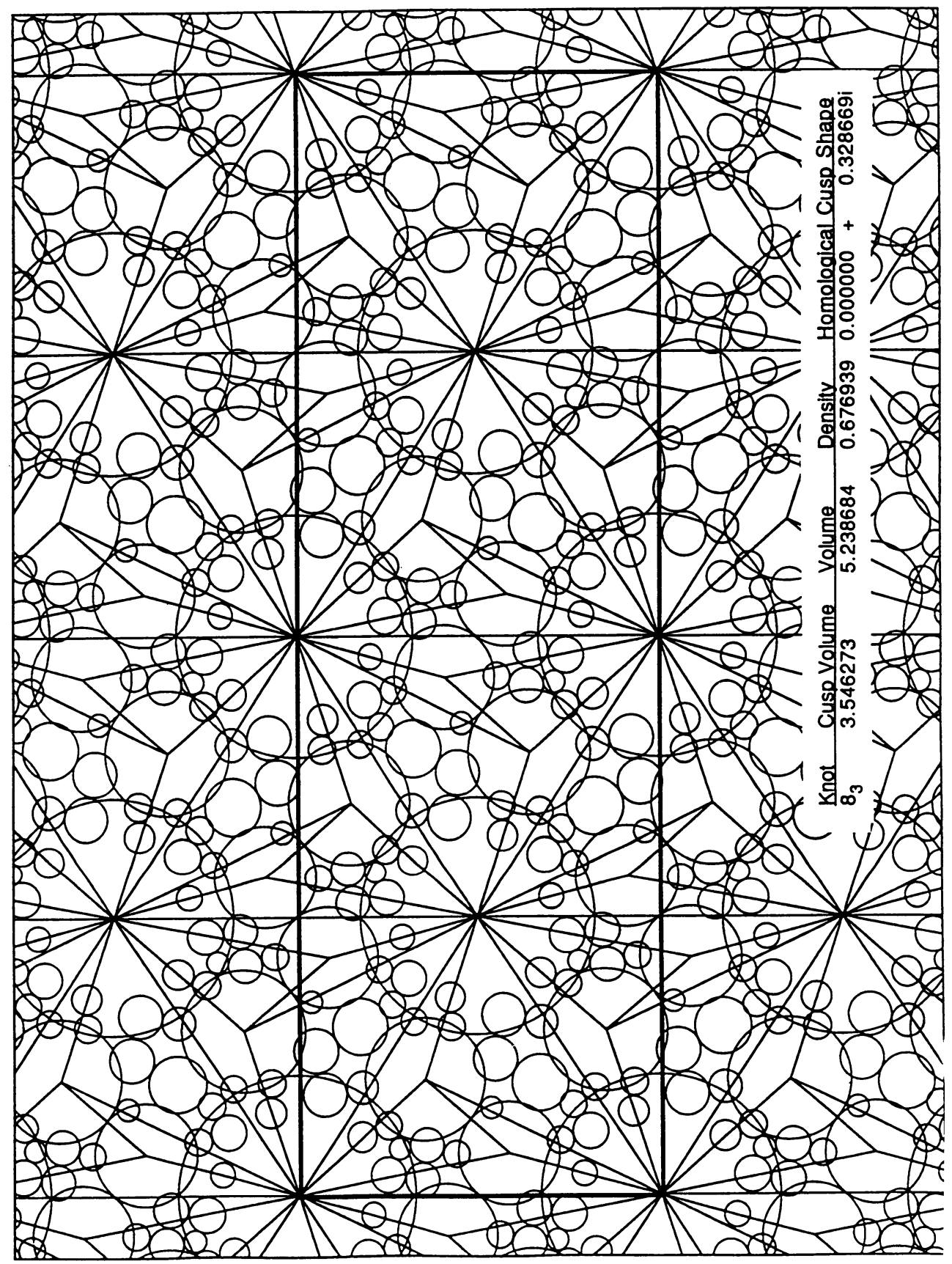

TABLE 2 (continued) 


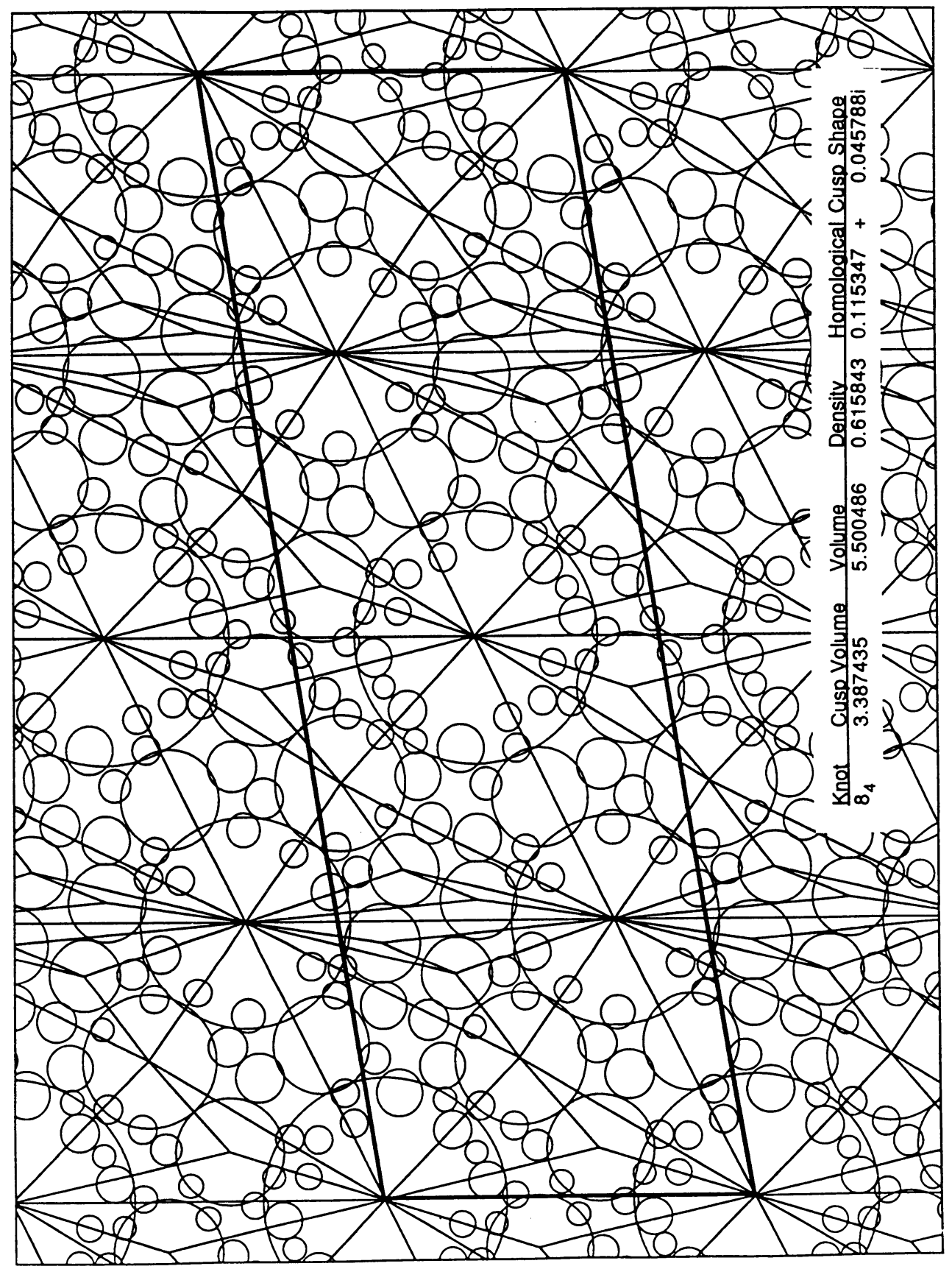

TABLE 2 (continued) 


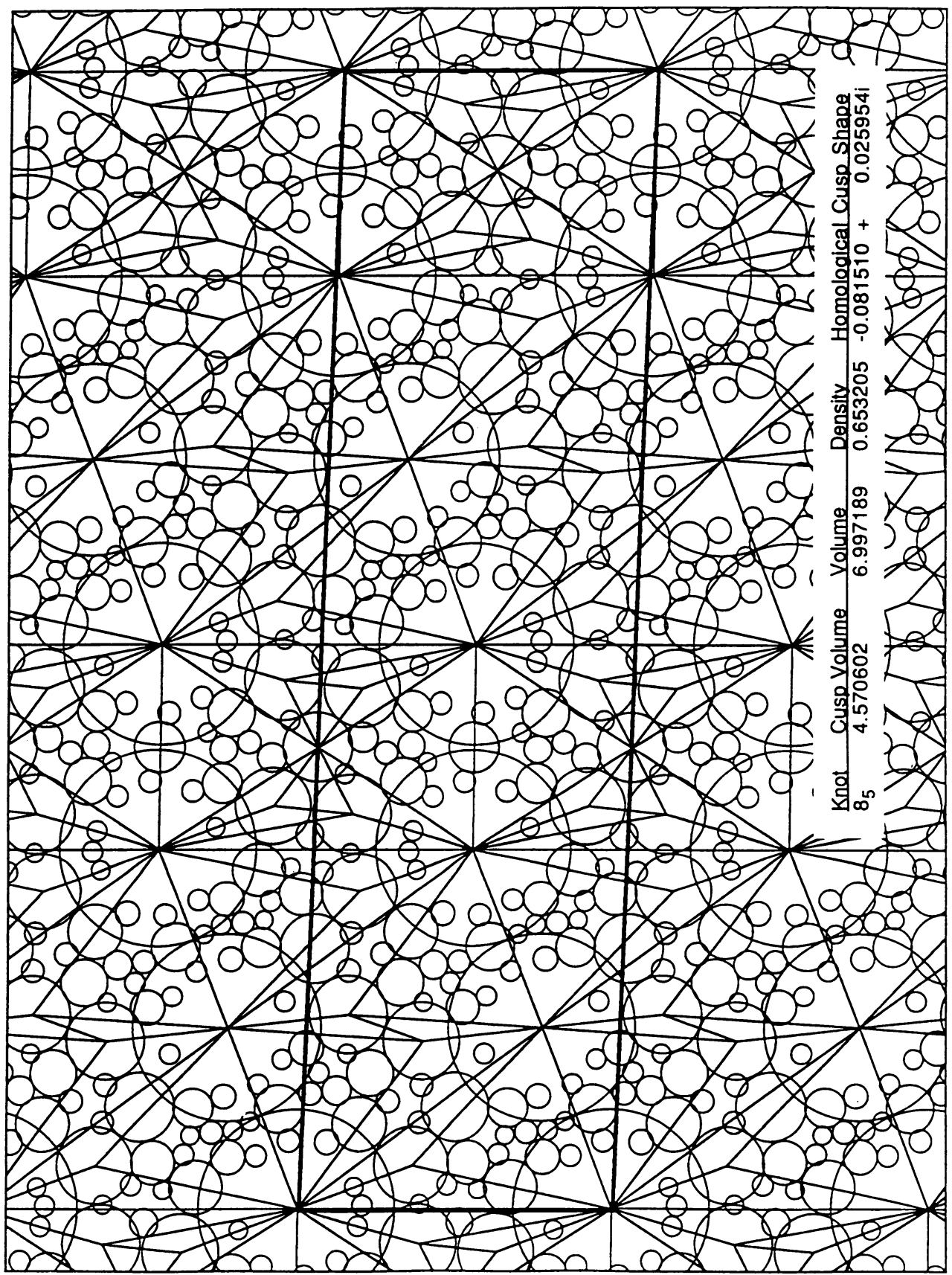

TABLE 2 (continued) 


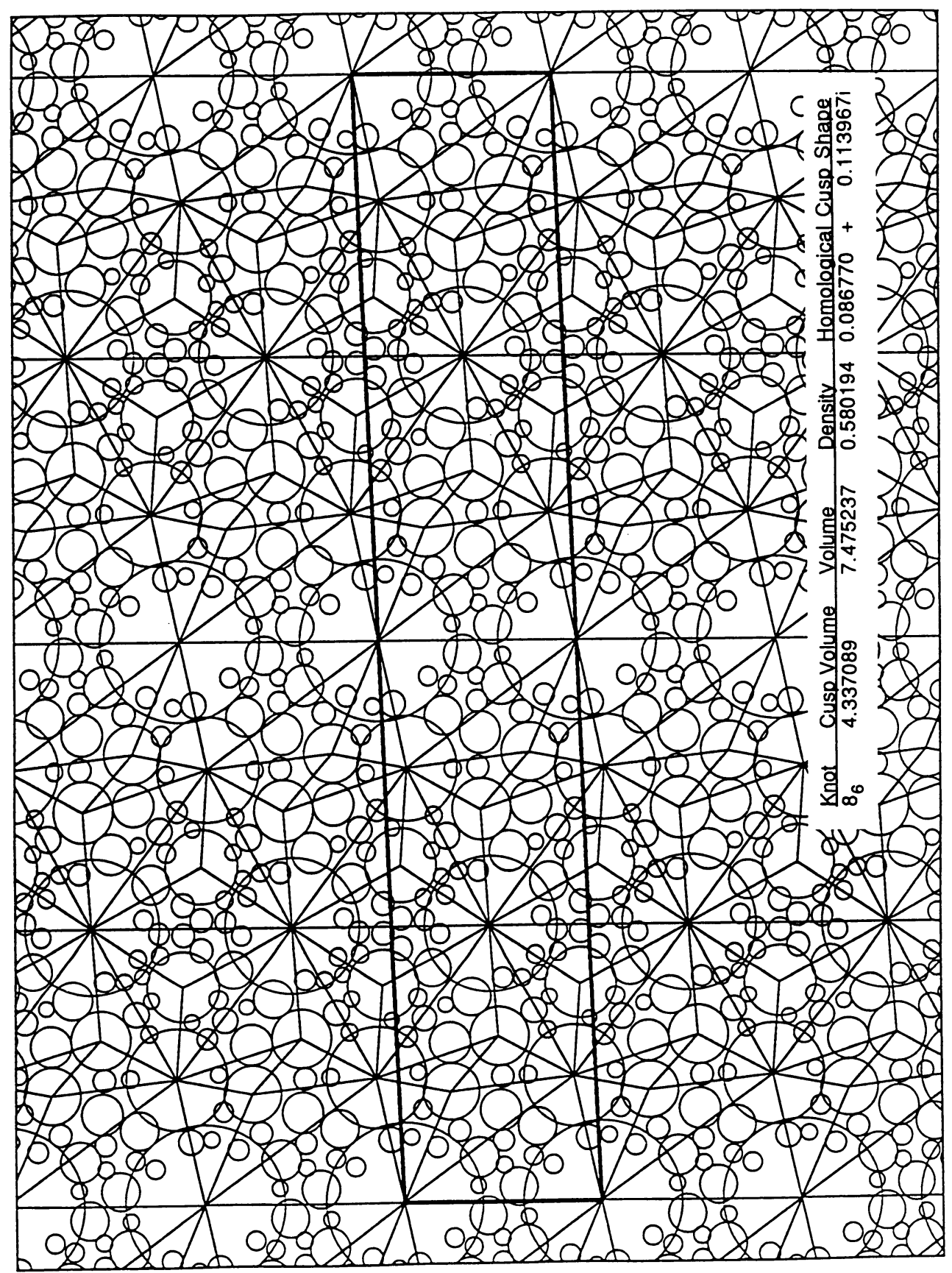

TABLE 2 (continued) 


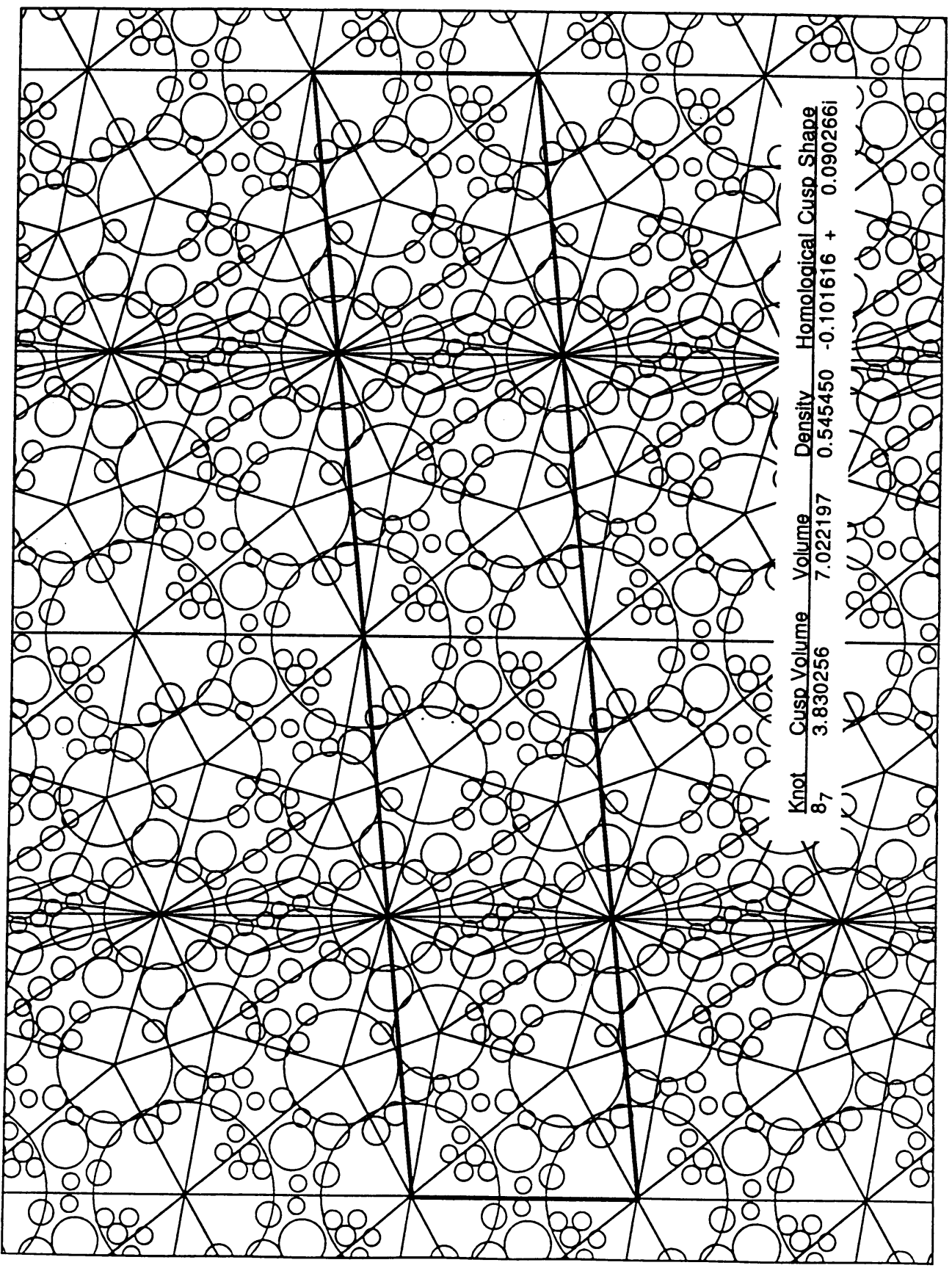

TABLE 2 (continued) 


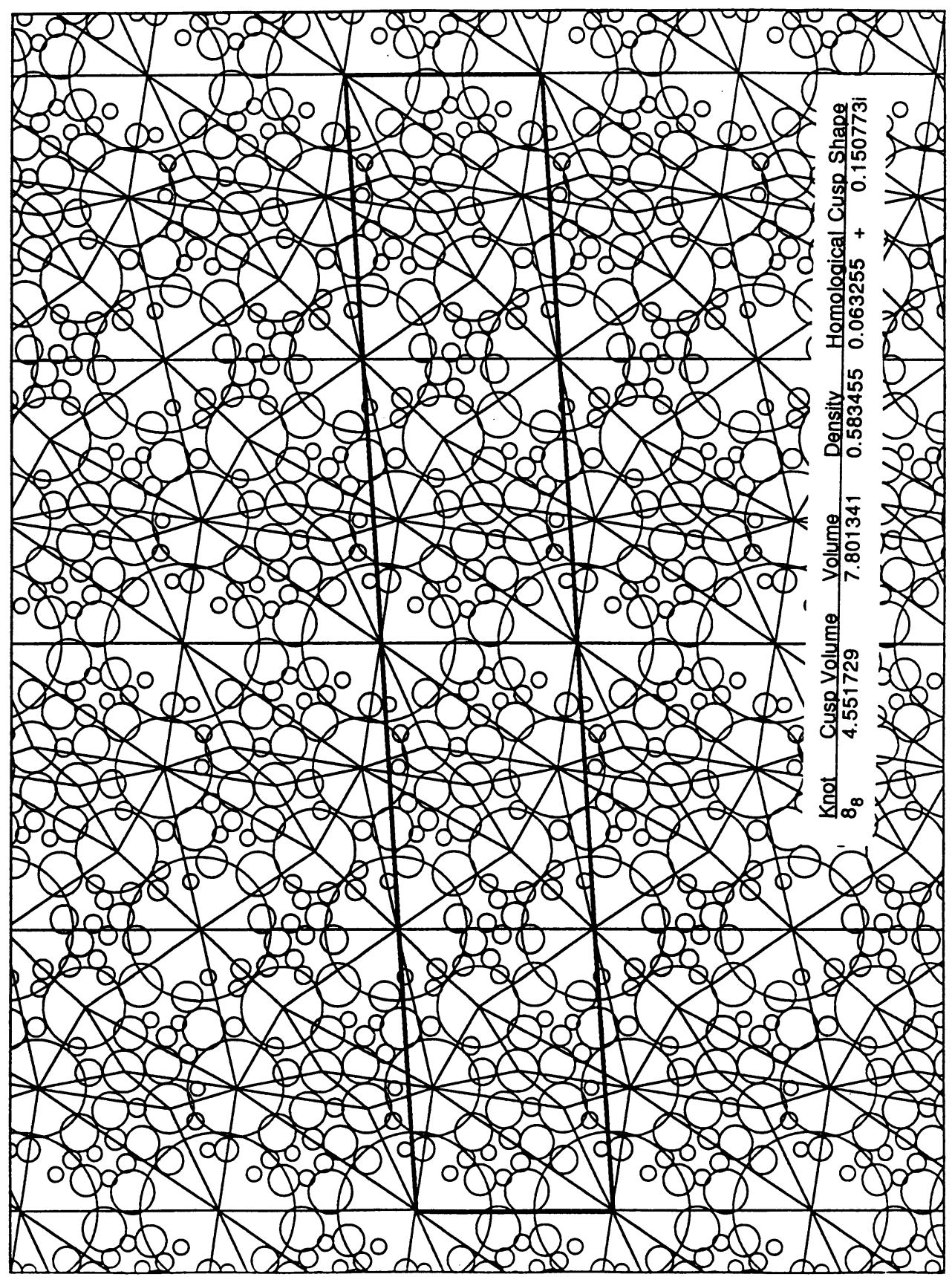

TABLE 2 (continued) 


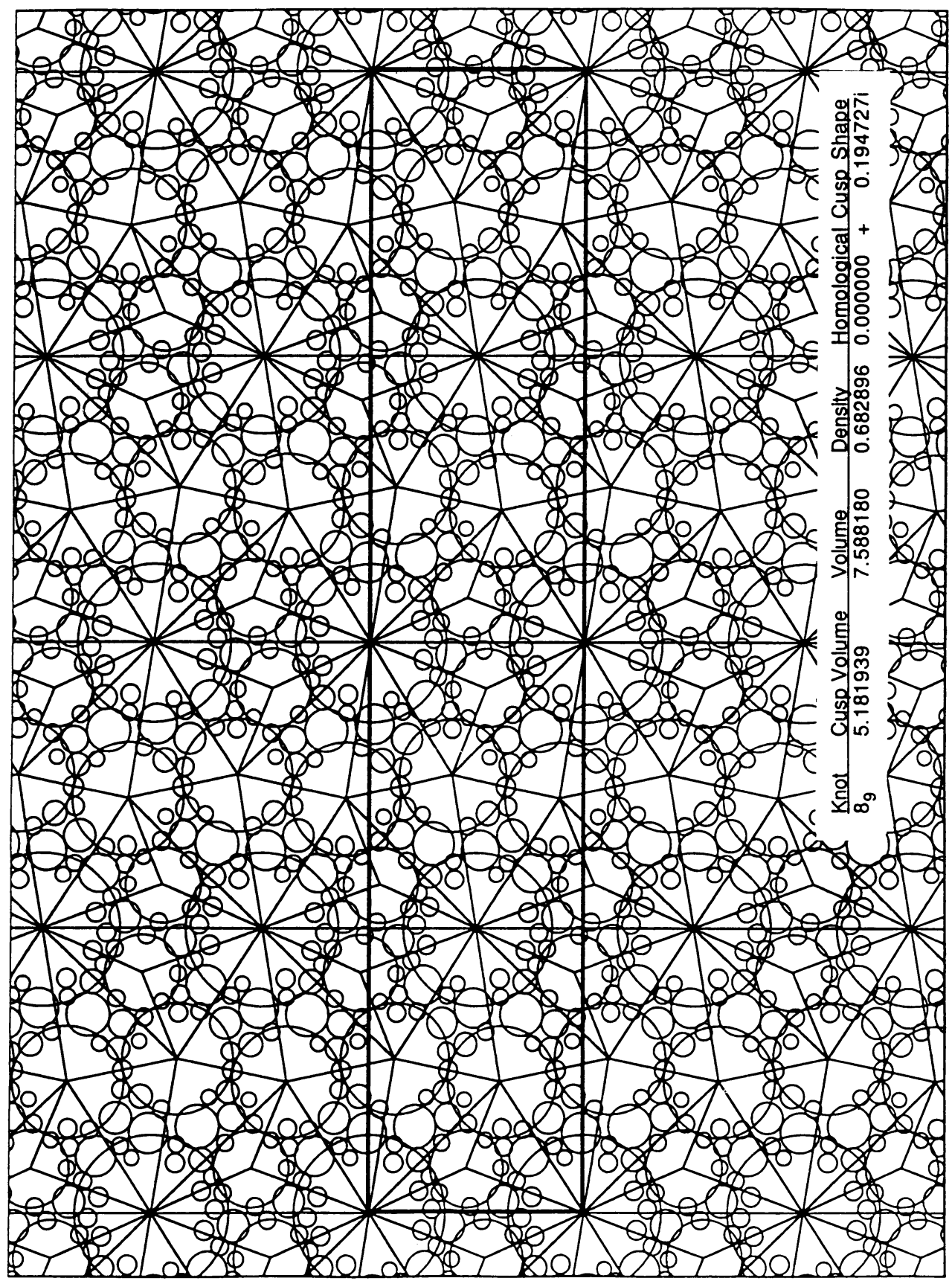

TABLE 2 (continued) 


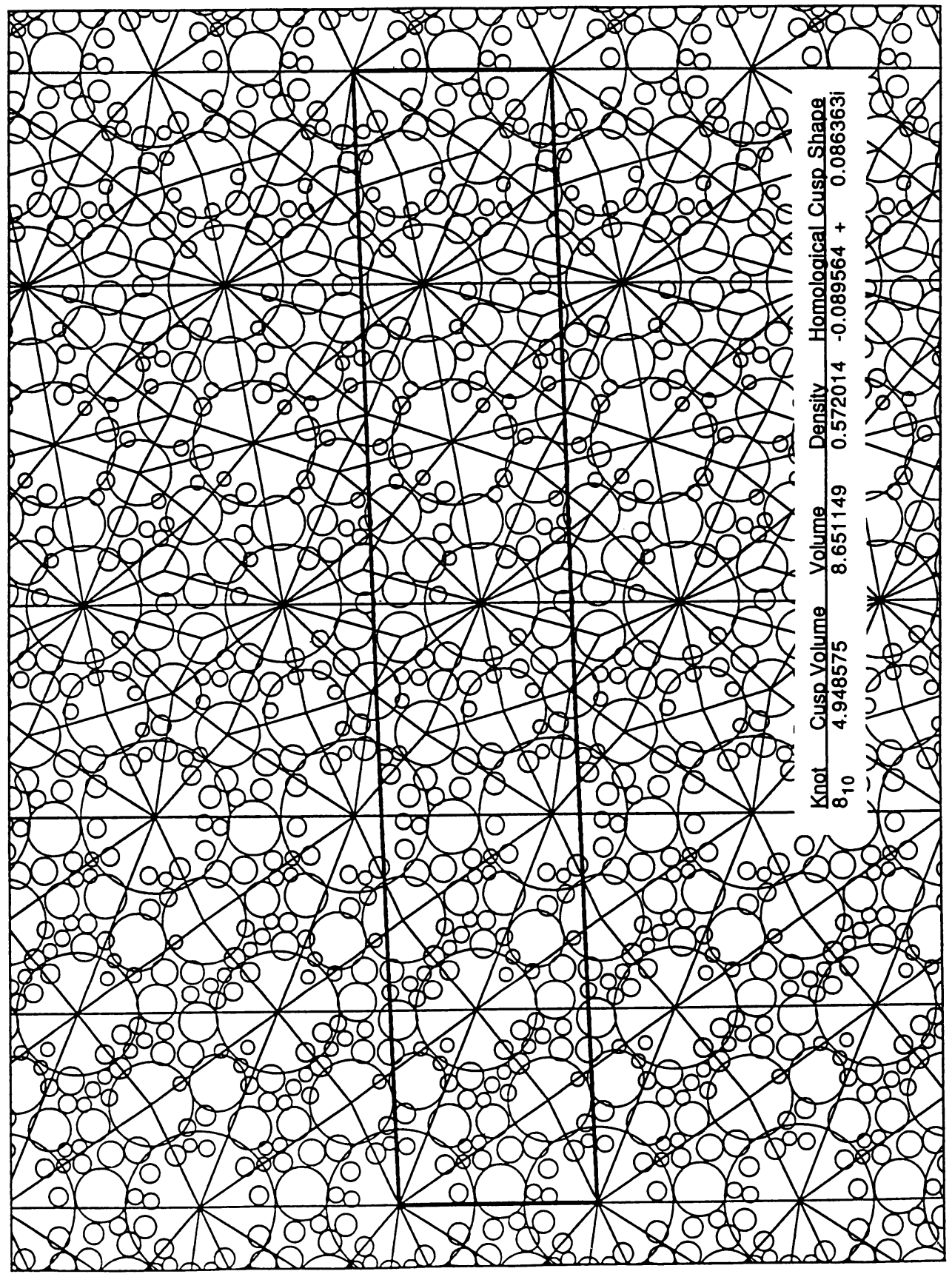

TABLE 2 (continued) 


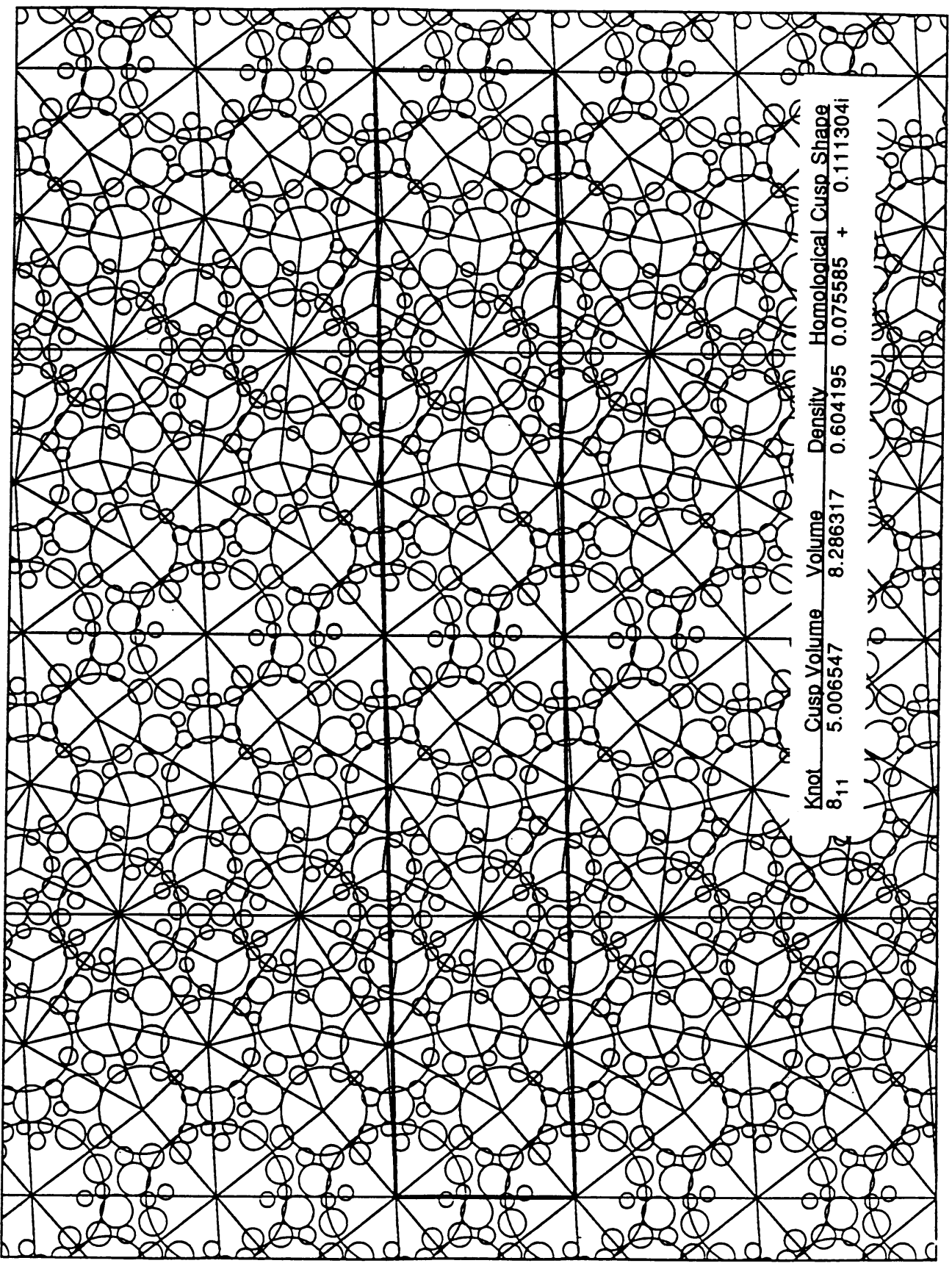

TABLE 2 (continued) 


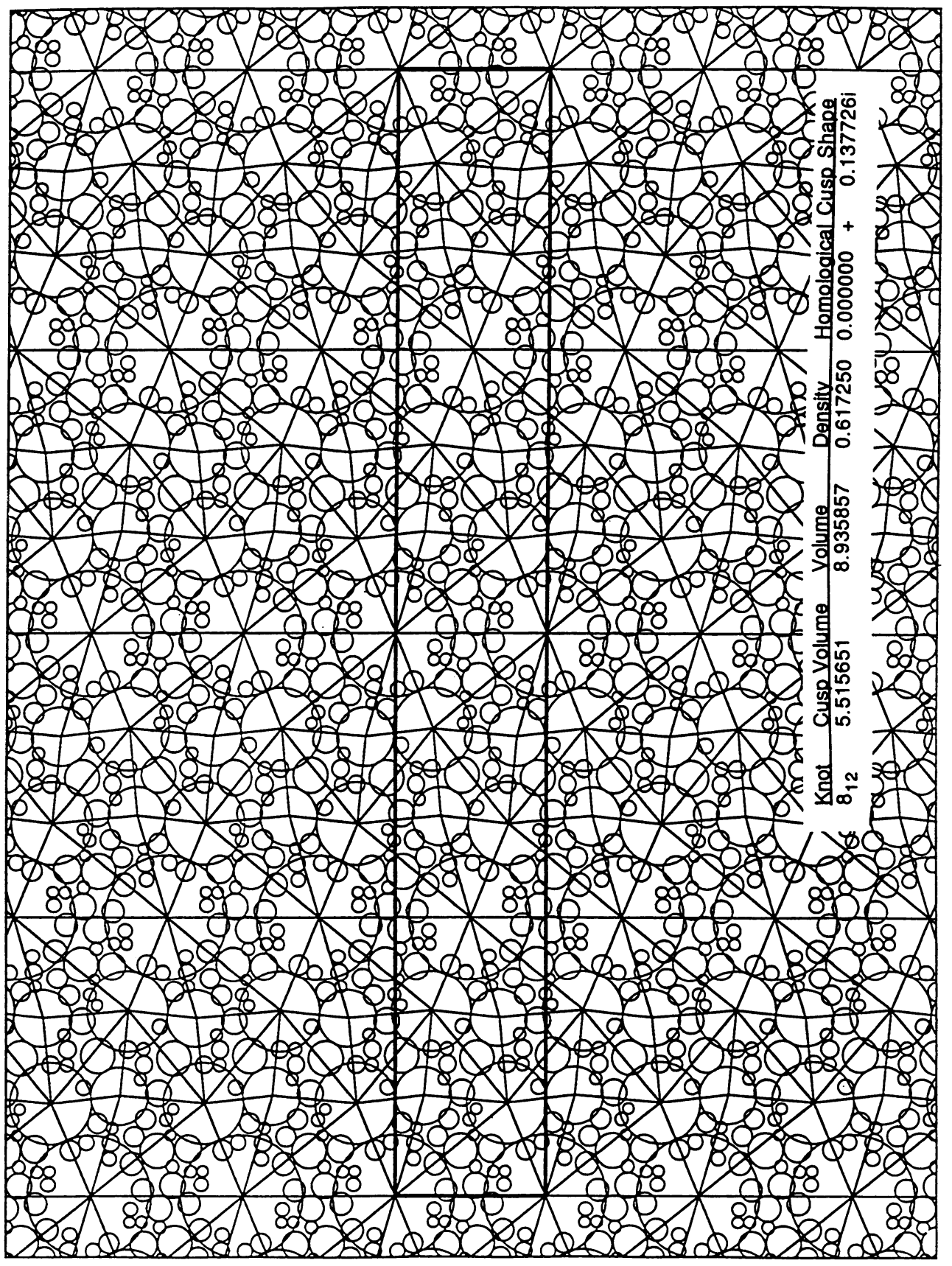

TABLE 2 (continued) 


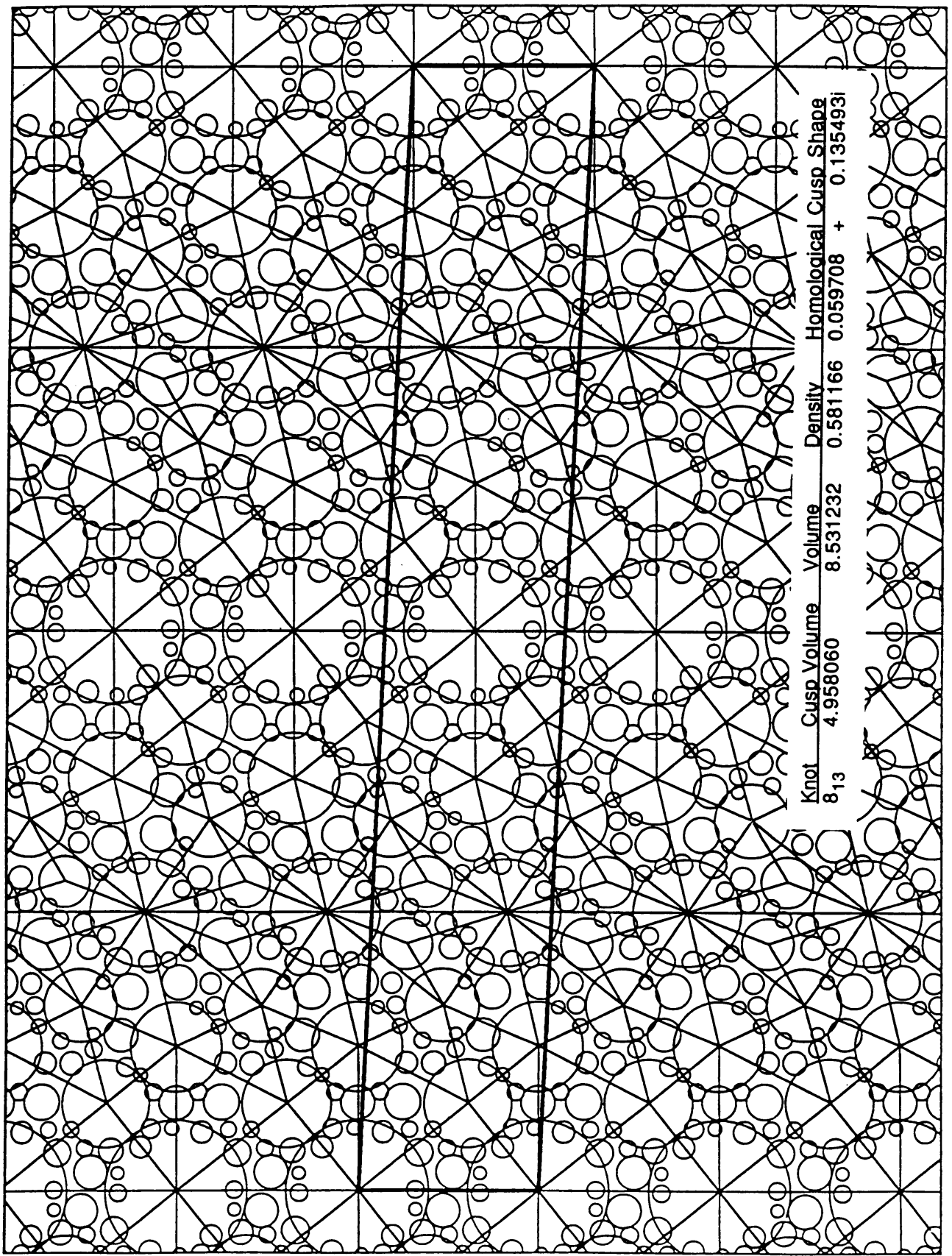

TABLE 2 (continued) 


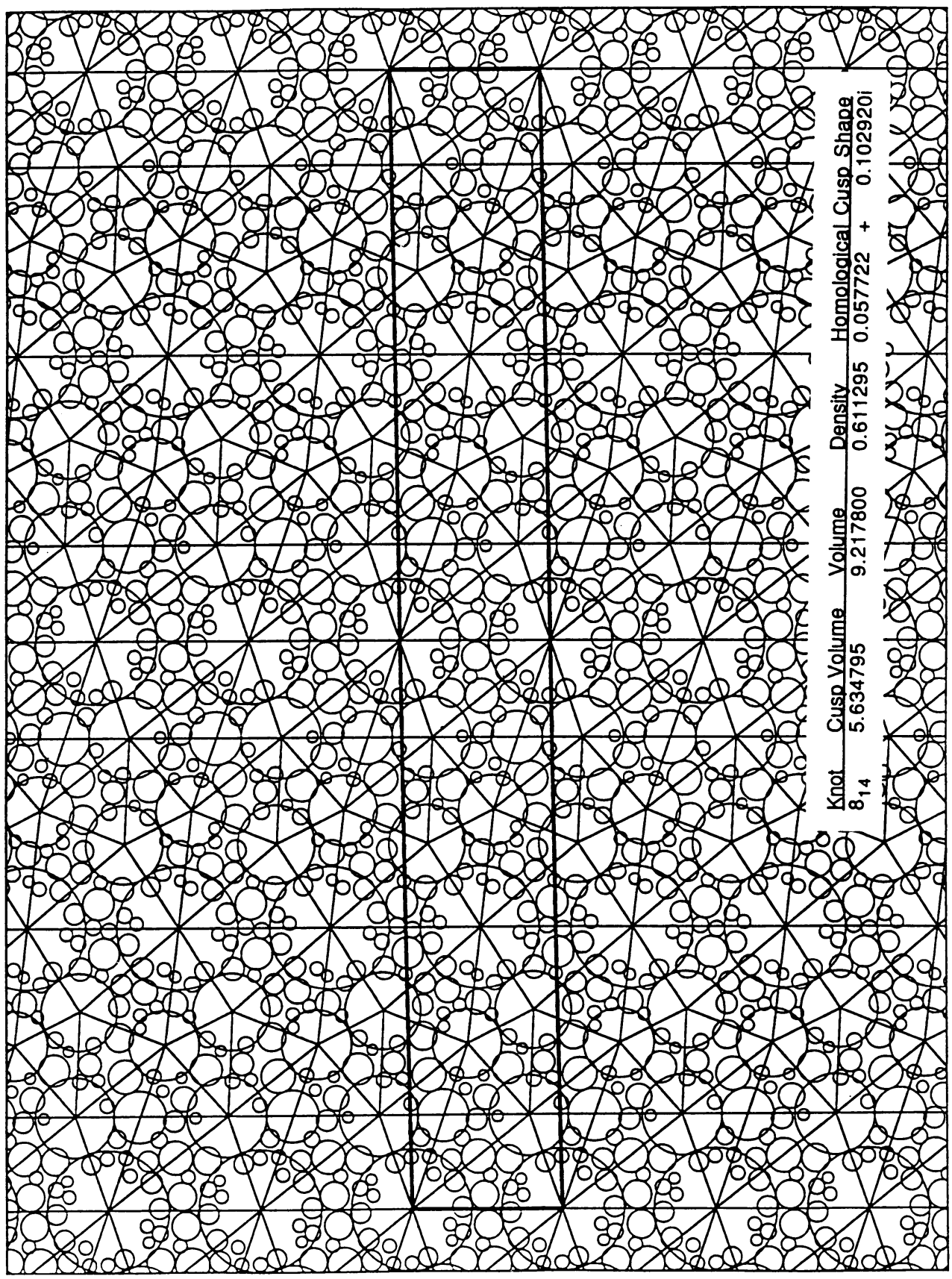

TABLE 2 (continued) 


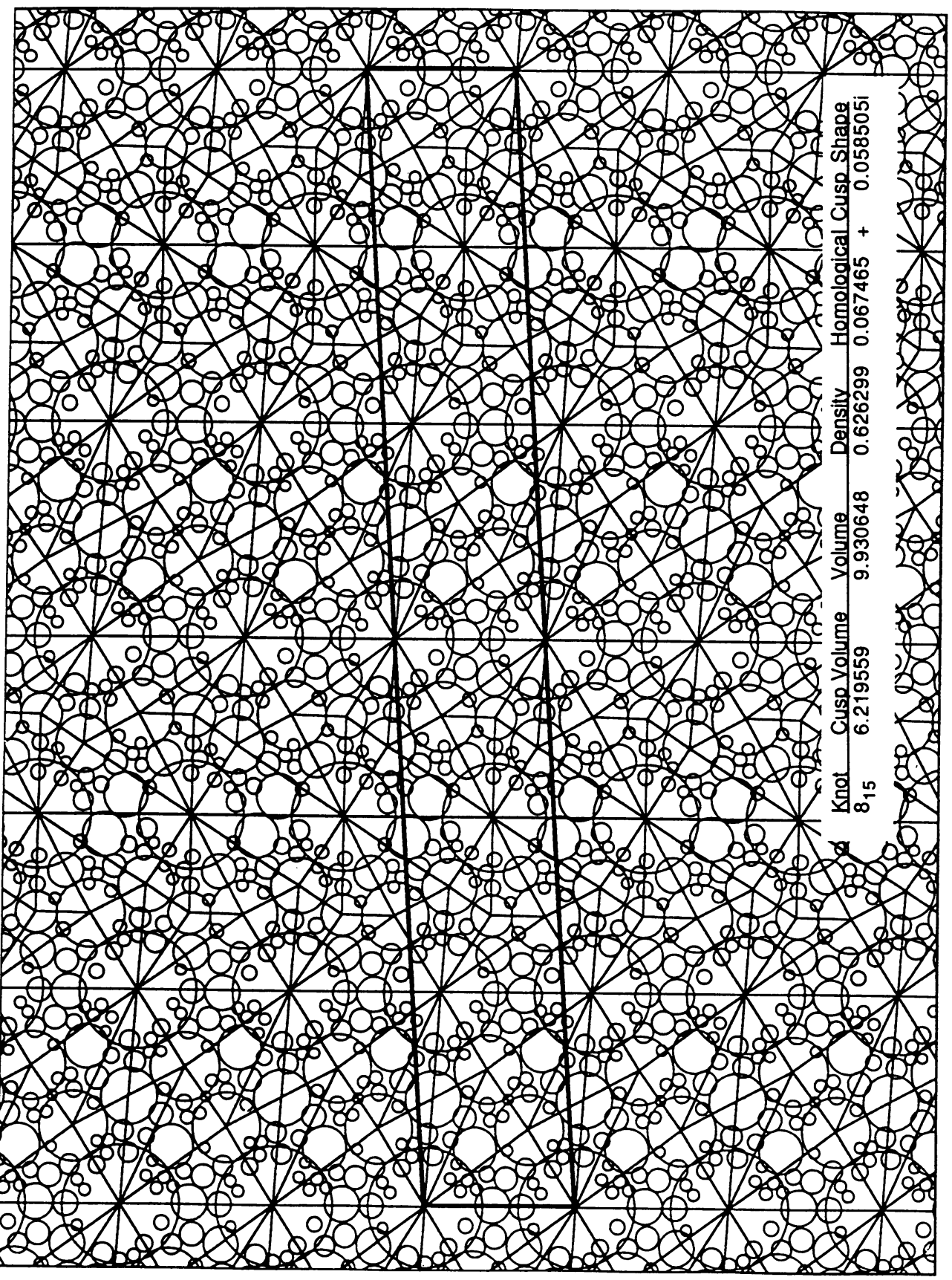

TABLE 2 (continued) 


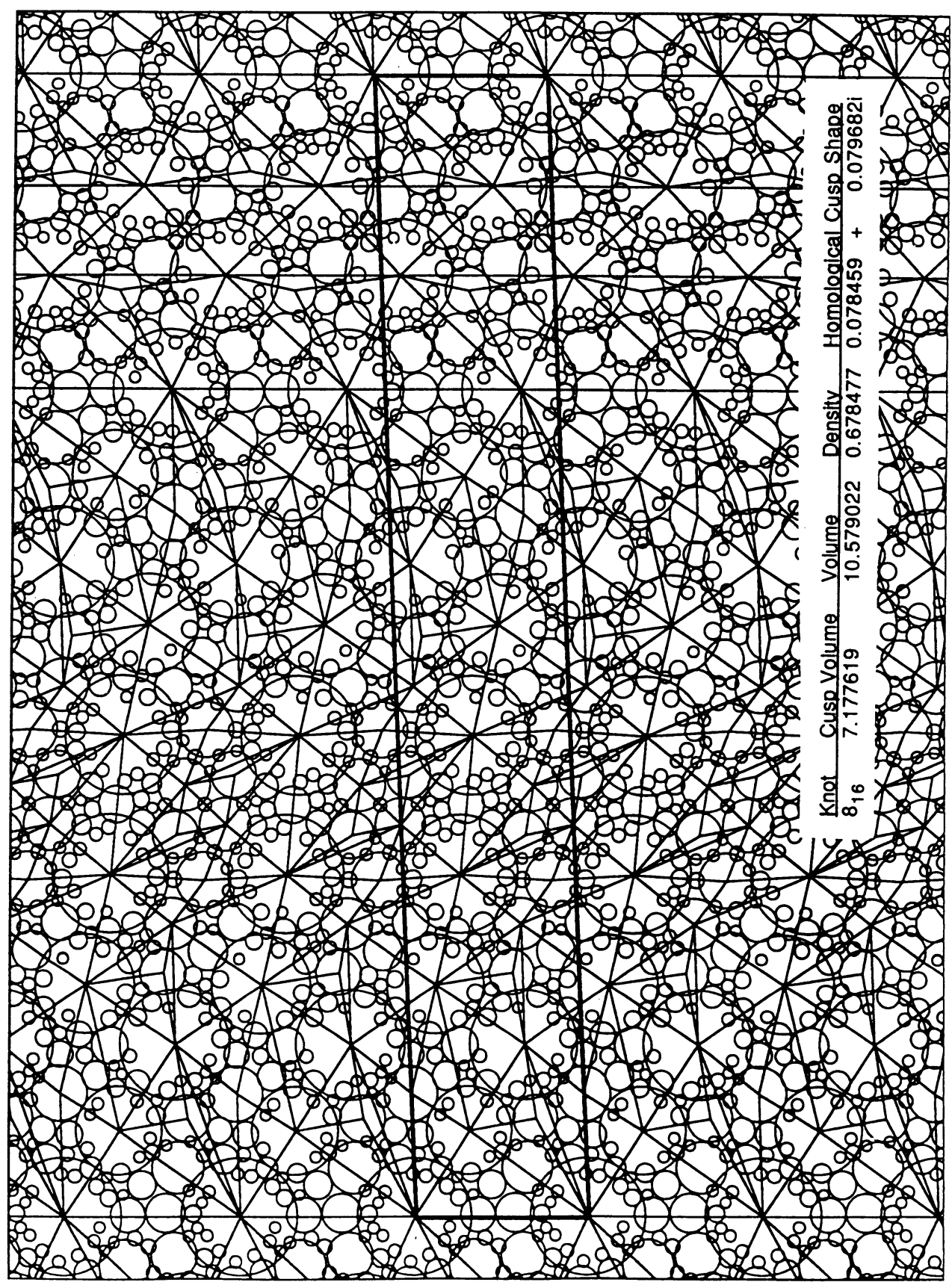

TABLE 2 (continued) 


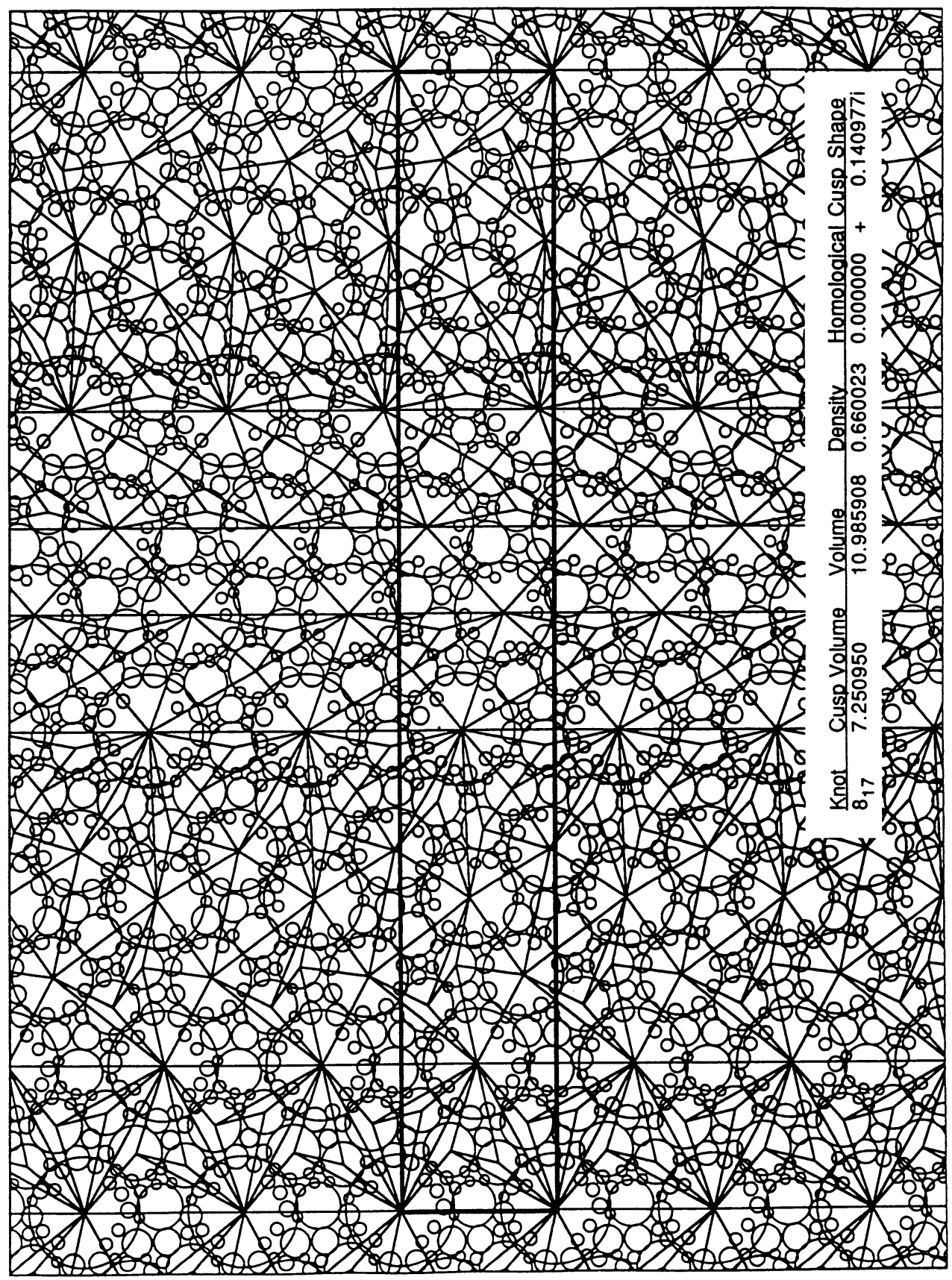

TABLE 2 (continued) 


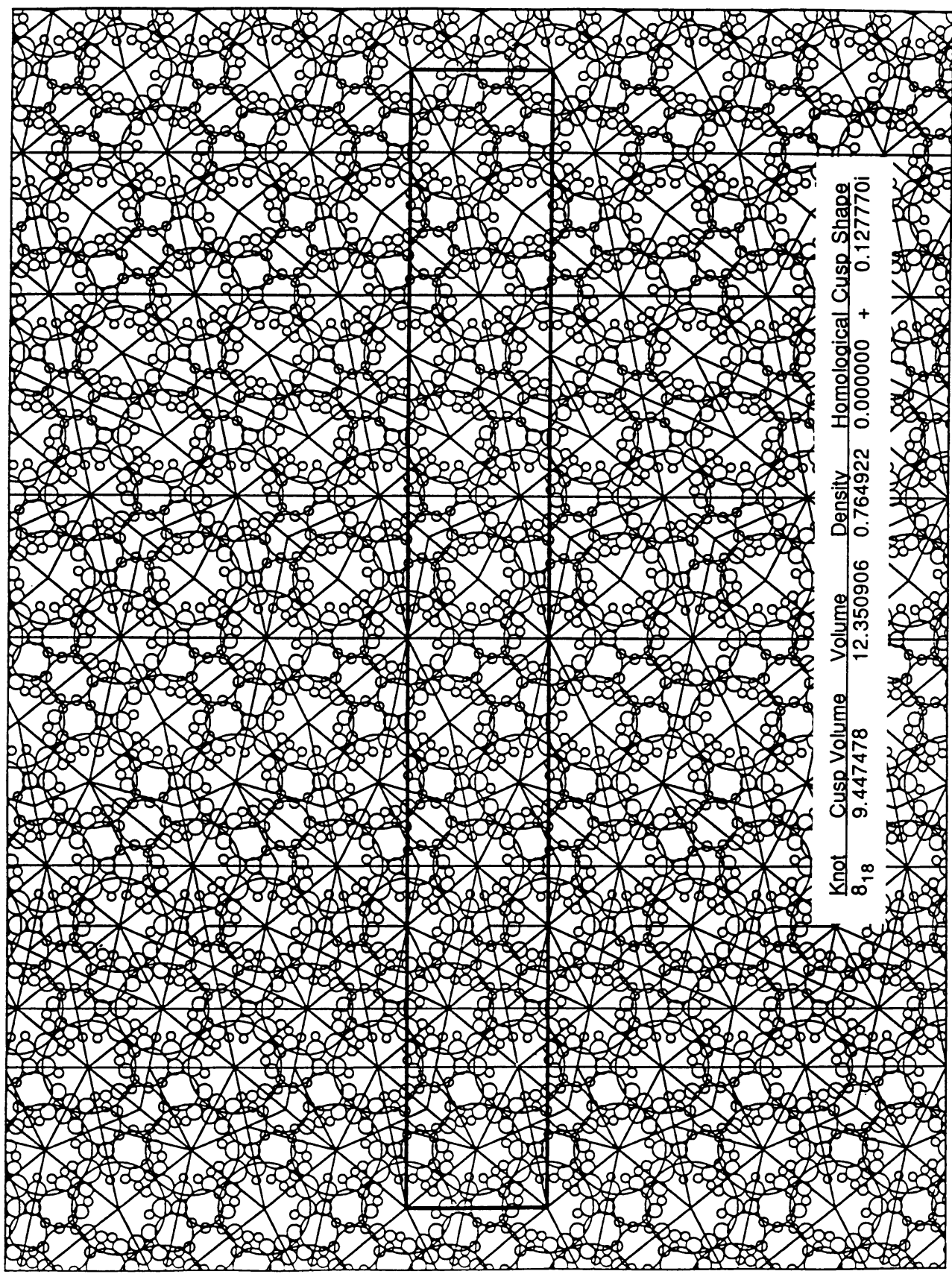

TABLE 2 (continued) 


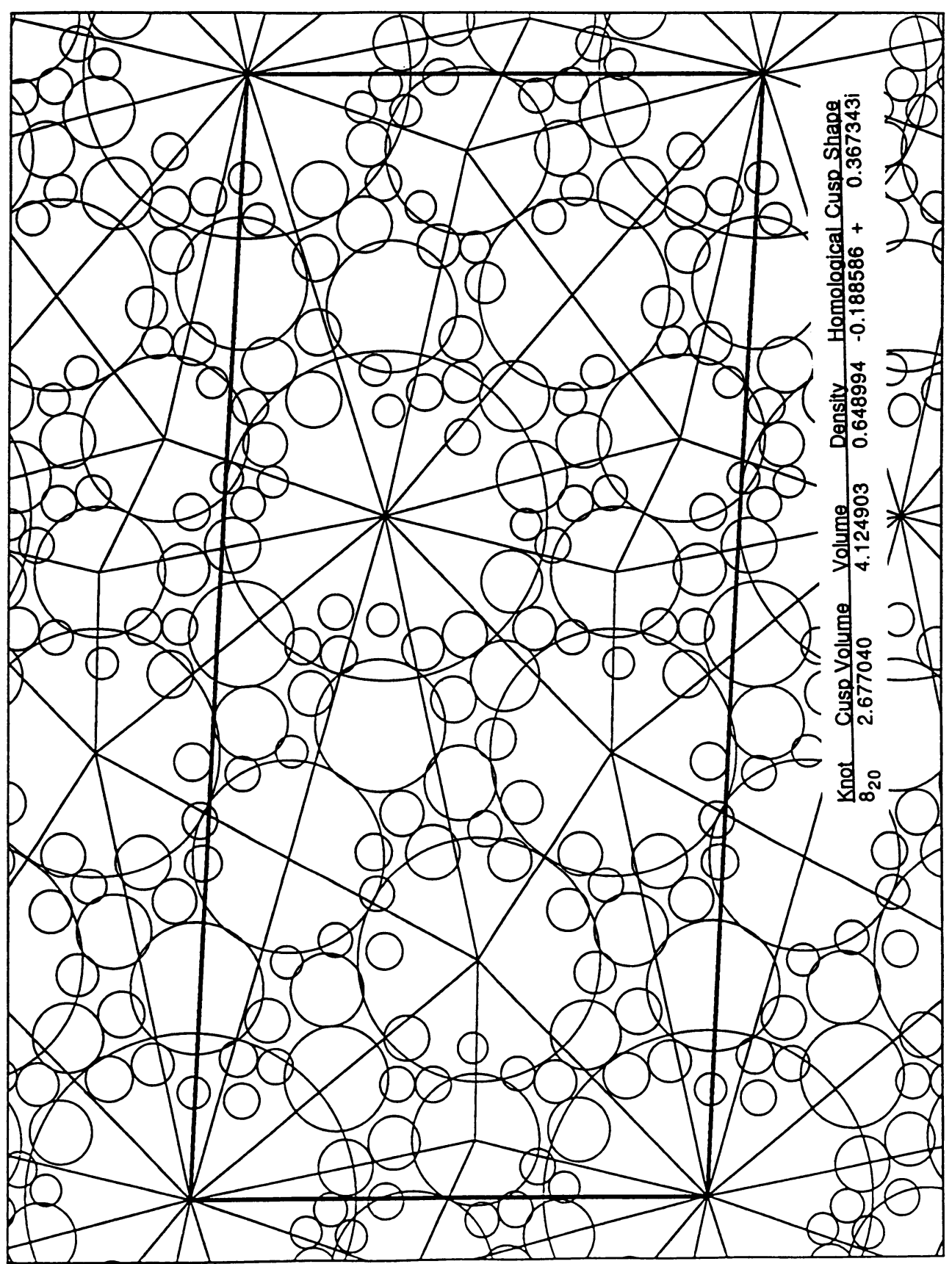

TABLE 2 (continued) 


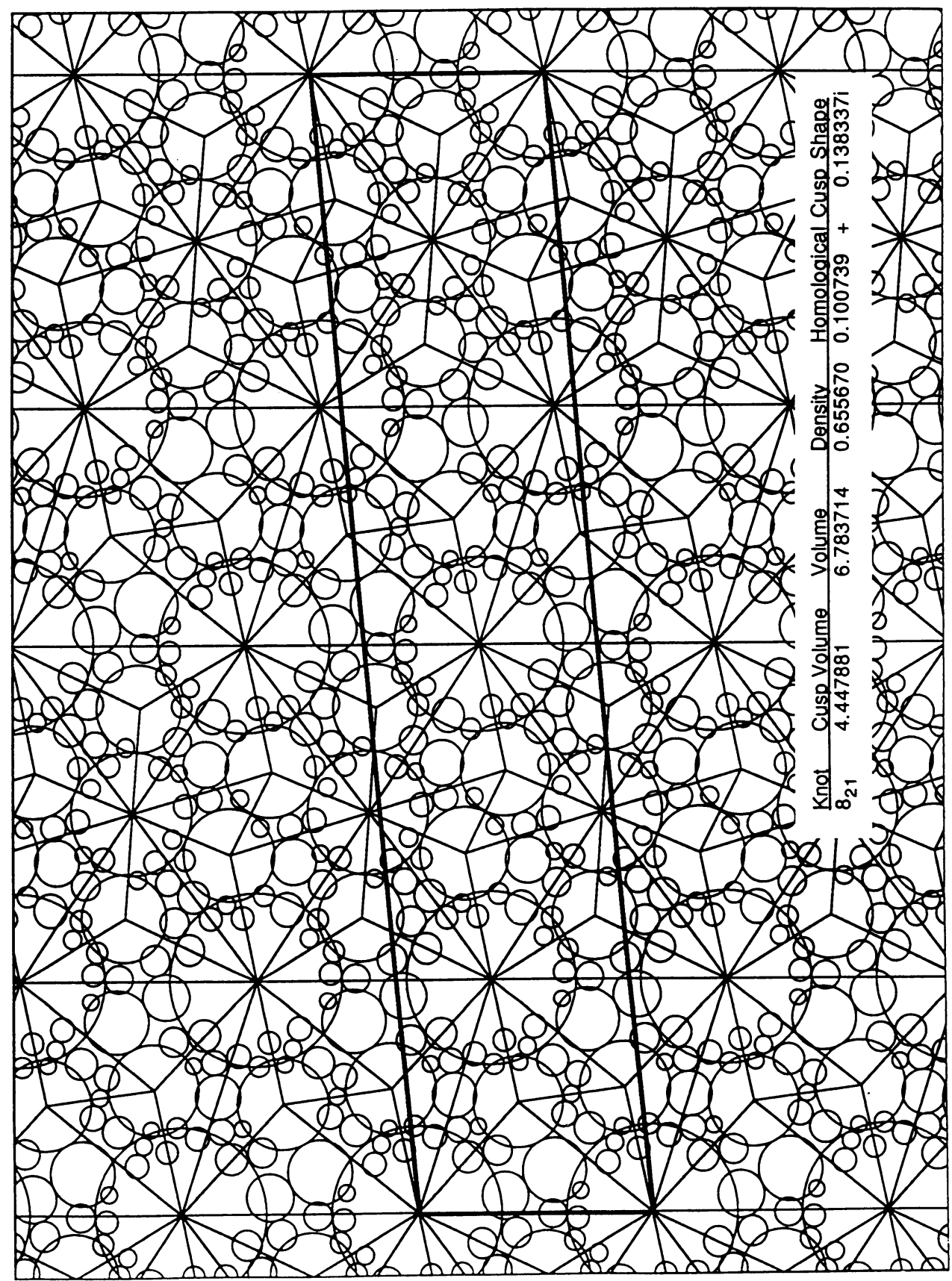

TABLE 2 (continued) 


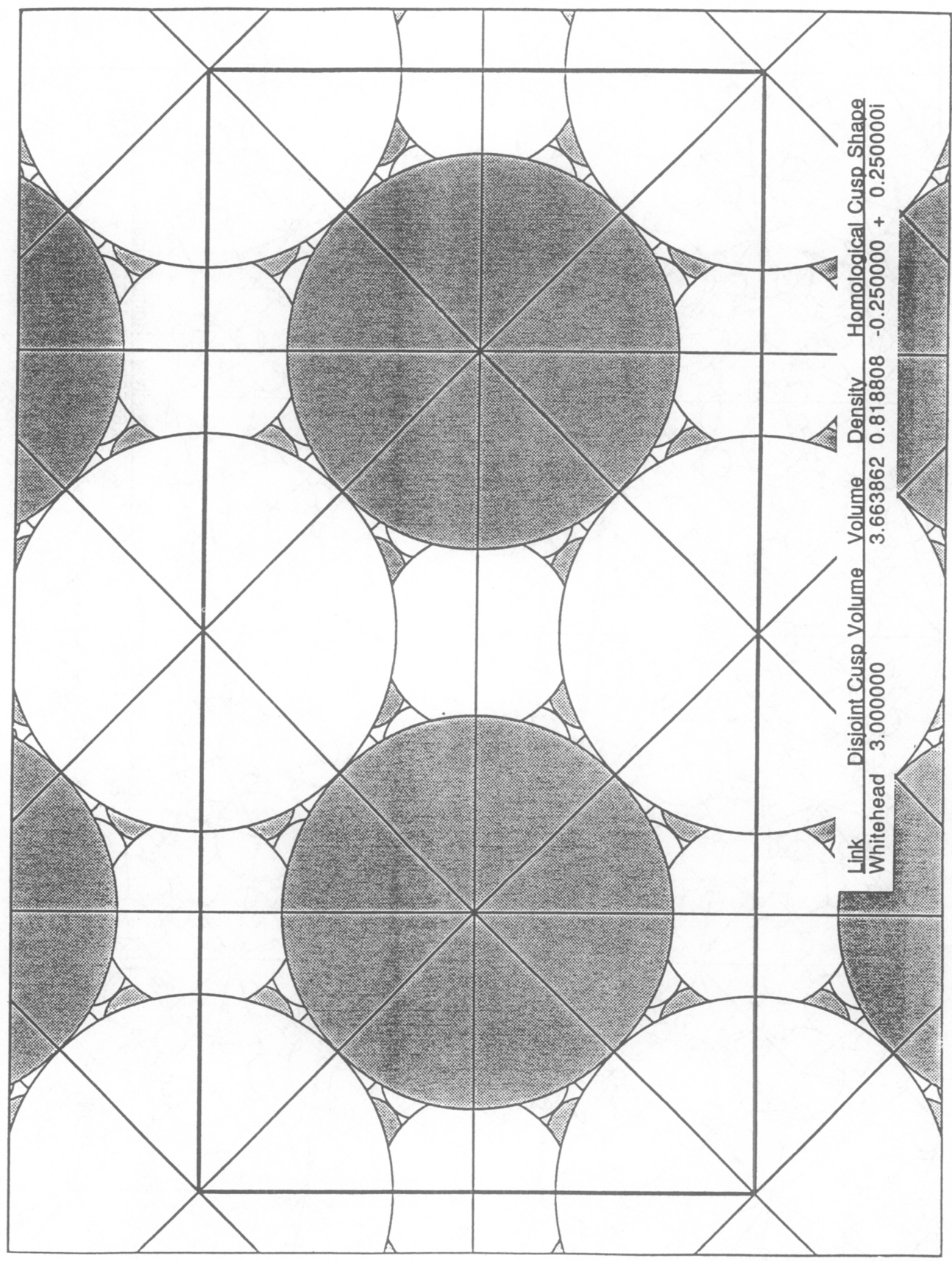

TABLE 2 (continued) 


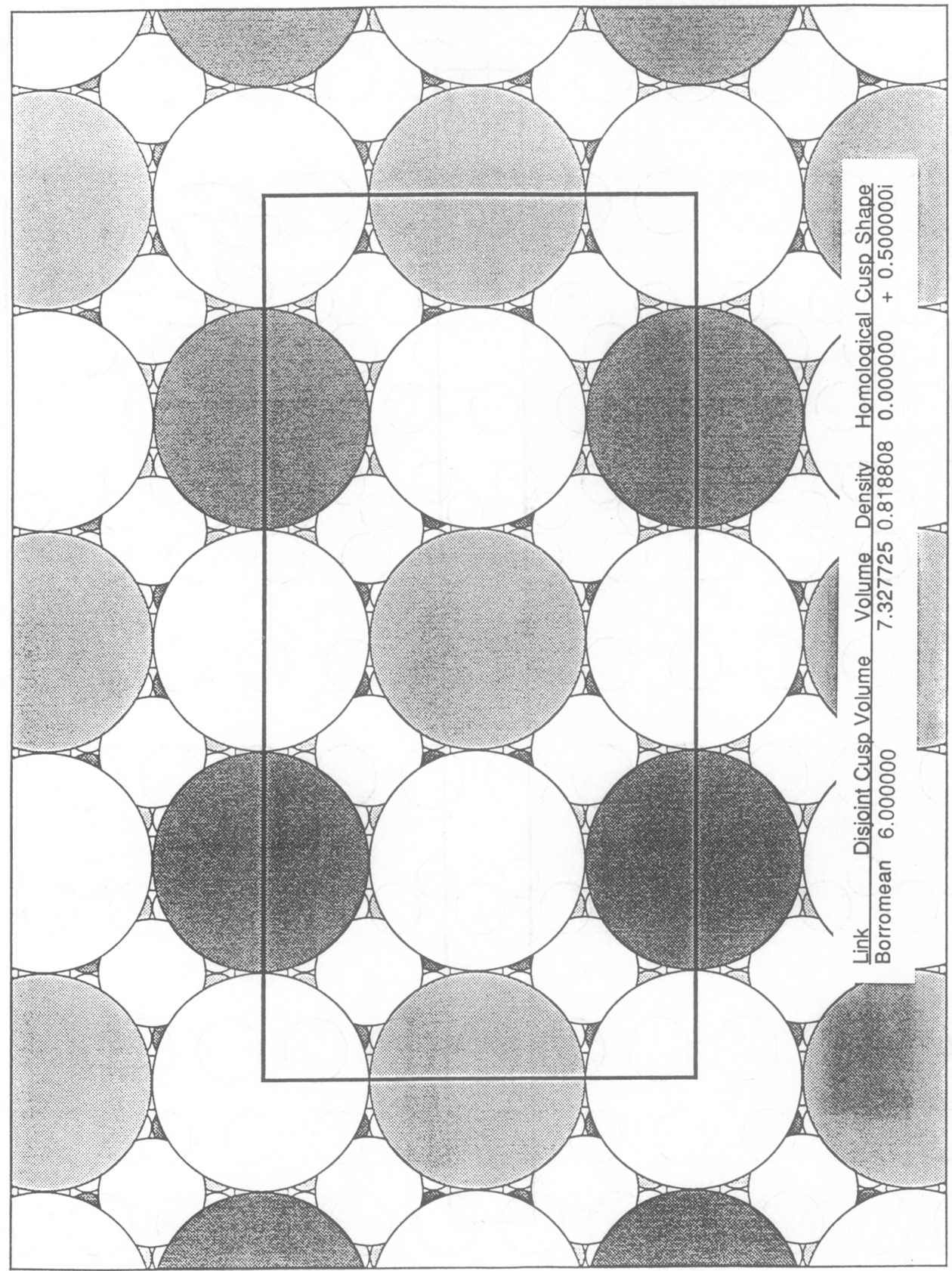

TABLE 2 (continued) 


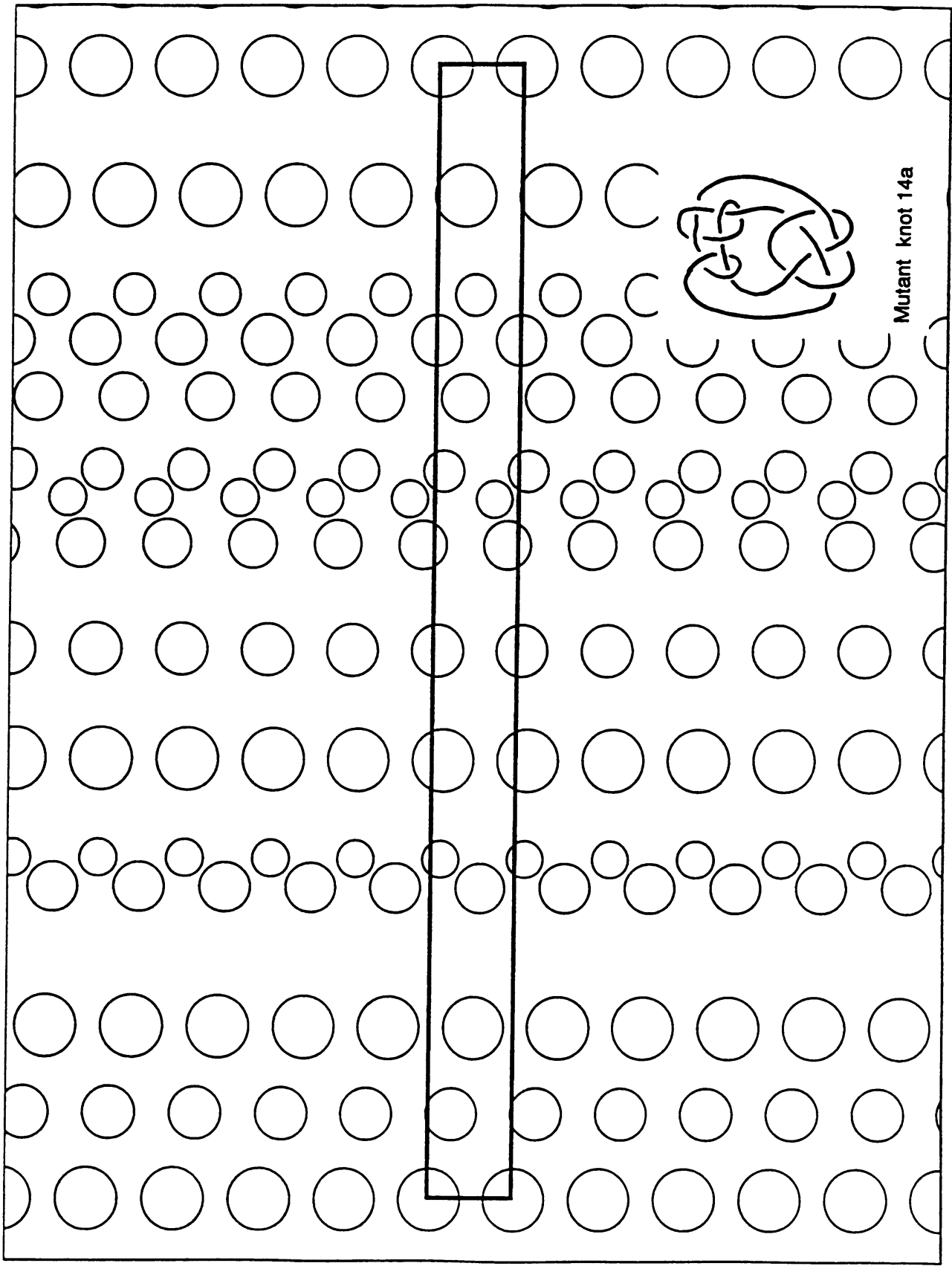

TABLE 2 (continued) 


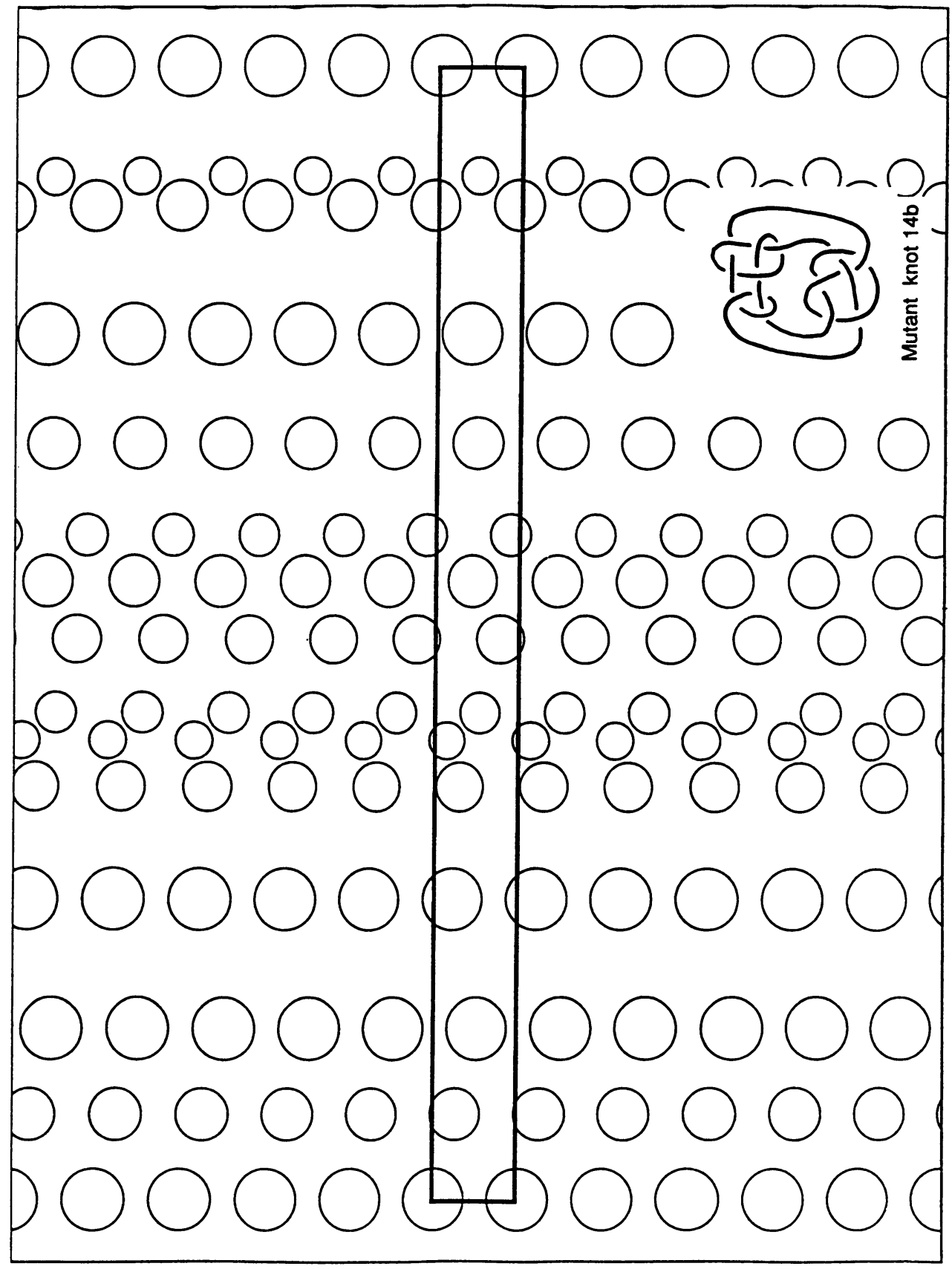

TABLE 2 (continued) 


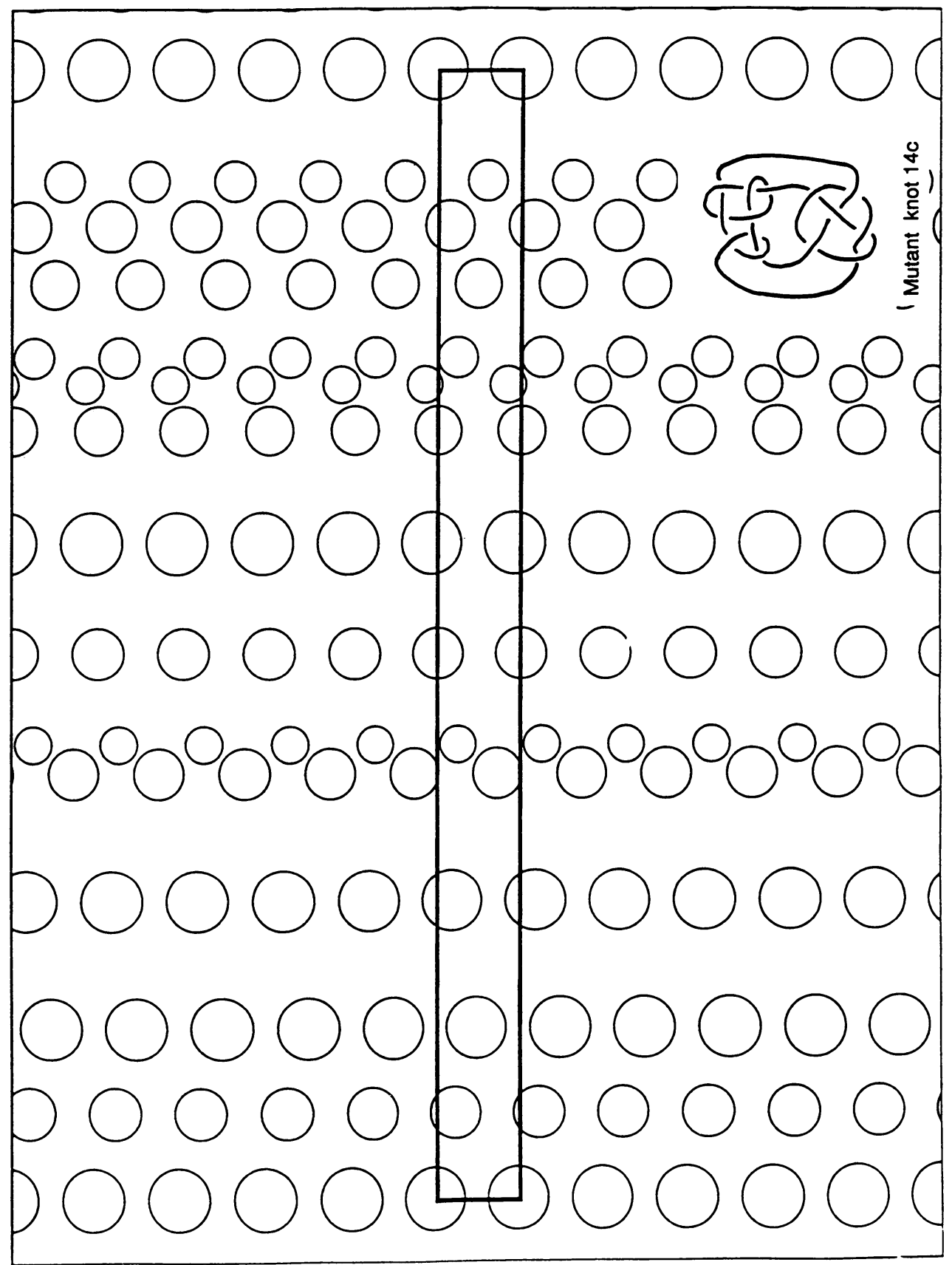

TABLE 2 (continued) 


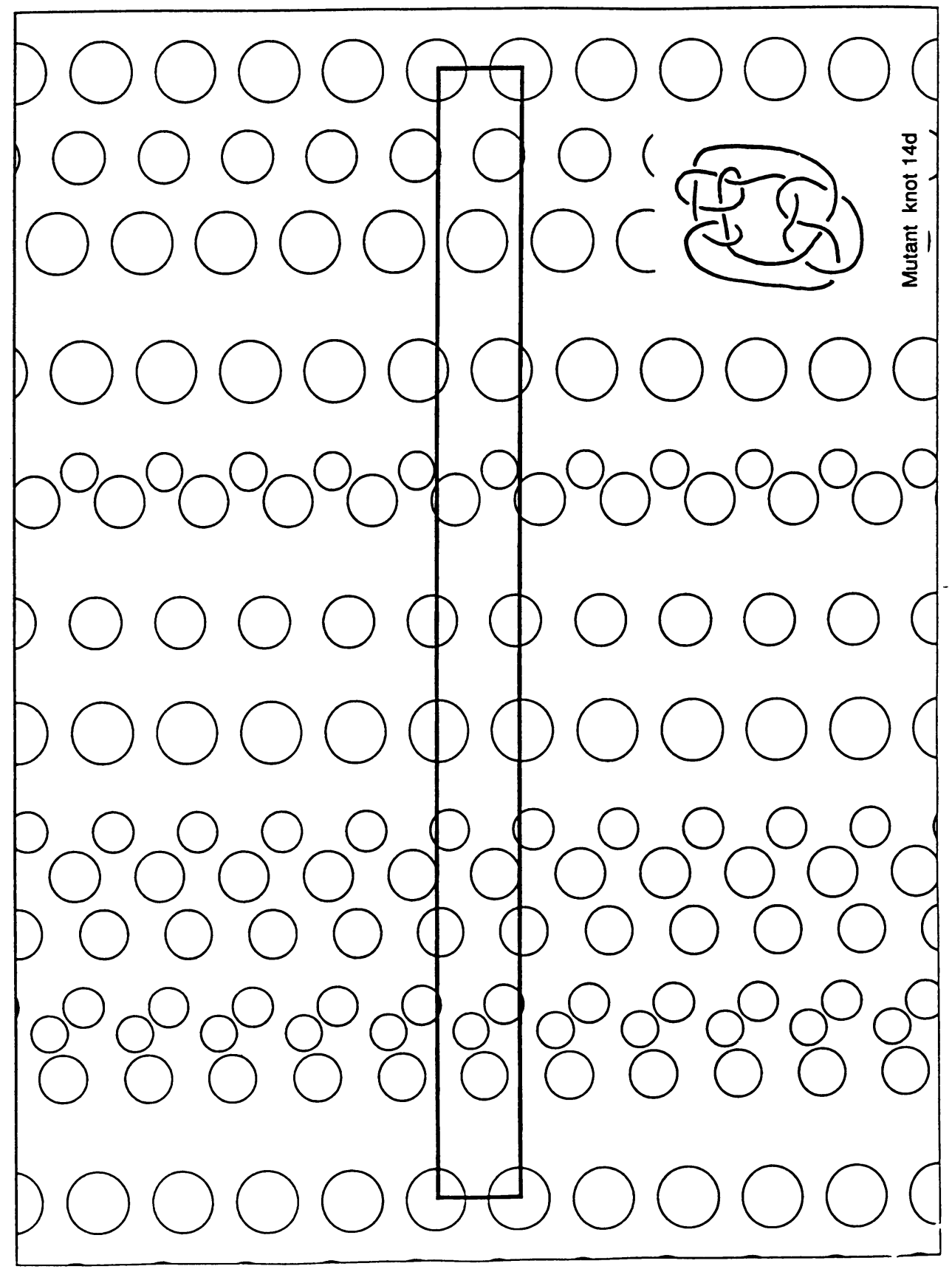

TABLE 2 (continued) 


\section{REFERENCES}

1. C. Adams, Thrice-punctured spheres in hyperbolic 3-manifolds, Trans. Amer. Math. Soc. 287 (1985), 645-656.

2. The noncompact hyperbolic 3-manifold of minimal volume, Proc. Amer. Math. Soc. 100 (1987), 601-606.

3. 555-565.

4. K. Boroczky, Packing of spheres in spaces of constant curvature, Acta Math. Acad. Sci. Hungar. 32 (1978), 243-326.

5. G. Burde and H. Zieschang, Knots, De Gruyter Studies in Mathematics, vol. 5, De Gruyter, Berlin, 1985.

6. L. R. Ford, Automorphic functions, McGraw-Hill, New York, 1929.

7. R. Meyerhoff, Sphere packing and volume in hyperbolic 3-space, Comment. Math. Helv. 61 (1986), 271-278.

8. R. Meyerhoff and D. Ruberman, The Chern-Simons invariant and mutation, J. Differential Geom. (to appear).

9. J. Milnor, Hyperbolic geometry: the first 150 years, Bull. Amer. Math. Soc. 6 (1982), 9-23.

10. R. Riley, An elliptical path from parabolic representations to hyperbolic structures, Lecture Notes in Math., vol. 722, Springer-Verlag, 1979, pp. 99-133.

11. D. Rolfsen, Knots and links, Publish or Perish, Berkeley, Calif., 1976.

12. D. Ruberman, Mutations and volumes of knots in $S^{3}$, Invent. Math. 90 (1987), 189-215.

13. W. Thurston, The geometry and topology of 3-manifolds, Lecture notes, Princeton University, 1978-79.

14. W. Thurston, Three-dimensional manifolds, Kleinian groups and hyperbolic geometry, Bull. Amer. Math. Soc. 6 (1982), 357-381.

15. J. Weeks, Hyperbolic structures on 3-manifolds, Ph. D. dissertation, Princeton University, 1985.

16. N. Wielenberg, Hyperbolic 3-manifolds which share a fundamental polyhedron, Riemann Surfaces and Related Topics: Proceedings of the 1978 Stony Brook Conference, Princeton Univ. Press, Princeton, N.J., 1981, pp. 505-513.

Department of Mathematics, Williams College, Williamstown, Massachusetts 01267

Department of Mathematics, University of Michigan, AnN Arbor, Michigan 48109

Department of Mathematics, Middlebury College, Middlebury, Vermont 05753 